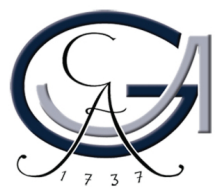

\title{
Lab-on-chip design to characterize pore-spanning lipid bilayers
}

\author{
Theresa Kaufeld \\ from Nienburg/Weser
}

Göttingen 2013

Dissertation

for the award of the degree

"Doctor rerum naturalium"

of the Georg-August-Universität Göttingen

within the doctoral program (Physics of Biological and Complex Systems) of the Georg-August University School of Science (GAUSS) 
Members of the thesis committee

Prof. Dr. Christoph F. Schmidt (Supervisor)

3rd Institute of Physics - Biophysics, Faculty of Physics,

Georg-August-University Göttingen

Prof. Dr. Claudia Steinem

Institute for Organic and Biomolecular Chemistry, Faculty of Chemistry

Georg-August-University Göttingen

Prof. Dr. Tim Salditt

Institute for X-Ray Physics, Faculty of Physics,

Members of the examination board

Prof. Dr. Christoph F. Schmidt (Reviewer)

3rd Institute of Physics - Biophysics, Faculty of Physics,

Georg-August-University Göttingen

Prof. Dr. Claudia Steinem (Reviewer)

Institute for Organic and Biomolecular Chemistry, Faculty of Chemistry

Georg-August-University Göttingen

Prof. Dr. Tim Salditt

Institute for X-Ray Physics, Faculty of Physics,

Georg-August-University Göttingen

Prof. Dr. Sarah Köster

Institute for X-Ray Physics, Faculty of Physics,

Georg-August-University Göttingen

Prof. Dr. Jörg Enderlein

3rd Institute of Physics - Biophysics, Faculty of Physics,

Georg-August-University Göttingen

Prof. Dr. Ulf Diederichsen

Institute for Organic and Biomolecular Chemistry, Faculty of Chemistry

Georg-August-University Göttingen

Date of oral examination: 23.10.2013 


\section{Affidavit}

I herewith declare that I have produced this dissertation without the prohibited assistance of third parties and without making use of aids other than those specified; notions taken over directly or indirectly from other sources have been identified as such. This dissertation has not previously been presented in identical or similar form to any other German or foreign examination board.

The thesis work was conducted from August 2009 to August 2013 under the supervision of Prof. Dr. Christoph F. Schmidt at the Third Institute of Physics Biophysics.

Göttingen, 28.08.2013 



\section{Contents}

1 Abstract 1

2 Introduction 3

2.1 The biological membrane . . . . . . . . . . . . . . 3

2.2 Methods for artificial membrane preparation . . . . . . . . 5

2.3 Lipid bilayers and lab on a chip . . . . . . . . . . . . . . 9

2.4 Mechanosensitive ion channels . . . . . . . . . . . . . . . . 11

3 Design and fabrication of a microporous substrate 15

3.1 Photolithography .................... 16

3.1 .1 SU-8 photoresist . . . . . . . . . . . . . 17

3.1 .2 SU-8 processing . . . . . . . . . . . . . . 18

3.1.3 Resolution and alternative methods of structure generation . 21

3.1.4 Process parameters for photolithographic fabrication steps . 22

3.2 Dry etch and deposition processes . . . . . . . . . . . . . . 23

3.2 .1 Reactive Ion Etch (RIE) . . . . . . . . . . . . . . 23

3.3 Deposition processes ....................... 25

3.3.1 Plasma Enhanced Chemical Vapor Deposition (PECVD) . . 25

3.3.2 Deposition of metals . . . . . . . . . . . . . 26

3.4 Silicon etching in alkaline solution . . . . . . . . . . . . 28

3.5 Design considerations . . . . . . . . . . . . . . 30

3.6 Fabrication scheme ..................... 33

4 Characterization of the substrate $\quad 39$

4.1 Determination of pore-radius and roundness . . . . . . . . . 40

4.2 Atomic Force Microscopy . . . . . . . . . . . . . . . . . . . . . . . . 41 
4.3 Electron microscopy _. . . . . . . . . . . . . . . . . 42

4.4 Results and discussion of AFM and SEM experiments . . . . . . . . 44

4.4.1 SU-8 coated substrates . . . . . . . . . . . . . . . 44

4.4 .2 Silicon dioxide coated substrates . . . . . . . . . . 46

4.4 .3 Silicon nitride substrates . . . . . . . . . . . . . 47

5 Setup and sample chamber $\quad 49$

5.1 Setup............................... 50

5.2 Sample chamber . . . . . . . . . . . . . . . . 53

5.3 Lipid bilayer formation . . . . . . . . . . . . . . 54

5.3.1 Preparation of Giant Unilamellar Vesicles (GUVs) . . . . . 54

5.3.2 Preparation of lipid bilayers and alamethicin reconstitution . 55

6 Impedance Spectroscopy $\quad 59$

6.1 Theoretical Background ................... 60

6.1.1 Equivalent circuit elements . . . . . . . . . . . 63

6.2 Results and discussion of impedance spectroscopy experiments . . . 68

6.2.1 Electical models for silicon/silicon nitride based porous substrates ........................ 69

6.2.2 Impedance spectra of the microsubstrates with integrated electrodes . . . . . . . . . . . . . 73

6.2.3 Impedance spectra of lipid bilayers on the microsubstrates . 76

6.2.4 Theoretical pore resistance . . . . . . . . . . 78

6.2.5 Capacitance from triangle voltage pulses . . . . . . . 80

6.2.6 Experimental procedure ............... 81

7 Alamethicin single-channel experiments 83

7.1 Voltage clamp single-channel recordings . . . . . . . . . . . . 83

7.1.1 Single-channel recording and analysis . . . . . . . . . 84

7.2 Alamethicin . . . . . . . . . . . . . . 85

7.2.1 Results and discussion of alamethicin single-channel experiments .................... 86 
8 Polycystin-2 $\quad 89$

8.1 Polycystin-2 expression . . . . . . . . . . . . . . . 92

8.1.1 Vector and restriction digest . . . . . . . . . . . . 92

8.1 .2 Dot blot ........................... 94

8.1.3 Cell-free expression of polycystin-2 . . . . . . . . 96

9 Conclusions \& Outlook 101

$\begin{array}{ll}\text { List of figures } & \text { XXI }\end{array}$

$\begin{array}{lll}\text { List of tables } & \text { XXVII }\end{array}$

$\begin{array}{ll}\text { Appendix } & \text { XXIX }\end{array}$ 



\section{CHAPTER 1}

\section{Abstract}

A powerful approach to study membrane proteins is the reconstitution in model membranes. Methods for artificial bilayer formation are e.g. membranes on a solid support, or the classical BLM. In this project, the focus was on the formation of lipid bilayers on porous substrates combining the stability of solid supports and the accessibility to both sides of the bilayer of the classical BLM which is necessary for electrical recordings of membrane channels. Commercially available porous substrates, however, are typically not suitable for low-noise electrical experiments or for a combination with further manipulation techniques. Therefore, a microporous substrate was designed and fabricated meeting several demands: (i) To perform multiple experiments on one chip, the substrate was devided into four arrays of pores with separate electrolyte compartments and electrical connections. (ii) A PMMA/PDMS sample chamber was designed in a way that allows the exchange of solutions throughout the experiment. (iii) An integrated electrode facilitates switching between different electrical measurement techniques and allows better access for microscope objectives.

The substrate was fabricated in a multi-step cleanroom technology based process. Porous arrays of 900 and 9 pores were etched into a thin silicon nitride layer, but only the small 9-pore arrays turned out to be suitable for lipid bilayer formation, because the vesicles rupturing on the pore array do not fuse completely and leave uncovered pores, as fluorescence microscopy images showed.

The surface of the substrate and the appearance of the pores was characterized using atomic force microscopy and scanning electron microscopy and showed a 
low surface roughness and ordered pores. In addition, the pore-diameter was determined by a pixel analysis of brightfield microscopy images and matches the nominal pore diameter closely.

The substrate and the influence of the integrated electrode as well as lipid bilayer formation was investigated using impedance spectroscopy. The circuit model for the substrate was shown to be a simple $R(R C)$ circuit, whereas the integrated electrode contributes with an additional $\mathrm{R} / \mathrm{CPE}$ element and reveals diffusive behavior. Whether external or integrated electrodes are used does influence the lipid bilayer spectrum which is dominated by the $G \Omega$ resistance of the membrane and the high capacitance of the thin silicon nitride layer of the substrate. The theoretical pore resistance for cylindrical pores was calculated and agrees well with the experimental results.

As a tests for the suitability of the substrate and the newly built setup for voltage clamp recordings, alamethicin ion channels were functionally reconstituted into the lipid bilayers.

Additionally, the expression of polycystin-2, a supposedly mechanosensitive membranespanning protein, was adapted from H. F. Canitello and modified to be established for future experiments.

In summary, a versatile microporous substrate was developed that is suitable for solvent-free lipid bilayer formation and functional reconstitution of ion channels. The substrate can be adapted for many different techniques, such as optical microscopy, impedance spectroscopy and voltage clamp recordings. Because it is custom-built, modifications in terms of pore-size and number as well as functionalization of the surface can be adjusted easily. 


\section{CHAPTER 2}

\section{Introduction}

\subsection{The biological membrane}

The first attempts to find models for cell membranes emerged in the 1930s. The lipid bilayer model of J. F. Danielli and H. Davson, who described the membrane as a layer of lipids on which protein films are absorbed, was one of the first of those models [1]. It was replaced by the fluid mosaic model proposed by S. J. Singer and G. L. Nicolson in the 1970s [2]. They describe the biological membrane as a two-dimensional fluid, in which globular, amphiphatic proteins are embedded (figure 2.1). The possible role of interactions between the lipid bilayer and proteins is already mentioned, but the membrane is rather considered to be a protection layer for the cell cytosol and a passive matrix that is needed for the proteins to function.

Lipids are amphiphilic molecules with a hydrophobic chain and a hydrophobic headgroup, which makes the assembly into a bilayer with a hydrophobic core and the hydrophilic headgroups facing the aqueous cytosol the thermodynamically preferred configuration. Membranes of cells and organelles consist of a wide variety of lipids. Gylcerophospholipids, that consist of sn-glycero-3-phosphate with fatty acid esters on $\mathrm{C} 2$ and $\mathrm{C} 3$, are the most abundant types of lipids and show various types of modifications i the headgroup (phosphatidylcholin (PC), phosphytidylethanolamine (PE), phosphatidyl-serine (PS), phospho-inositides) as well as in the fatty acid chains (chain length and degree of unsaturation). Sphingolipids (Sphingomyelin, cerebrosides and gangliosides), which share a ceramide as the base compound, and cholesterol, but other sterol components can also be found, are 
important components of biological membranes [3].

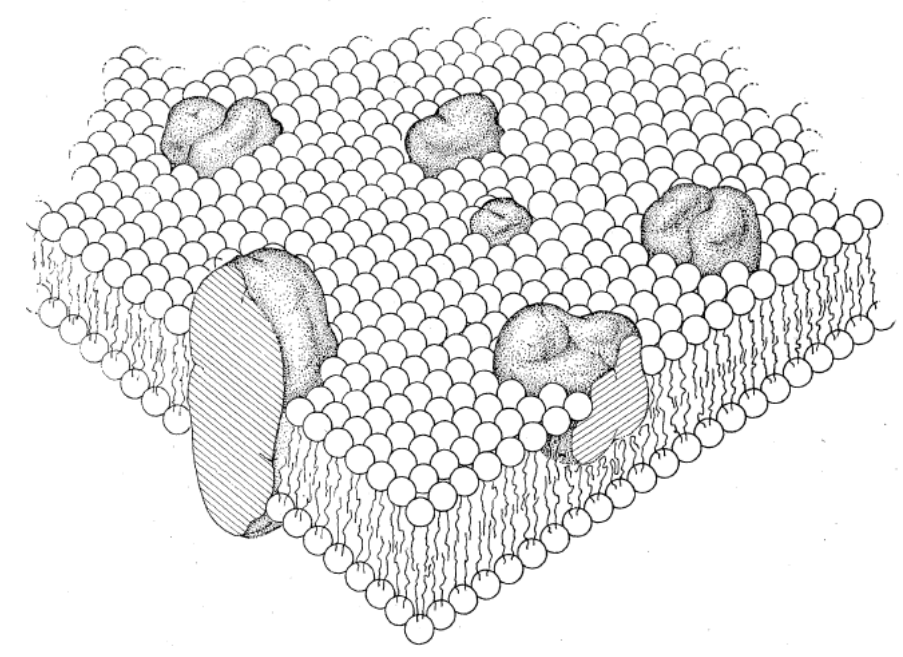

Figure 2.1: The fluid mosaic model as proposed by S. J. Singer and G. L. Nicolson, adapted from [2].

In eukaryotic cells, more than 1000 lipids can be distinguished [4]. Not only the lipid distribution in organelles differs, as it was shown for example for the organelles of TLR -4-activated macrophages [5], but even leaflets of lipid bilayers are asymmetrically composed; in the plasma membrane, for example phosphatidylcholines and sphingolipids are found mostly in the outer leaflet, whereas the cytosol-facing leaflet contains phosphatidyl-serine, phospho-inositides and phosphatidyl-ethanolamines [6].

This variety of different components led to the assumption that the cell membrane plays an active role in regulating protein assembly and function. The highly debated raft-hypothesis of K. Simon [7], for example, suggests that lipids form sphingomyelin- and cholesterol-enriched, highly dynamic liquid-ordered nanodomains of higher packing density than the surrounding liquid-disordered phase, which leads to membrane subcompartmentalization, and is thought to play a role in protein sorting, trafficking and endocytosis [8]. It is also known that the conductivity and open-probability of ion channels can be altered by embedding them in either anionic or neutral lipid bilayers or that they are even activated by stretching or bending of the membrane. This topic is regarded more closely in section 2.4 of this chapter. Research has become increasinly interested in the interaction 
of the cell membrane with the underlying cytoskeleton, as there are proteins that act as linker molecules between both (like myosin 1c [9]) or that are regulated by cytoskeletal components, as it was shown for the membrane channel protein polycystin-2 ([10], section 2.4).

In order to investigate all these complex processes, the interplay and purpose of the different components, artificial model systems that reduce this complexity to a few well-defined parameters are crucial.

\subsection{Methods for artificial membrane preparation}

Many methods for artificial lipid bilayer formation and ion channel reconstitution are nowadays available [11, 12]. Solid hydrophilic substrates like silicon, glass or mica are the most stable systems for membrane formation [13]. Lipid bilayers are mostly prepared by rupture and fusion of large (about $100 \mu \mathrm{m}$ ) or small (about $20 \mu \mathrm{m})$ unilamellar vesicles. Because of the solid support, they are quite robust and stable for days. This makes them very useful for more invasive methods, such as atomic force microscopy, which has been used to study domain formation [14]. Fluorescence microscopy can be applied in order to investigate protein assembly by monomer bleaching [15], binding studies of membrane associated proteins, such as actin polymerization, the pH-sensitivity of fluorescing molecules, or cell binding to a membrane-anchored receptor $[16,17,18]$. On top of that, temperature dependent effects [19] can be investigated and methods that require reliably planar surfaces such as X-ray spectroscopy commonly use solid supported lipid bilayers [20].

A drawback, however, is the interaction between incorporated proteins or the membrane itself and the supporting substrate. As a result, the diffusion of lipids and proteins is restricted, even in the upper leaflet, compared to the diffusion in lipid vesicles or free-standing membranes [21]. The diffusion of membrane-spanning proteins, that are much thicker than the membrane, can be completely inhibited.

Attempts to avoid this problem were made by modifying the supporting surface. Lipid bilayers were prepared on polymer cushions or spacer molecules creating a small space between the bilayer and the substrate [22]. Nevertheless, solid supports still deny access to the lower leaflet of the membrane, which is crucial for any electrophysiological lipid bilayer or ion channel recordings. 
Vesicles, especially Giant Unilamellar Vesicles (GUVs) with a low membrane curvature, are more natural mimics for cells. Here, no interactions of membrane-spanning proteins or lipids with a substrate alter the lipid mobility or the protein function and the vesicle shape resembles the shape of a cell. Naturally, they are very suitable model systems for studies of curvature effects because changes in their shape are not suppressed by tethering surfaces of any kind. For example, the effect of different lipid compositions or modified lipids [23, 24, 25] or of proteins which cause the formation of tubular structures or invaginations $[26,27]$ on the shape of the vesicles can be explored. The effect of the environment, such as the behavior of vesicles in $\mathrm{AC}$ or $\mathrm{DC}$ electric fields [28, 29], is also being investigated, providing a better insight into the reaction of living cells to electric fields or the reaction to a phase-separated inner solution [30].

While this methods has several benefits, its drawbacks regarding electrophysiological measurements lay in the round, closed shape of the vesicles, because it denies access to the inner leaflet of the vesicle and changing solutions inside the vesicle is impossible. Nevertheless, electrophysiological experiments in whole-cell configuration have also been performed [31].

The classic method used in the past decades for single channel recordings in artificial lipid bilayers is the preparation of Black Lipid Membranes (BLMs). In general, these membranes are prepared by applying lipids, dissolved in a non-polar solvent like n-decane on a small hydrophobic aperture (Mueller-Rudin technique [32]). The lipids reassemble at the water-solvent interface and the solvent diffuses into solution, so that only the bilayer remains in the center of the aperture with residual solvent at the edges of the aperture. The name stems from this preparation effect: When the lipid/solvent mixture is illuminated with white light, it shows the Newtonian color spectrum due to interference and reflection on the surface. The thickness decreases in the thinning process and the 4-6 $\mathrm{nm}$ thick lipid bilayer causes destructive interference, in reflection causing the lipid bilayer to appear black when observed through a microscope [33].

BLMs were the first artificial system that made electrophysiological experiments possible. Already in 1974, cytochrome oxidase, H-ATPase and bacteriorhodopsin were reconstituted into BLMs by the fusion of proteoliposomes [34]. Since then, BLMs have become the most widely used method for studying transport phenom- 
ena across the membrane.

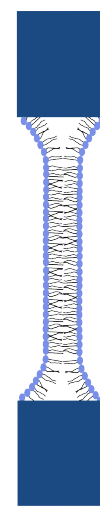

black lipid membrane (BLM)

biological membrane
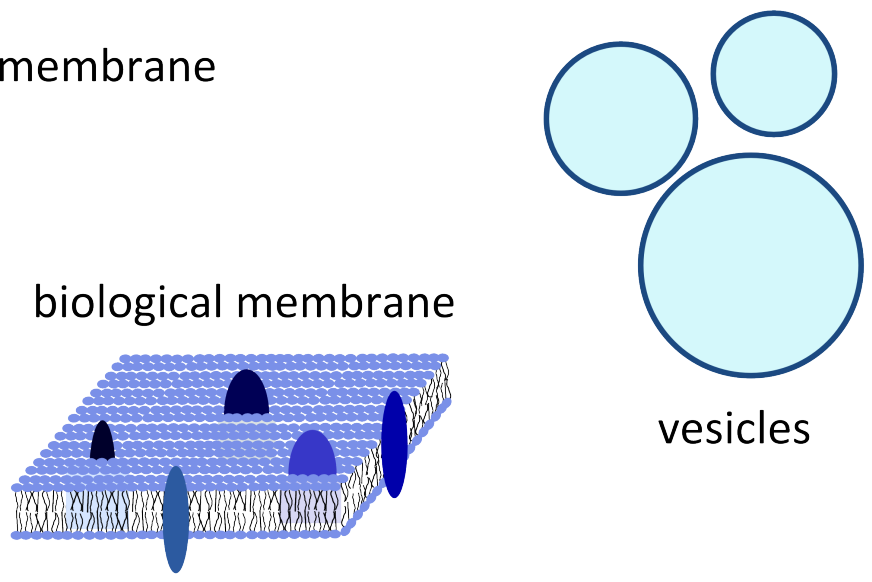

membranes on porous

substrates ( $\mu$-BLM)

solid supported membrane

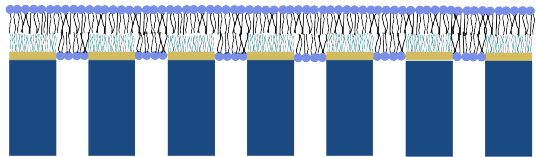

Figure 2.2: Different techniques for biological membrane models are (clockwise) the Black Lipid Membrane (BLM), Giant Unilamellar Vesicles (GUVs), solid supported membranes and lipid bilayer on porous supports (microBLM or nanoBLM).

A variant of the Mueller-Rudin preparation is the Montal-Mueller technique, where lipids at the air-water-interface of two buffer compartments, which are connected by a small aperture, are raised by increasing the volume of the buffer, so that the two monolayers meet, forming a bilayer over the aperture between the buffer compartments [35]. This kind of preparation allows the generation of an almost solvent-free lipid bilayer and the possibility of preparing them with asymmetric lipid compositions in each leaflet. T. Gutsmann and U. Seydel for example observed a different behavior of the outer membrane protein of gram-negative bacteria for symmetric and asymmetric lipid bilayers [36].

Substrates used for all variants of BLM preparation are hydrophobic polymers like Teflon or SU-8 [37], silanized silicon nitride [38] or silicon [39].

To allow as many proteins as possible to incorporate into the BLM, the apertures are quite large (in the range of several tens of microns to millimeters), which in 
turn diminishes the long term stability of the membranes in the apertures. To raise the amount of data that can be generated in one experiment, approaches toward screening processes have been made, e. g. by preparing BLMs in more than one aperture in one experiment [40]. A preparation related-problem, especially for BLMs, is the residual solvent remaining at the rims of the apertures which may alter membrane properties in terms of mechanical properties [41].

Langmuir-Blodgett/Langmuir-Schaeffer membranes are prepared from lipid monolayers at the air-water-interface by raising a substrate vertically from the water, thereby attaching the lipid monolayer with the hydrophilic headgroup facing the substrate to it. By lowering the substrate again, a second monolayer attaches to the first one, and the bilayer forms.

A technique which combines the stability of the solid supported membranes and the good electrophysiological properties of the BLMs is the formation of membranes on porous substrates. An advantage of these systems is the possibility of solvent-free membrane preparation, because the pores are usually small enough to form membranes by vesicle rupture. Also, both sides of the membrane are accessible. Most of these systems, especially those used for electric recordings, have pores with diameters in the range of tens to hundreds of nanometers. That is very advantageous for a good stability of the membrane and therefore a low leak current in electrophysiological experiments, which is a result of uncovered pores. It has been shown for painted pore-spanning lipid bilayers that the stability increases by a factor of 30 when the pore diameter is decreased from $800 \mathrm{~nm}$ to $200 \mathrm{~nm}$ [42]. For this reason, the focus was for a long time to fabricate substrates with extremely small pores using focused ion beam milling or high resolution photolithography techniques (chapter 3.1.3) to enhance the stability of the membranes. With pores that are only 50-200 $\mathrm{nm}$ in diameter, A. P. Quist et al. were able to image porespanning lipid bilayers and simultaneously record single-ion-channel currents from membrane-incorporated gramicidin [43]. Kresak et al. positioned and ruptured singled GUVs over nanopores or arrays of nanopores [44] with diameters in the range of 50-600 nm. Porous alumina substrates with pore-diameters of $60 \mathrm{~nm}$ that were functionalized with a cholesterol-spacer via thiol-gold-chemistry were used by E. K. Schmitt et al. for the investigation of lipid bilayers by impedance spectroscopy [45]. These large arrays are very suitable for lipid bilayer formation by 
the painting technique, but, as the preparation of solvent-free lipid bilayers on porous substrates always involves vesicle spreading, it is quite difficult to achieve a completely intact bilayer. For substrates with a small number of or even single nanopores, on the other hand, the probability of protein insertion is very low, making multi-channel experiments or the investigation of cooperative effects difficult. None of the nanoporous substrates with sub-100 nm diameters can be used for optical microscopy, because of the resolution limit of the microscopes. Therefore, substrates with larger pores have been used for optical microscopy techniques. For any studies of mechanical properties of the membrane, a large free-standing area is needed in order to probe the lipid bilayer without interfering with the pore rims. Thus, the focus turns to the development of techniques for the preparation of solvent-free lipid bilayers on larger pores [11, 46]. The limiting factor for the pore size is, apart from the lack of stability of membranes on larger pores, the size of vesicles that can be generated, as it turned out to be difficult to fuse GUVs on porous substrates to form an electrically insulating lipid bilayer.

\subsection{Lipid bilayers and lab on a chip}

Many attempts have been made to generate lipid bilayer systems that can be prepared solvent-free, are suitable for screening applications, and accessible for both optical and electrical measurement techniques.

Biosensors that are used for medical or pharmaceutical application such as the screening of biomolecules (e.g. screening for enzyme inhibitors) require the reliable production of either homogeneous arrays of a target enzyme or heterogeneous arrays for investigating the effect of different samples (e.g. blood, saliva or urea) on one protein or antibody [47]. Lab on a chip systems for lipid bilayers offer the performance of experiments in geometrically confined, small spaces that minimize sample volumes and are therefore not only interesting from a basic research point of view.

Such geometrically defined lipid bilayer arrays can be liposomes that are attached to functionalized solid supports (e.g. by covalent bonding of biotin/streptavidin or DNA linkage) [47]. Printing or spotting methods are an emerging tool for creating solid supported lipid bilayers. In principle, all of these methods use lipids as 
an ink that is applied either via direct writing or on pre-patterned substrates. In micro-contact printing, lipid solutions are applied to a patterned stamp which is then used to print the lipids on more adhesive substrates [48]. A method that reverses this approach is micro-contact displacement, where lipids are removed from a continuous lipid bilayer [49]. Dip-pen nanolithography is a direct writing method, where lipids are applied to a surface by using an AFM tip or a micro-pipette as a nozzle [50]. In inkjetprinting, lipid vesicles are applied to a pre-patterned substrate to rupture in defined regions [51]. Non-contact printing methods apply very small volumes of highly-concentrated vesicle solutions on hydrophilic substrates. The spot size is limited by the subsequent rinsing step, which terminates the diffusion of unruptured vesicles [52].

Microfluidis are used for the formation of droplet interface bilayers (DIBs), which form from vesicle-containing aqueous droplets in oil. The vesicles inside the droplet form a monolayer at the oil-water interface and by bringing two (or more) vesicles into close contact, the oil is excluded and a lipid bilayer, or even lipid bilayer networks, forms. With these systems, it is possible to directly form asymmetric lipid bilayers. Since a large number of these lipid bilayers can be formed and the formation is very reproducible, they are very promising systems for drug screening and high-throughput experiments [53].

Such printing and microfluidic techniques are highly reproducible and suitable for automation and are valuable techniques for binding studies. However, especially the contact-printing methods are also restricted to the robust solid supported membranes and are not applicable to the fragile free-standing membranes.

Progress has also been made towards free-standing lipid bilayer arrays for screening applications [54]. H. Susuki et al. have developed a screening device in which BLMs in porous parylene sheets are formed that are incorporated in a 96-well plate. All 96 wells are electrically individually addressable, which makes high throughput screening of many bilayers at a time possible [40]. P. V. Ganesan and S. G. Boxer developed a membrane interferometer, that detects ion channel gating by the change in the curvature induced by the osmotic pressure that builds when sodium ions are translocated through gramicidin channels reconstituted in lipid bilayers spanning arrays of micro-wells [55].

Another aspect of the possibilities that come with micro- and nanolithography methods are the development of substrates that are designed in a specific way for 
reconstitution and manipulation of either proteins or membranes. These systems and substrates are needed for a better understanding of specific aspects of the interaction between lipid bilayers, proteins and the cytoskeleton.

Substrates that are not only the supporting material for the lipid bilayer, but also form the source for bilayer manipulation, are, for example, piezoelectric substrates (quartz glass) that have been used to directly mechanically stimulate the lipid bilayer on top of them and simultaneously also the electric response of incorporated ion channels [56]. F. Yi et al. used nanofibres, spanning a hole in a PTFE substrate for mimicking the cell cytoskeleton and were able to characterize phospholipid membranes reconstituted on these substrates [57].

\subsection{Mechanosensitive ion channels}

One future application for the microporous substrate that has been designed and characterized in this work is the investigation of mechanosensitive ion channels (MSCs), which open in response to forces, transduced by the lipid bilayer they are embedded in.

Although biosensors are widely used by now for electrophysiological experiments and have proven to be valuable tools for characterizing voltage-gated or ligandgated ion channels, one class of ion channels, mechanosensitive ion channels, is still not easily accessible. In this section, a brief overview of mechanosensitive ion channels and the approaches of investigating their properties, such as the forces they react to, will be given.

Mechanosensitive ion channels can be found both in prokaryotic and in eukaryotic cells. Prokaryotic MSCs include the well-studied families of MscL (especially the MscL from E. coli and Mycobacterium tuberculosis [58]), MscS and MscM (named according to their conductance Large, Small and Mini), and the archaea channels MscMJ and MscMJLR from M. jannashii [59]. In eukaryotic cells, the variety of MSCs is much larger than in prokaryotes. Examples of eukaryotic MSCs are the two-pore domain potassium channels $\left(\mathrm{K}_{2} p\right)$ that respond to physical an chemical stimuli, the DEG/ENaC family, which is selective for sodium-ions, is also gated by pH-changes (the family includes acid-sensing ion channels) and the tetrapeptide FMRFamide [60, 61]. TRP (transient receptor potential) ion channels, nonselec- 
tive $\mathrm{Ca}^{2+}$ permeable channels with six transmembrane domains, were found to be mechanosensitive and are considered to play a role in flow sensing in the primary cilia of epithelia cells $[62,63,64,65]$. Furthermore, simple channel-forming peptides like alamethicin or gramicidin, which are also used in this work for their voltage-dependence, react to mechanical stimuli $[66,67]$. Apart from these examples that were already studied in electrophysiological experiments, a large number of eukaryotic ion channels has yet to be discovered and analyzed.

Stresses that act on cells and induce channel opening can be separated into five classes according to S. Sukharev and F. Sachs [68]: osmotic stresses due to changes in the environment, muscle activity or actomyosin induced stresses leading to cell deformation, compressive or tensile stresses, flow induced shear stresses acting for example on primary cilia, and distortional stresses like in stereocilia of auditory haircells.

Two models of force transduction are discussed in the field. The first model proposes that the in-plane tension in the lipid bilayer gates the MSC. MscL and MscS, prokaryotic ion channels that act as pressure valves, have been reconstituted in artificial lipid bilayers and stimulated by hydrostatic pressure. Since no interaction with other components such as the cytoskeleton or other membrane proteins is possible, this model seems to be suitable at least for the mechanosensitive bacterial channels. For the eukaryotic MSC families $\mathrm{K}_{2 p}$ and MscS-like channels in plants this model seems applicable, too, because they open upon the same treatment as the prokaryotic channels MscL and MscS [61].

The second model proposes a direct connection of the MSCs to the cytoskeleton or extracellular matrix proteins that displace the channel gate. This tethered model may be suitable for some eukaryotic MSCs, including the TRP channel polycystin2 , for which several binding partners have been found that could connect it to the cytoskeleton (e.g. $\alpha$ actinin, kinesin-2 [10, 69, 70]). Also for several other eukaryotic MSCs proteins that would associate them to either the extracellular matrix or cytoskeleton are known and it seems likely that this association is somehow function-related.

In electrophysiological experiments of cells, MSCs were stimulated with suction of a patch pipette [71, 72], or, in case of whole-cell experiments, with shear, induced 
by a flow of the extracellular solution [73, 74, 75]. Only very few studies of reconstituted ion channels in artificial membranes exist, for example the MscL has been investigated in BLMs and activated by hydrostatic pressure [76, 77] and in an approach already mentioned in section 2.3 a piezo-sensitive substrate was used to increase the pore-size and thereby stretching the lipid bilayer on top of it [56]. However, for a better understanding of the forces needed to open the channels, for the specific effect of binding partners and the discrimination between different types of stimuli mentioned above (e.g. shear, stretch or the role of curvature change during bending), in vitro approaches, and in particularly lab on a chip approaches, are crucial. In such systems, stresses could be induced by more accurate probes like AFM-tips or optically trapped beads. This requires versatile substrates that offer the possibility of the combination of the stimulation techniques with electrophysiological experiments as well as free-standing lipid bilayers spanning pores that are large enough to rule out the interference of the probes with the substrate. 



\section{CHAPTER 3}

\section{Design and fabrication of a microporous substrate}

In this work, we designed and fabricated a microporous substrate for the formation of pore-spanning lipid bilayers and the reconstitution of ion channels. It was fabricated in a multi-step cleanroom technology based process. Figure 3.1 shows the microporous substrate that was designed and fabricated.

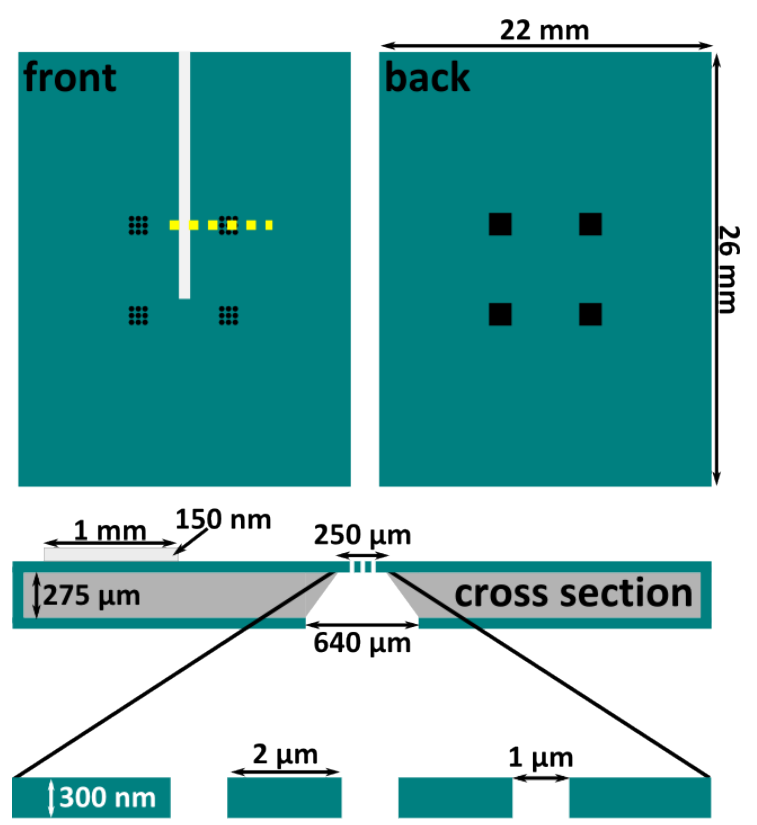

Figure 3.1: The microporous substrate designed and fabricated in this work. Details on the fabrication process are given in the text.

In this chapter, the methods used for the fabrication process are presented, the design considerations are explained and the fabrication process is described and 
discussed in detail.

\subsection{Photolithography}

Photolithography, also called Optical Lithography, is a widely used technique to create micrometer-sized structures on flat surfaces. In general, UV-sensitive polymers (photoresists) are exposed to UV-light through a photomask containing the desired structures which protects parts of the photoresist from the light. In case of positive tone photoresists, the crosslinked polymer is cracked and can be removed from the exposed areas, whereas the resist on the protected areas remains. When a negative tone photoresist is used, the UV-light initiates a crosslinking reaction of the unpolymerized material so that the exposed areas remain on the substrate (figure 3.2).

The structured photoresist can then act as a mask itself, for example in etching or deposition processes in MEMS applications (Microelectromechanical Systems) [78], but the photoresist can also be part of permanent structures, such as microchannels in microfluidic devices or as molds for soft-lithography processes [79]. The process parameters depend on the photopolymer characteristics, so the following chapter will only describe the processing of the SU-8 polymer, which was used exclusively in this work. 


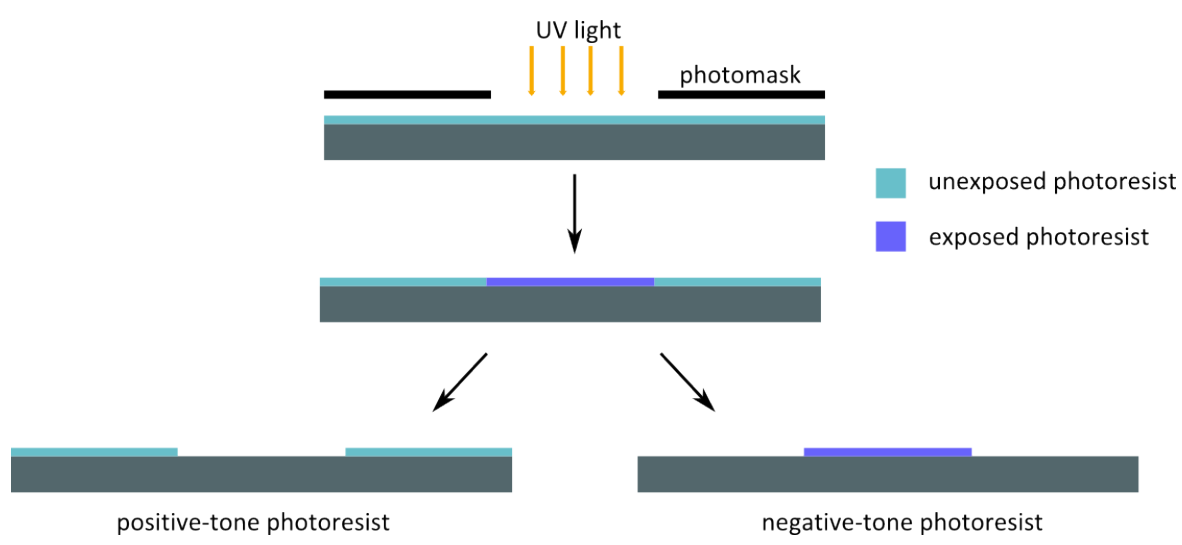

Figure 3.2: In photolithographic processes a photopolymer is exposed to UV-light through a mask defining the desired structures. The exposed areas are removed after exposure when positive tone-photoresist is used, whereas negative-tone photoresists remains on the substrate.

\subsubsection{SU-8 photoresist}

SU-8 is a negative tone photoresist that is mainly used as a permanent part of structures in microfluidic applications because of its biocompatibility, its stability against most solvents, acids and bases and the possibility of creating high-resolution high-aspect-ratio structures [80]. These properties are also advantageous whenever a robust photoresist, for example for long dry etch processes, is required. In this work, the SU-8 photoresist was used as a mask for the two dry etch steps defining the four pore areas in the silicon nitride before the $\mathrm{KOH}$ etching step (figure 3.10) and the structuring of the pores in the silicon nitride membrane (figure 3.11). Like all negative tone photoresists, SU-8 crosslinks after exposure to UV-radiation, which generates an acid catalyst that catalyzes the cationic polymerization of the photoresist [81, 82]. Each SU-8 monomer contains eight epoxy groups which are available for the crosslinking reaction (figure 3.3) [79]. 


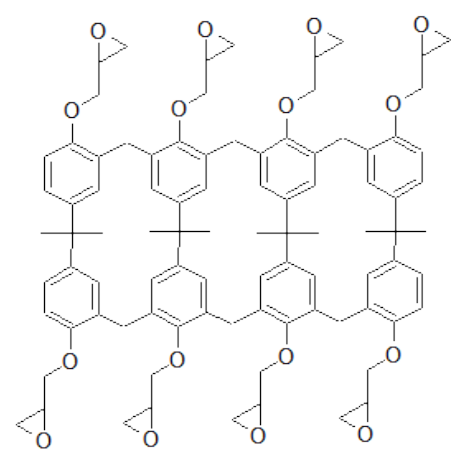

Figure 3.3: The SU-8 monomer contains eight epoxy groups which react during the crosslinking process.

\subsubsection{SU-8 processing}

Figure 3.4 gives an overview of the general procedure of SU-8 processing which consists of five steps: the coating of the phororesist on the substrate, the Soft Bake, the exposure to UV-light during which the photocatalyst is generated, the Post Exposure Bake (PEB), which accelerates the polymerization, and the development, where the uncrosslinked resist is removed.

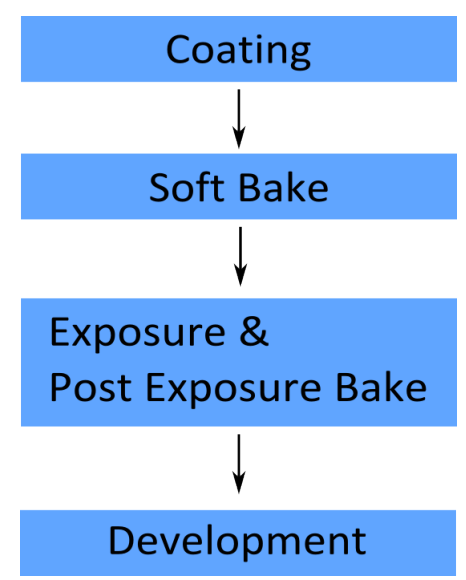

Figure 3.4: Overview of the SU-8 processing steps. 


\section{Coating}

The first step is the coating of the photoresist onto the substrate. There are several ways of resist deposition, the most commonly used method that was used also in this work, being spin coating, where an excess amount of resist is applied onto the substrate which is then rotated at high speed. This procedure results in a uniformly distributed resist layer whose thickness depends on the viscosity of the resist itself and the spinspeed. Other techniques are for example spray-coating or dip-coating [83].

\section{Soft bake}

After the coating of the photoresist, the substrate is heated to remove the solvent (cyclopentanone or $\gamma$-butyrolacton) by evaporation so that only the uncrosslinked photoresist and the photoinitiator (triarylsulfonium/hexafluoroantimonate salt) remain on the substrate. The soft bake is carried out to improve the adhesion between substrate and photoresist, to avoid sticking of the resist to the photomask during exposure and to avoid dark erosion during development, which are also problems occurring at too low temperatures or too short soft bake duration. On the other hand, if the temperature is too high or if the baking time is too long, thermal crosslinking of the photoresist can occur which results in a low development rate or makes through-development impossible with thicker photoresist layers. The optimal temperature and duration of the soft bake depend on the viscosity and the type of the photoresist used and guidelines are provided by the manufacturer of the particular photoresist [83, 84].

\section{Exposure and post exposure bake}

During the exposure, the photoinitiator is activated [81]. The photoinitiator is a triarylsulfonium/hexafluoroantimonate salt which releases the photoacid catalyst upon UV exposure [81, 82]. The photoacid catalyzes the SU-8 crosslinking reaction which is accelerated by the subsequent baking step (Post Exposure Bake, PEB). The photoresist is exposed to near UV-radiation $(365 \mathrm{~nm})$ through a photomask defining the desired pattern by protecting those parts of the photoresist which are supposed to be removed from the substrate during development. The 
SU-8 itself is mostly transparent for radiation of wavelengths above $350 \mathrm{~nm}$ so that even structures as high as $2 \mathrm{~mm}$ can be achieved in a very good quality. Which type of photomask is suitable for a certain process, depends mainly on the size of the structures. For patterning larger structures, an emulsion film mask can be used which gives good resolutions down to $5 \mu \mathrm{m}$ according to the supplier (JDPhoto, Oldham, UK), whereas for smaller structures chromium-glass masks are used, which are more expensive than emulsion film masks, but have a minimum resolution of about $0.5 \mu \mathrm{m}$ (ML\&C, Jena, GE).

Apart from choosing the optimal photomask for the process, the optimal exposure dose also influences the quality of the structures. Especially for thick SU-8 layers, too low exposure doses increase the dark erosion, meaning the development of under-exposed photoresist. This is due to the fact that the exposure of the resist occurs gradually from the top to the bottom of the layer. Therefore, in case of a too low exposure dose (too short exposure time or too low intensity), not enough photoacid is produced at the lower part of the layer leading to incomplete crosslinking during the PEB. Too high exposure doses, on the other hand, lead to crosslinking of parts of the structure which were supposed to be developed. This is due to scattering and diffraction of the UV radiation that increase with increasing intensity [85, 83]. As the optimal exposure dose is individual for each process (it depends on the substrate, the resist thickness and the light source) the determination of the optimal exposure dose requires a series of exposure doses for every process based on the exposure values recommended by the manufacturer.

\section{Development}

The uncrosslinked photoresist is removed by immersing the substrate into the developer (propylene glycol monomethyl ether acetate, PGMEA). The best results, especially for the small pore structures, were obtained by downward development, described by C.-M. Cheng and R.-H. Chen [86]. The uncrosslinked resist dissolves in PGMEA and produces a gel at the bottom of the substrate which is more and more diluted and eventually removed completely. Therefore developing the photoresist with the substrate upside down while agitating the developer, makes this process more effective and as a result the sidewalls of the structures turn out more vertical and no residual resist remains inside the pores. 
After the development, the substrate was heated to $180^{\circ} \mathrm{C}$ (Hard Bake) to improve the stability for the etching process.

\subsubsection{Resolution and alternative methods of structure generation}

The first type of photolithography that was developed and which is also used in this work is contact printing, in which photomask and substrate are brought into close contact before exposure. Its resolution is mainly limited by the quality and type of the photomasks used (emulsion film masks or chromium-glass masks, as explained above), the quality of the substrate and of the fabrication process itself. The quality of the fabrication process (e.g. sidewalls at the edge of the substrates and particles in the resist film) can cause gaps between substrate and photomask so that areas which are supposed be protected from the light are exposed due to diffraction inside the resist layer [87]. In fact, the resolution in contact printing photolithography is highly dependent on the tightness of the contact between mask and substrate. For resolutions around $1 \mu \mathrm{m}$ it is necessary to increase the contact by applying negative pressure between mask and substrate and additionally push the substrate towards the mask. These leads to an increase of the resolution, but also possible damage and contamination of the substrate and the mask.

Higher resolutions (below $1 \mu \mathrm{m}$ ) can be achieved by methods like projection printing, immersion lithography or the use of phase shift masks. Projection printing uses lenses between photomask and substrate which focus the mask pattern onto the substrate so that the resolution is increased without contact. Here, the resolution is limited by the wavelength of the radiaton source $(248 \mathrm{~nm}$ and $193 \mathrm{~nm}$ respectively for $\mathrm{KrF}$ and ArF eximer lasers, $13 \mathrm{~nm}$ for Extreme Ultraviolet Lithography [88]), and the numerical aperture of the focusing optics, which can be increased by using immersion fluids between the substrate and the focusing lens. With these improvements, a resolution of $45 \mathrm{~nm}$ can be achieved [89]. At such small resolutions the requirements for resists and masks are more challenging than for larger structures. For each new resolution photoresists have to be developed that are not only non-absorbent for the specific wavelength, but also very sensitive in their reaction upon exposure, because the resolution can even be limited by the diffusion of pho- 
tocatalyst molecules inside the resist layer. In the design process of photomasks not only the structures themselves have to be designed, but also the distortion of the pattern by diffraction effects (called proximity effects) have to be taken into consideration and are corrected by adding structures on the mask that cancel these effects. The prediction of these effects is, however, very complex and requires long computation times, especially if the pattern itself contains complicated structures. Electron- and ion-beam lithography circumvent the problem of finding appropriate photomasks, as those technologies work maskless. A focused beam of ions or electrons writes the pattern, which is generated only virtually in a CAD-based computer software, directly into the photoresist. The resolution is only restricted by the width of the beam and scattering effects on the surface of the resist [88]. Although this seems a very convenient method for generating high resolution structures, it also has drawbacks, because the processing time is very long (the process requires very low pressure) and the throughput is low because of the limited space in the electron beam chambers. Additionally, the method is only applicable for thin layers of photoresist (mostly PMMA-based) which do not withstand long etching processes. For that reason, even though the possibility of e-beam lithography is available in the cleanroom facilities and has been tried as an alternative to contact printing, this method was not further taken into consideration for this work.

\subsubsection{Process parameters for photolithographic fabrication steps}

Photolithographic processes are used in two of the fabrication steps of the microporous substrate: for patterning the pore areas and for patterning the pores themselves. In both steps SU-8 2001 (Michrochem, Newton, US) was used and the process parameters can be found in table 3.1. 
Table 3.1: Process parameters for photolithography fabricaiton steps

\begin{tabular}{|l|l|l|}
\hline Parameter & $\begin{array}{l}\text { Pore area patterning } \\
\text { (section 3.10) }\end{array}$ & $\begin{array}{l}\text { Pore patterning (section } \\
3.11)\end{array}$ \\
\hline \hline Coat & $3000 \mathrm{rpm}, \mathrm{ramp} 100 / \mathrm{s}$ & $3000 \mathrm{rpm}, \mathrm{ramp} 100 / \mathrm{s}$ \\
\hline Soft bake & $1 \mathrm{~min}, 95^{\circ} \mathrm{C}$ & $1 \mathrm{~min}, 95^{\circ} \mathrm{C}$ \\
\hline Exposure Dose & $\begin{array}{l}90 \mathrm{~mJ} / \mathrm{cm}^{2}, \text { soft con- } \\
\text { tact }\end{array}$ & $\begin{array}{l}90 \mathrm{~mJ} / \mathrm{cm}^{2}, \text { vacuum con- } \\
\text { tact }\end{array}$ \\
\hline Post Exposure Bake & $1 \mathrm{~min}, 95^{\circ} \mathrm{C}$ & $1 \mathrm{~min}, 95^{\circ} \mathrm{C}$ \\
\hline Developement & $20 \mathrm{~s}$ & $1 \mathrm{~min}$ \\
\hline Hard Bake & $15 \mathrm{~min}, 180^{\circ} \mathrm{C}$ & $15 \mathrm{~min}, 180^{\circ} \mathrm{C}$ \\
\hline
\end{tabular}

\subsection{Dry etch and deposition processes}

\subsubsection{Reactive Ion Etch (RIE)}

Almost all dry etch processes, whether chemical or physical, are plasma based (an exception is for example gas phase etching with $\mathrm{XeF}_{2}$ [90]). The advantage of using plasmas in thin film deposition and etching is that the highly energetic ions allow performing processes at much lower temperatures than without a plasma. This can be crucial, if materials with low melting temperatures (e.g. $660^{\circ} \mathrm{C}$ for aluminum) are involved in a fabrication process.

The plasmas used in dry etch processes are mostly radio frequency (13.56 MHz) or DC generated, with argon or oxygen as inert gases, in a parallel plate reactor (figure 3.5). 

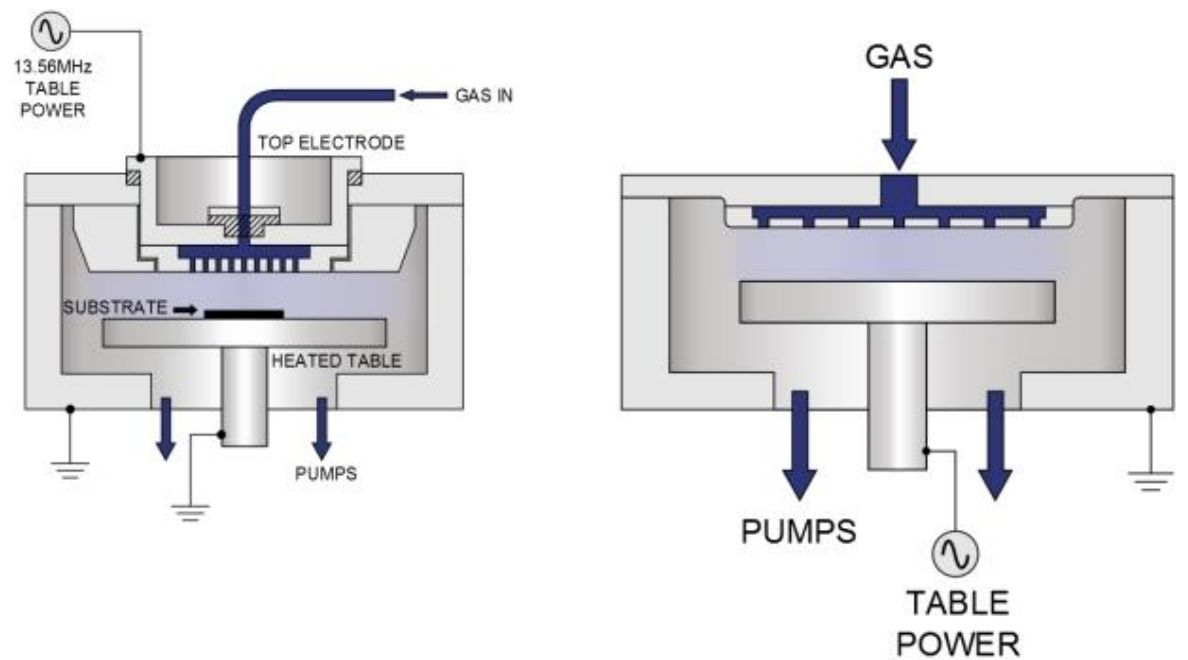

Figure 3.5: Reaction chambers for Plasma Enhanced Chemical Vapour Deposition (left) and Reactive Ion Etching (right) [91, 92].

Purly chemical dry etch processes have the advantage of great selectivity, because the gases react specifically with the selected material. In chemical dry etch processes, ions or reactive compounds that are generated by the plasma are brought to the surface of the sample where they adhere and react with the surface molecules. The gaseous reaction products diffuse back into the bulk gas [90]. Whenever good anisotropy is required, a pure chemical etching process is not the method of choice, because the reaction occurs isotropically at all exposed parts of the surface and the undercut (the amount of material that is etched under a mask) is therefore quite large.

Pure physical etching processes, such as sputter etching or ion beam milling, on the other hand, have a very good anisotropy, but a low selectivity and therefore an increased possibility of damaging the substrate. The high anisotropy in physical plasma etch techniques is the result of the bombardment of the surface with inert ions, e.g. argon ions, which cause the chemical bonds to break, leave the sample surface and redeposit on another surface, given that the mean free path is long enough to overcome a sufficiently large distance [90]. These ions are accelerated from the anode to the cathode, where the samples are placed, so that the ions arrive almost vertically at the surface. Physical plasma etch processes are very unselective, because the energy of the ions bombarding the surface is large compared 
to the chemical bond energies of the different materials of the sample.

Reactive Ion Etching (RIE) is a combination of physical and chemical etching. The sample surface is bombarded with reactive ions (halocarbons $\left(\mathrm{CHF}_{3}, \mathrm{CF}_{4}\right)$ or other halogen containing molecules $\left.\left(\mathrm{BCl}_{3}\right), \mathrm{SF}_{6}\right)$. This leads to sputter etching like in purely physical dry etch processes, but the reactive ions can also react with the surface material and, similar to the chemical dry etch processes, can be chosen in a way that the reaction with a specific material is preferred. This way, the ratio of the etch rates between the materials becomes larger [93] while the process is still anisotropic.

\subsection{Deposition processes}

\subsubsection{Plasma Enhanced Chemical Vapor Deposition (PECVD)}

During the deposition of thin films by PECVD, a reaction of gases, diluted in an inert gas (e.g. nitrogen or Argon), on or near the surface of a heated sample [90] takes place, where the components react to a solid material. Often these materials are dielectrics such as silicon nitride or silicon oxide, but even polymers such as PTFE-like materials can be deposited [94]. The reaction chamber differs from the one used for RIE processes only by a heating element (figures 3.5) which is necessary, because the reaction temperatures for PECVD processes is higher (20 ${ }^{\circ} \mathrm{C}$ for polymer deposition and $300^{\circ} \mathrm{C}-400^{\circ} \mathrm{C}$ for silicon nitride and silicon dioxide deposition).

PECVD was developed from APCVD (atmospheric pressure chemical vapor deposition), because deposition of dielectrics are often among the last steps of processes that involve temperature-sensitive components like metal circuit elements, and a possibility to perform these deposition processes at lower temperatures was needed. Although the operating temperatures of PECVD seem high compared to those used in RIE processes, they are considerably lower than in APCVD $\left(600^{\circ} \mathrm{C}-800^{\circ} \mathrm{C}\right)[95]$. The low temperatures are, however, also the cause of impurities in the deposited thin films, because byproducts stick to the surface and are incorporated into the films. This is the reason for a small amount of hydrogen or oxygen in PECVD 
silicon nitride layers that can alter its physical and chemical properties [95].

Other important methods of chemical material deposition that are widely used in microfabrication are electroplating (the electrochemical deposition by reducing metal salts, best known for nickel deposition from $\mathrm{NiCl}_{2}$ and silver deposition from $\mathrm{AgNO}_{3}$ ) and thermal growth, especially of silicon dioxide, which can be obtained in a very good quality by thermal oxidation of silicon at high temperatures $\left(>900^{\circ} \mathrm{C}\right)$.

\subsubsection{Deposition of metals}

The thermal evaporation of metals is one of the oldest deposition methods and was used in this work for the deposition of silver and gold. The metals are heated up to their boiling or sublimation point by passing a high current (for the silver and gold deposition using the Leybold Univex 100 about $180 \mathrm{~A}$ ) through a highly conductive trugsten boat which contains the metal.

Electron beam evaporation was used for deposition of the titanium adhesion layers. An electron beam is magnetically steered on a crucible of the required metal which evaporates locally and redeposits on the sample surface. The electron beam is accelerated by a high voltage (here: $7 \mathrm{kV}$ ) [96].

\section{Process parameters for dry etch and deposition processes}

In this work, RIE was used in two steps: for removing silicon nitride before the $\mathrm{KOH}$ etch to generate the four pore areas (section 3.10), and for etching the pores (section 3.11). In both cases SU-8 2001 was used as an etch mask. The process parameters were the same for both processes, except for the etching time. As the pores are much smaller than the pore areas, the reason for the smaller etch rate could be that the reaction products cannot diffuse away from the surface as fast as they can if a larger area is available. The RIE and PECVD processes were carried out with a PlasmLab System 100 (Oxford Instruments, Bristol, UK). After patterning the respective structures with photolithography, the substrates were etched without further cleaning. The process parameters were are shown in table 3.2 (RIE) and 3.3 (PECVD). 
Table 3.2: Process parameters for RIE processes.

\begin{tabular}{|l|l|l|}
\hline Parameters & $\begin{array}{l}\text { pore area etching (section } \\
3.10)\end{array}$ & pore etching (section 3.11) \\
\hline \hline Gases & $\mathrm{CF}_{3}(50 \mathrm{sccm})$ & $\mathrm{CF}_{3}(50 \mathrm{sccm})$ \\
& $\mathrm{O}_{2}(5 \mathrm{sccm})$ & $\mathrm{O}_{2}(5 \mathrm{sccm})$ \\
\hline Temperature & $20^{\circ} \mathrm{C}$ & $20^{\circ} \mathrm{C}$ \\
\hline Pressure & $50 \mathrm{mTorr}$ & $50 \mathrm{mTorr}$ \\
\hline Time & $8 \mathrm{~min}$ & $25-30 \mathrm{~min}$ \\
\hline Fwd Pwr & $151 \mathrm{~W}$ & $151 \mathrm{~W}$ \\
\hline DC Bias & $530 \mathrm{~V}$ & $530 \mathrm{~V}$ \\
\hline
\end{tabular}

Table 3.3: Process parameters for PECVD silicon nitride deposition.

\begin{tabular}{|l|l|}
\hline Parameters & $\begin{array}{l}\text { pore area etching (section } \\
3.10)\end{array}$ \\
\hline \hline Gases & $5 \% \mathrm{SiH}_{4} / \mathrm{Ar}(400 \mathrm{sccm})$ \\
& $\mathrm{NH}_{3}(30 \mathrm{sccm})$ \\
& $\mathrm{N}_{2}(780 \mathrm{sccm})$ \\
\hline Temperature & $300^{\circ} \mathrm{C}$ \\
\hline Pressure & 1000 mTorr \\
\hline Time & 32 min per side \\
\hline Fwd Pwr & $19 \mathrm{~W}$ \\
\hline
\end{tabular}

For thermal and electron beam evaporation, a Univex 350 vacuum deposition machine (Oerlikon Leybold Vacuum, Köln, GE) was used. Titanium (10 nm in thickness) was deposited as an adhesion layer for silver and gold at a rate of $2 \AA / \mathrm{s}$ and a power of $45 \%$. Silver $(150 \mathrm{~nm})$ was deposited at a rate of $5 \AA / \mathrm{s}$ and gold $(100 \mathrm{~nm})$ at a rate of $1 \AA / \mathrm{s}$, both at a power of about $45 \%$, which results in a current of about $180 \mathrm{~A}$. 


\subsection{Silicon etching in alkaline solution}

Silicon etching with alkaline solutions was developed in the early 1960s and became one of the most widely used silicon wet etch techniques. The most prominent advantages of this technique are its anisotropic and well predictable etching behavior, the possibility of batch processing and the inexpensive reagents and equipment. In a single crystal silicon the etch rate for alkaline solutions is different for each crystallographic plane and as a result the etching behavior is anisotropic. Although the reason of this behavior is still under debate, the etching characteristics of differently oriented silicon crystals have been studied extensively [97, 98].

I. Zubel et al. found that for (100) oriented silicon wafers (i.e. the (100) plane is perpendicular to the wafer plane) the etch rate in pure $\mathrm{KOH}$ solution is faster for (110), (331) and (221) planes than for (100) and (111) planes [99, 100]. K. Sato et al. determined the concentration dependent etch rates for various crystallographic planes [101]. The characteristic pyramidal shape of holes etched in (100) silicon (figure 3.6) is the result of the slow etch rate of the (111) plane, which lies at an angle of $54.74^{\circ}$ to the silicon wafer surface (figure 3.6) and is about 100 times slower than for any other crystallographic plane in pure KOH solution [102, 101].
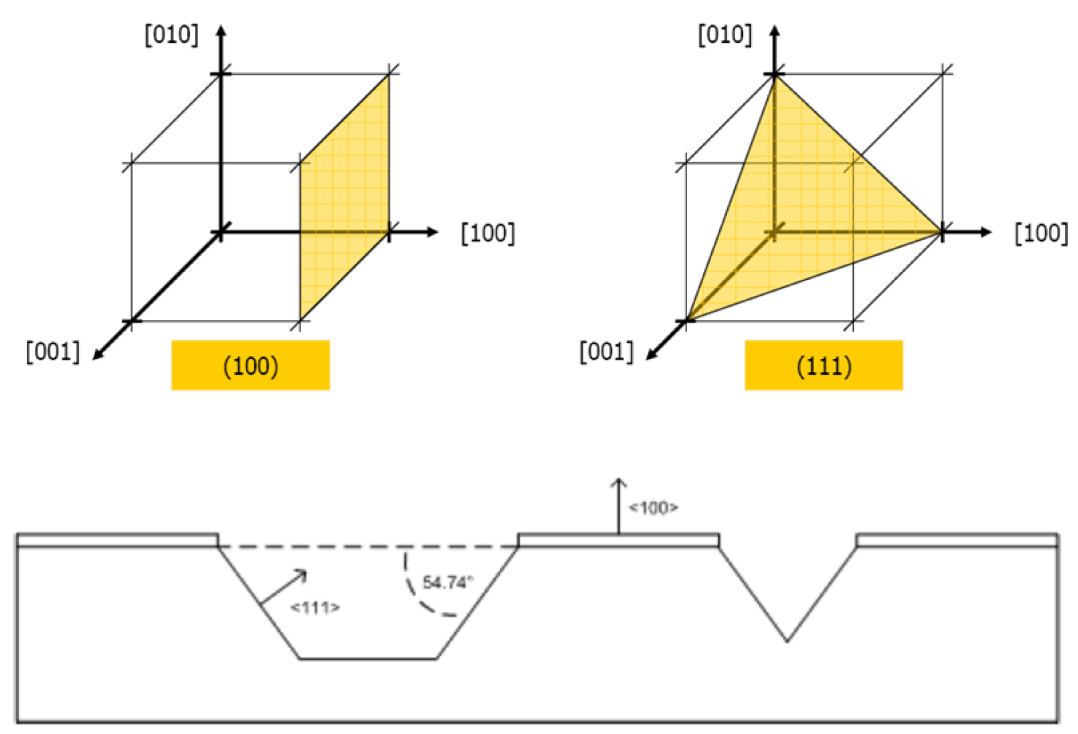

Figure 3.6: The etch rates in the $\mathrm{KOH}$ etch porcess of single crystal silicon depend on the crystal layer. The (111) layer is etched significantly slower than the (100) layer. 
The etch rate and the quality of the etched structures depend on the concentration of the etchant, the reaction temperature and on the presence and concentration of additives. All silicon wet etch solutions are alkaline solutions, either organic (e.g. hydrazine) or inorganic $(\mathrm{KOH}$ or $\mathrm{NaOH})$. The most commonly used etchants today are pure $\mathrm{KOH}$ solutions and $\mathrm{KOH}$ solutions with a small percentage of either IPA (isopropyl alcohol) or TMAH (tetramethylammonium hydroxide). The reaction equation for all etching solutions was found to be $\mathrm{Si}+2 \mathrm{OH}^{-}+2 \mathrm{H}_{2} \mathrm{O} \rightarrow$ $\mathrm{SiO}_{2}(\mathrm{OH})_{2}^{2-}+2 \mathrm{H}_{2}$ [97].

The etch rate for silicon in pure $\mathrm{KOH}$ increases at first with increasing concentration up to $4 \mathrm{M}$ and then decreases. H. Seidel et al. found in a study of the reaction kinetics the relation $R=k_{0}\left[\mathrm{H}_{2} \mathrm{O}\right]^{4}[\mathrm{KOH}]^{1 / 4} e^{-E_{\alpha} / k T}$ to be the best fit for this behavior for all concentrations [97]. The addition of IPA and TMAH decreases the etch rate in general, but the effect is different for each crystallographic plane. The addition of IPA, for example, leads to a $20 \%$ decrease for the etch rate of the (100) plane and of $90 \%$ for the (110) plane and therefore reverses the etch rate ratio for these two planes [97].

Both temperature and concentration of the etchant have an influence on the quality of the resulting structures. In a study by M. H. Yun et al. the quality of a sharp tip etched in a silicon-on-insulator wafer is shown for various temperatures and $\mathrm{KOH}$ concentrations [103]. The best result was achieved with $30 \% \mathrm{KOH}$ solution at $70^{\circ} \mathrm{C}$, which is similar to the temperature and concentration used in this work $\left(30 \%, 85^{\circ} \mathrm{C}\right)$.

\section{Experimental procedure of the $\mathrm{KOH}$ etch}

After patterning the four squares on the backside of the substrate (chapter 3.6) they were placed in a teflon holder. The holder was immersed in a glass beaker containing the $30 \% \mathrm{KOH}$ solution which was heated to $85^{\circ} \mathrm{C}$. To avoid a change in the concentration of the $\mathrm{KOH}$ solution due to the evaporation of water, the beaker was covered with two layers of Parafilm $(\mathrm{B} M$. According to H. Seidel el al. the etch rate of (100) silicon is about $1 \mu \mathrm{m} / \mathrm{min}$ [97] and the reaction time for the $275 \mu \mathrm{m}$ thickness of the substrate therefore about $4.6 \mathrm{~h}$. To ensure the complete etching of the silicon underneath the silicon nitride membrane, the reaction was stopped after $6 \mathrm{~h}$. No underetching of the silicon nitride on the backside was observed after 
the reaction. Figure 3.7 shows one pore area at various states of etching. At the end of the etching process the pure silicon nitride membrane (blue in figure 3.7) remains and can clearly be distinguished from the structured silicon.
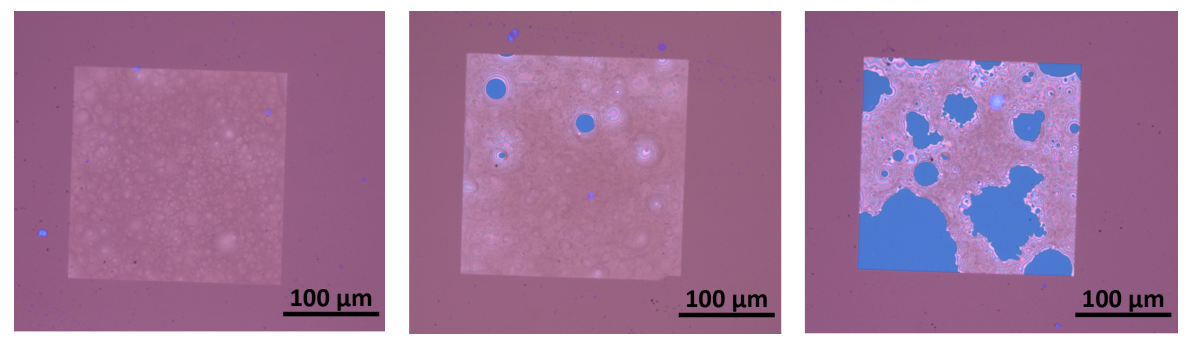

Figure 3.7: The $\mathrm{KOH}$ etch of the silicon nitride membrane at three different stages of the etch process. The pure silicon nitride membrane appears at the end of the etch porcess as a flat layer, whereas the silicon is structured due to the crystal layer dependent etch process.

\subsection{Design considerations}

The microporous substrate and the sample chamber had to meet several demands:

1. The possibility of performing multiple experiments on one substrate

2. Easy handling

3. Suitability for a wide variety of experimental techniques

4. Exchange of solutions during the experiment

The possibility of performing multiple experiments on one substrate has several advantages. The experiment can be continued even if one lipid bilayer ruptures so that the overall measurement time can be increased and the data output is higher than in experiments which rely on the success of only one lipid bilayer formation, especially as the substrates have to be cleaned very thoroughly before another lipid bilayer can be prepared on an already used pore-array. For this reason the microporous substrate has four porous arrays, which are individually addressable and have separate electrolyte compartments and electronic connections.

By having separated electrolyte compartments, which are connected to tubing, the 
exchange of solutions or introduction of chemicals throughout the whole experiment is possible. The substrate offers the possibility to use integrated electrodes on the front side, which are deposited directly onto the substrate, to be able to measure the current in close proximity to the porous arrays and at the same time provide access to the array for a microscope objective with a low working distance. Integrated electrodes make it easy to switch the electrical connections, e.g. between the impedance spectrometer and the amplifier, that is used for single ion channel recording, without disturbing the experiment.

Different experimental techniques require different substrate properties. For optical microscopy, large porous arrays with large pore-sizes are used because often a large area of free-standing lipid bilayer is neccessary, and the refraction limit makes imaging of nanometer-sized pores impossible.

For electrical experiments, especially ion-channel recordings, an electrically insulating lipid bilayer is required which is mainly generated by using a small number of pores with very small diameters.

To have the possibility of using the same substrate for both optical microscopy and electrical measurement techniques, the pore diameter was chosen to be $1 \mu \mathrm{m}$ and the number of pores was limited to 9 pores per array for the electrical experiments.
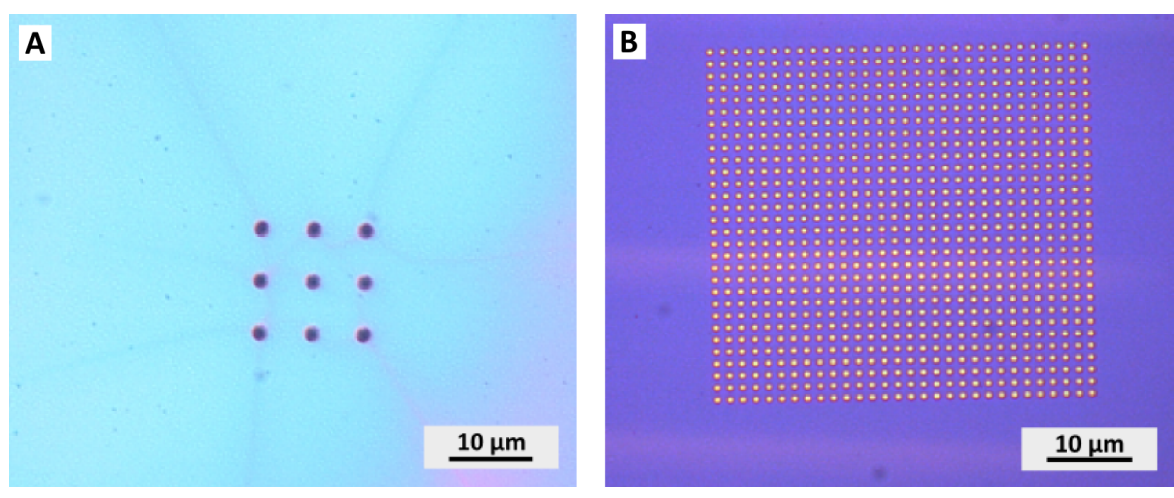

Figure 3.8: Porous arrays with $\mathrm{N}=9(\mathbf{A})$ and $\mathrm{N}=900$ pores $(\mathbf{B})$

In a first approach, each pore-array contained 900 pores $(30 \times 30$ pores with a total pore area of $88 \mu \mathrm{m}$ x $88 \mu \mathrm{m}$, figure $3.8, \mathrm{~B})$, but it turned out to be impossible to cover the whole array with a lipid bilayer generated by spreading of Giant Unilamellar Vesicles (GUVs), because on vesicle cannot cover the whole array and 
they do not fuse neatly so that gaps between the individual vesicles remain (section 5.3). Therefore, the total pore-area had to be significantly reduced. As the average diameter of the GUVs is about $10 \mu \mathrm{m}$ and therefore the area of the flat GUVs about $315 \mu \mathrm{m}^{2}$, a pore array of only $10 \mu \mathrm{x} 10 \mu \mathrm{m}$ containing 9 pores was chosen (figure 3.8, A). The substrates containing 900 pores, however, are very useful whenever experiments without the need for an electrically insulating lipid bilayer are performed, for example fluorescence microscopy experiments, where the only requirement is a large area of mostly membrane-covered pores. The number of pores is easily adjustable by changing the photomask in step 3 of the fabrication process (section 3.6) so that the substrate can be prepared according to the requirements of the experiment.

Apart from the difficulties concerning the preparation of electrically insulating lipid bilayers, several fabrication strategies had to be tried before substrates with a satisfyingly low surface roughness and pore shape were found. Figure 3.9 shows three different approaches. In the first approach (figure 3.9, A) the SU-8 photoresist layer remained on the substrate to act as a passivation layer (chapter 4.4.1). In the second approach (figure 3.9, B) a silicon dioxide layer was deposited after the poreetching step (section 3.6) which resulted in a smooth surface, but also in conically shaped pores, which were considered not suitable for lipid bilayer preparation. The third approach (figure 3.9, C) which was then used for the experiments, resulted in a smooth surface and cylindrically shaped pores.

The detailed fabrication scheme of the approach shown in figure 3.9, $\mathbf{C}$ that was used for the experiments, is discussed in this chapter. A detailed characterization of all approaches will be given in chapter 4 . 
(A)

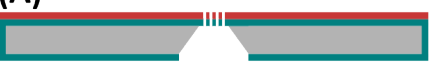

(B)

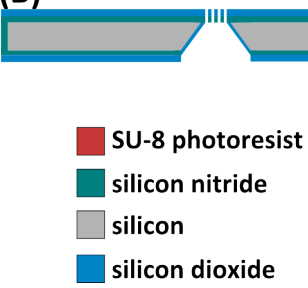

(C)

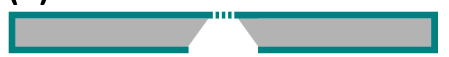

Figure 3.9: Cross sections of the three different approaches for the substrate fabrication. In $\mathbf{A}$ the $\mathrm{SU}-8$ photoresist remains on the substrate, in $\mathbf{B}$ a silicon dioxide layer for passivation was deposited, resulting in conically shaped pores. $\mathbf{C}$ shows the final approach, resulting in a low surface roughness and cylindrically shaped pores (chapter 4).

\subsection{Fabrication scheme}

\section{Substrate preparation and dicing}

The porous substrates were prepared from double side polished, (100) oriented, n-type silicon wafers, $4 "$ in diameter and $275 \mu \mathrm{m}$ in thickness (Si-Mat Silicon Materials, Kaufering, GE). In the first step the wafers are diced into $22 \mathrm{~mm}$ x $26 \mathrm{~mm}$ substrates (figure 3.10, (1)), which are cleaned with a solution of $\mathrm{NH}_{3} / \mathrm{H}_{2} \mathrm{O}_{2} / \mathrm{H}_{2} \mathrm{O}$ $(1 / 1 / 5,30 \mathrm{~min})$ in an ultrasonic bath. Additionally, the substrates are rinsed with $\mathrm{ddH}_{2} \mathrm{O}$, acetone and isopropanol and cleaned in $\mathrm{O}_{2}$-plasma for several minutes before silicon nitride deposition.

\section{Structuring of the pore areas}

A $300 \mathrm{~nm}$ silicon nitride layer was deposited on both sides of the substrate in a PECVD process (figure 3.10, (2)) using 5\% $\mathrm{SiH}_{4} / \mathrm{Ar}$ (400 sccm) as silicon source and $\mathrm{NH}_{3}(30 \mathrm{sccm})$ as the nitrogen source.

The silicon nitride layer was patterned with SU-8 2001 photoresist (MicroChem, Newton, US, figure 3.10, (3)) and removed in the four square areas $(640 \mu \mathrm{m} x$ $640 \mu \mathrm{m})$ in a fluorine based RIE process $\left(\mathrm{CH}_{3}(50 \mathrm{sccm}), \mathrm{O}_{2}(5 \mathrm{sccm})\right)$ on the back side of the substrate (figure 3.10, (4)).

In the next step, a KOH wet etch process was used to etch the silicon in the four pore areas (chapter 3.4). The substrates were immersed in a $30 \% \mathrm{KOH}$ solution 
and the silicon in the four areas was etched at $85{ }^{\circ} \mathrm{C}$ leaving four silicon nitride layers (squares of $250 \mu \mathrm{m} \times 250 \mu \mathrm{m}$ ) on the front side (figure 3.10, (5)).

Silicon nitride has a negligibly small etching rate in $\mathrm{KOH}$ [97] which makes it an excellent etch mask in alkaline wet etch processes. For this reason, the silicon nitride layer on the back side of the substrate was used as a protection mask for the silicon underneath. It was necessary to use silicon nitride as a protection layer, because several other materials that were tested for this purpose failed. Using SU8 directly as a protection layer, which would be convenient because it is used for patterning the four squares anyway, does not work, because the photoresist delaminates after 30-60 min in the KOH solution. Apiezon Wax W ("Black Wax", M\&I Materials Limited, Manchester, UK), which is often used in MEMs applications to protect already existing structures during $\mathrm{KOH}$ etch, melts at temperatures higher than $70^{\circ} \mathrm{C}$, and performing the process at such low temperatures increases the etching time considerably. Commercially available protective coatings such as ProTeK@B3 (Brewer Sciences, Rolla, MO, USA) are very expensive, require additional patterning steps and are not easily removable. Mechanical protections like clamps or chambers, that expose only the part of the substrate that is supposed to be etched, do not allow processing many substrates at a time or complicated etch patterns.

When the silicon in the four pore areas is etched completely, only the silicon nitride layer on the front side remains. As it is not etched at all [99], its thickness is considered to be $300 \mathrm{~nm}$, also after the $\mathrm{KOH}$ etching step. 

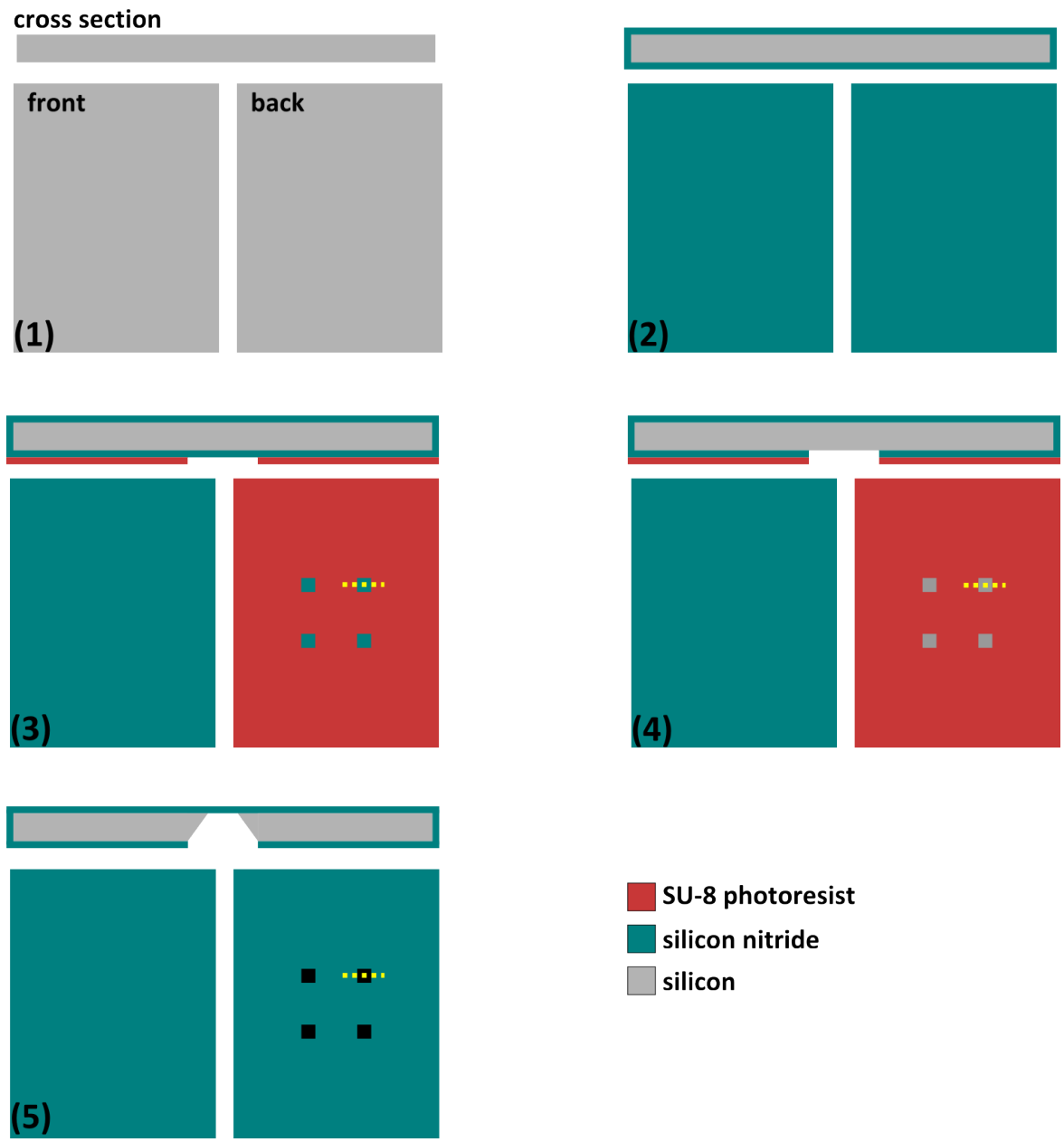

Figure 3.10: In step 1 of the fabrication process the silicon wafer is diced (1) and a PECVD silicon nitride layer is deposited on both sides (2). In step 2 the pore areas are patterned $(\mathbf{3})$ and the silicon nitride is removed from four squares on the back side (4). The bare silicon is etched with $\mathrm{KOH} \mathrm{(30 \% )} \mathrm{so} \mathrm{that} \mathrm{four} \mathrm{silicon}$ nitride membranes remain on the front side $(\mathbf{5})$.

\section{Structuring of the pores}

In step 3, the pores are patterned and etched into the silicon nitride membrane on the front side (figure 3.11). Pore-arrays of different sizes and pore numbers can be fabricated: In a first approach, an $88 \mu \mathrm{m}$ x $88 \mu \mathrm{m}$ array with 900 pores was patterned, but later a design with a porous region of $10 \mu \mathrm{m} \times 10 \mu \mathrm{m}$ containing 9 pores was used in the experiments. In both types of arrays the pores have a 
nominal diameter of $1 \mu \mathrm{m}$. This diameter was chosen because of the resolution limit of the mask aligner (Karl SUSS MJB4, SÜSS MicroTec AG, Garching, GE) of $0.8 \mu \mathrm{m}$. Larger diameters would have decreased the stability of the lipid bilayers. The pores were again patterned using SU-8 2001 photoresist (figure 3.11, (1)) and the silicon nitride in the pores was removed in the same RIE process used in step 2 so that holes in the silicon nitride layer on the front side were formed (figure 3.11, (2)). The SU-8 photoresist was removed from the front side by immersing the substrates again in 30\% $\mathrm{KOH}$ for $30-60 \mathrm{~min}$ at $65^{\circ} \mathrm{C}$ (figure 3.11, (3)), cleaning them in $\mathrm{H}_{2} \mathrm{O} / \mathrm{H}_{2} \mathrm{O}_{2} / \mathrm{NH}_{3}(5 / 1 / 1)$ solution and treated 10 min in oxygen plasma.
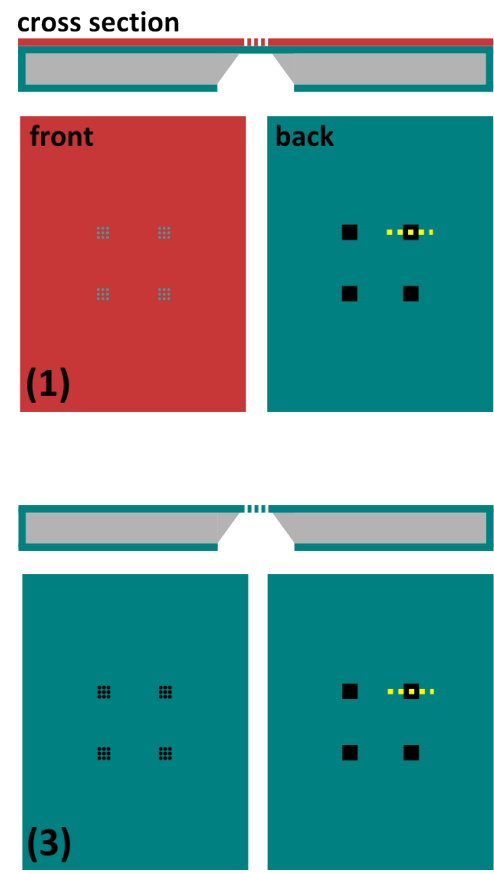

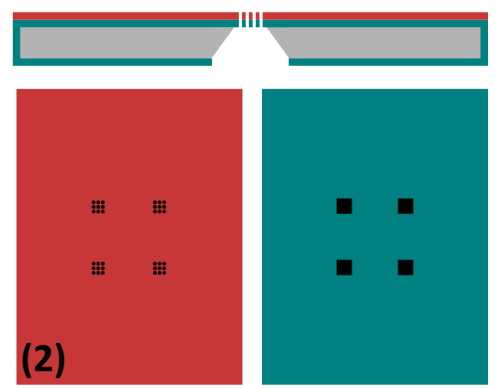

SU-8 photoresist

silicon nitride

silicon

Figure 3.11: In step 3 the pores $(1 \mu \mathrm{m}$ in diameter $)$ are patterned using SU-8 photoresist (1) and etched in RIE process (2). The photoresist is delaminated from the substrate with KOH solution (3).

\section{Silver electrode deposition}

For the electrodes a silver layer of $150 \mathrm{~nm}$ thickness with a titanium adhesion layer of $10 \mathrm{~nm}$ was deposited on the front side of the substrate with thermal and electron beam evaporation respectively. Custom-fabricated aluminum masks were used to 
pattern the electrodes so that the electrode on the front side was formed. The electrodes were chlorinated before the experiment by incubating the substrate in $10 \% \mathrm{NaOCl}$ for $3 \mathrm{~min}$.
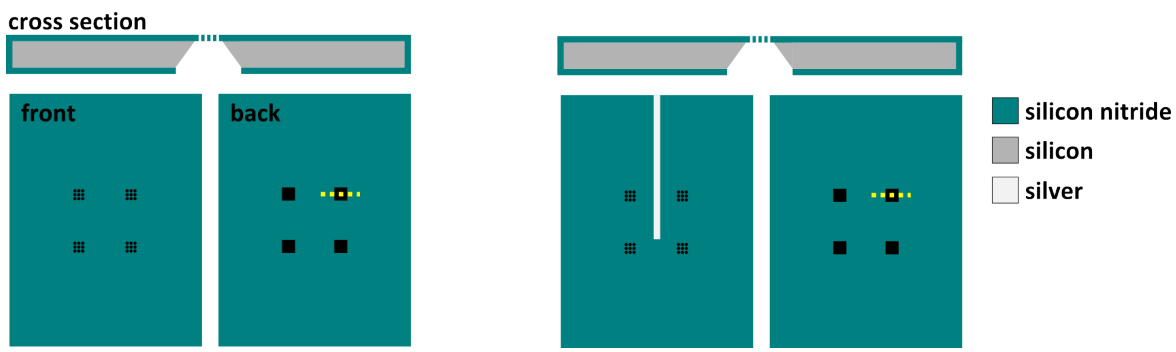

Figure 3.12: In step 3 the integrated electrode $(1 \mathrm{~mm}$ in width and $150 \mathrm{~nm}$ in height) is deposited on the substrate by thermal evaporation of silver. For better adhesion a layer of titanium ( $10 \mathrm{~nm}$ in height) is deposited before by electron beam evaporation.

Alternative methods for the deposition and patterning of the integrated silver electrode are lift-off processes, where a photoresist layer is patterned first, leaving only the shape of the electrode exposed which is then removed together with the remaining metal (figure 3.13). It is also possible to deposit the silver on the whole substrate first and then pattern a photoresist protection layer for the electrode structure. The remaining silver is then etched in a $\mathrm{H}_{2} \mathrm{O} / \mathrm{H}_{2} \mathrm{O}_{2}$ solution and the photoresist protection layer is removed at the end (figure 3.13).

lift-off
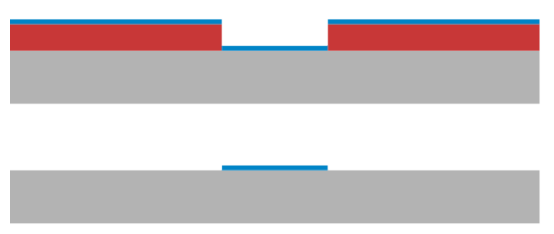

protection layer

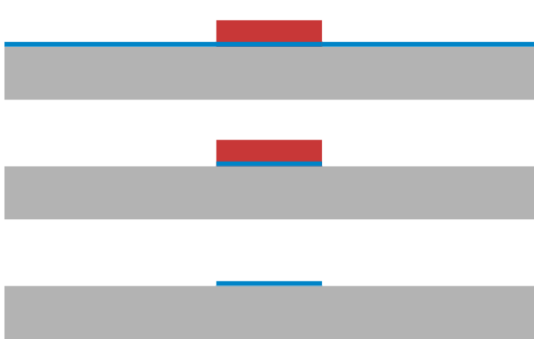

Figure 3.13: Alternative methods for silver electrode generation are lift-off processes (left) or the use of photoresist protection layers (right).

Both methods were tried, but although the resulting structures are slightly less rough than those obtained with the mechanical mask, they both require the depo- 
sition of either silver or photoresist into the previously etched pores which leads to impurities that require another extensive cleaning step, whereas the mechanical mask protects the arrays during the whole evaporation process. Also, both processes contain two additional steps. In many cases the silicon nitride layers were damaged during the processing or the cleaning step.
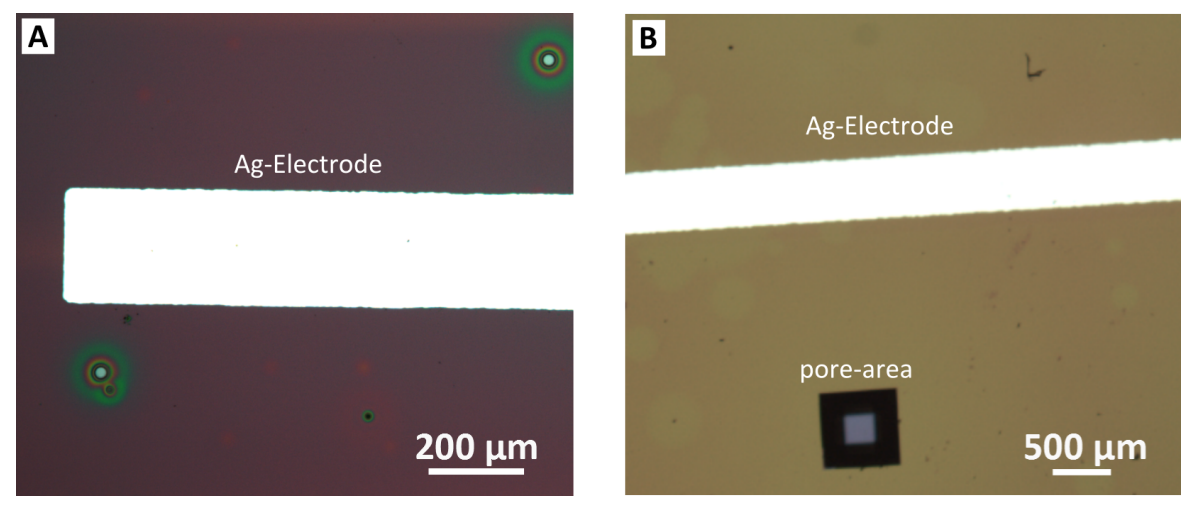

Figure 3.14: Silver electrodes obtained by using a photoresist protection layer $(\mathbf{A})$ and a mechanical aluminum mask $(\mathbf{B})$. 


\section{CHAPTER 4}

\section{Characterization of the substrate}

In order to determine if the microporous substrate is suitable for membrane formation, it was characterized in terms of surface roughness and the properties of the etched pores.

Atomic force microscopy (AFM) was used to estimate the surface roughness of the silicon nitride membrane. A low surface roughness is crucial for the lipid bilayer formation and it is of essential importance if the lipid bilayer has to be electrically insulating because any roughness can lead to defects in the lipid bilayer and therefore to leak-currents that disturb the electrical measurements.
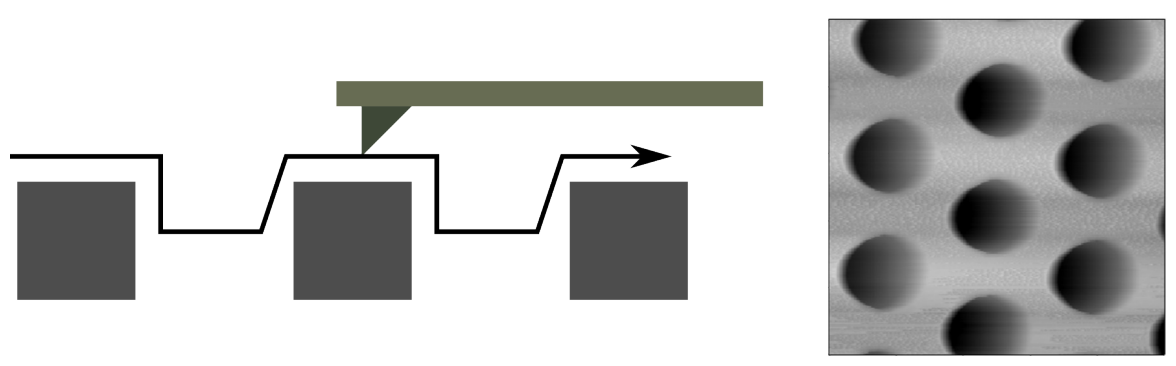

Figure 4.1: Atomic force microscopy provides detailed information on the surface roughness, but when imaging holes with a large diameter, artifacts can occur.

Although AFM is a very good instrument to examine the surface roughness of the substrate, for the pores themselves this technique is not so well suited, because imaging the relatively large and deep holes leads to artifacts when the cantilever tip scans across a pore (figure 4.1). However, not only a low surface roughness, but 
also smooth pore-rims are necessary to avoid defects and uncovered pores during membrane preparation. In order to obtain this information, the pores were imaged with Scanning Electron Microscopy (SEM). That way, the shape of the pores can be examined more accurately.

Additionally, the radius and the roundness of the pores were determined using brightfield microscopy images.

\subsection{Determination of pore-radius and roundness}

For the determination of the mean pore radius, brightfield microscopy images of the 900 and 9 pore arrays like the one shown in figure $4.2 \mathrm{~A}$ were used. With the software ImageJ, a pixel analysis of the images was performed. A threshold was automatically adjusted to the area of the pores (figure $4.2 \mathrm{~B}$ ). For these pixels, the feret-diameter (i.e. the distance between the two points farthest apart from each other) and the roundness were determined.
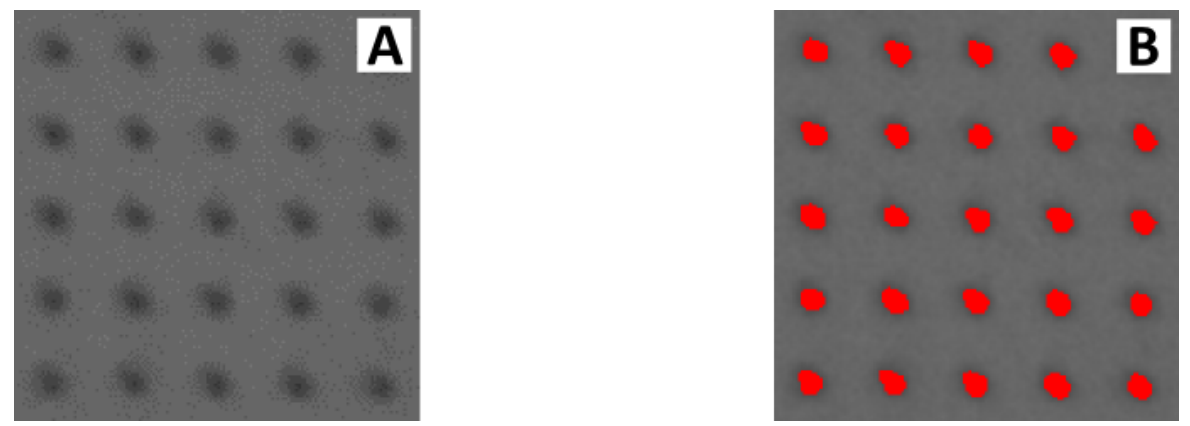

Figure 4.2: The feret-diameter and the roundness of the pores was estimated by a pixel analysis of brightfield images $\mathbf{A}$. A threshold was adjusted to the pores and the area was calculated $\mathbf{B}$.

By this process, the roundness of the pores was estimated to $(89 \pm 4) \%$ and the diameter to $(1.18 \pm 0.14) \mu \mathrm{m}$. Brightfield microscopy images were used instead of SEM images, because here more data were available since not so many SEM images of the substrates were taken. For comparison, the same process was used for the SEM images, which gave diameters between $0.9 \mu \mathrm{m}$ and $1.4 \mu \mathrm{m}$, showing good agreement with the values obtained for the brightfield images. 


\subsection{Atomic Force Microscopy}

Atomic force microscopy (AFM) is a scanning microscopy technique which evolved from Scanning Tunneling Microscopy in the 1980s and was first described by G. Binnig, C.F. Quate and C. Gerber [104]. It became one of the most important high-resolution imaging techniques for biological samples, because it allows imaging non-conducting samples in physiological environments (i.e. in aqueous solution at ambient temperature)[105].

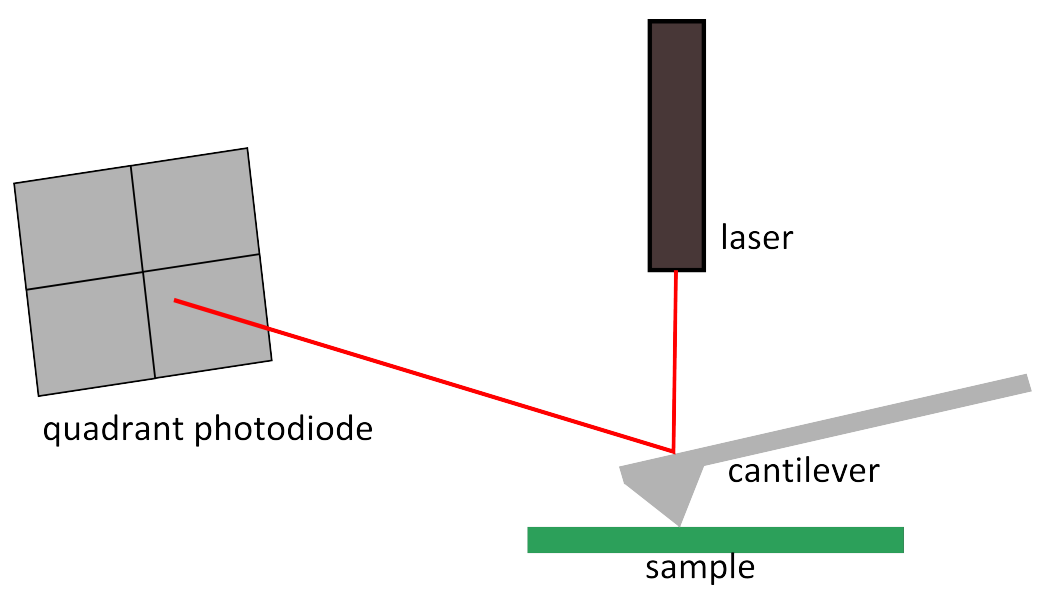

Figure 4.3: Working principle of the AFM. A sharp tip scans across the surface of a specimen. The changes in topography are detected by a laser beam that is reflected onto a quadrant photo diode.

Figure 4.3 shows the working principle of the AFM. A sharp tip (average diameters are in the order of $10 \mathrm{~nm} \mathrm{[106]),} \mathrm{attached} \mathrm{to} \mathrm{a} \mathrm{cantilever,} \mathrm{acts} \mathrm{as} \mathrm{a} \mathrm{probe} \mathrm{scanning}$ across the surface in xy-direction driven by piezo actuators. The degree of the cantilever bending changes whenever the height of the surface changes so that the interaction between the cantilever tip and the surface provides information on its topography.

For detection, a laser beam is focused on the cantilever, where it is reflected to a quadrant photo diode. A change in the cantilever bending also causes a change in the signal on the photo diode. Because biological samples are prone to damage, the cantilever is usually driven at a low constant force, although, of course, also constant height measurements are possible. To move the cantilever at a constant 
force, the signal from the photo diode is used as a feedback and the height is adjusted by the z-piezo element.

In the widely used contact mode, the cantilever moves across the surface with a given force (set point) that can be adjusted manually between surface and tip. When this force changes due to alterations in the height of the sample, the difference is compensated so that the original force is restored.

In tapping mode, or intermittent contact mode, the cantilever is oscillated by an additional piezo element near its resonant frequency so that the change of the signal is due to a damping of the amplitude of this oscillation when cantilever and tip come into contact. In contact mode, loosely attached or very soft samples may be dragged across the surface by the cantilever leading to damage of the sample or image artifacts, which is prevented in tapping mode. Therefore, the advantage of tapping mode is an improvement in lateral resolution and less damage of the samples [105].

\section{Experimental Procedure}

The experiments were carried out with a Cypher AFM (Asylum Research, Santa Barbara, CA). The estimation of the surface roughness was performed in tapping mode in water using a cantilever (DNP-S, Bruker AFM Probes, Camarillo, CA) with a nominal spring constant of $0.35 \mathrm{~N} / \mathrm{m}$ and $0.24 \mathrm{~N} / \mathrm{m}$, respectively, and a nominal resonance frequency of $65 \mathrm{kHz}$ or $56 \mathrm{kHz}(33 \mathrm{kHz}$ and $18 \mathrm{kHz}$ in water). All samples were cleaned with $\mathrm{H}_{2} \mathrm{O}_{2} / \mathrm{NH}_{3} / \mathrm{H}_{2} \mathrm{O}\left(1: 1: 5,10\right.$ min $\left.75^{\circ} \mathrm{C}\right)$ prior to the experiments.

\subsection{Electron microscopy}

Electron microscopy uses the interaction of electrons with the material of a sample for image generation. Two classes of interactions can occur when an electron hits the surface of a specimen: elastic and inelastic interactions (figure 4.4). When an electron is scattered elastically, no energy, or only a negligibly small amount of energy, is transferred from the primary electrons of the beam to the atoms of the sample. No energy is transferred when the electrons pass through the sample without any interaction with the material at all. Those electrons contribute to 
the direct beam which contributes most to the background noise. Electrons can also be deflected by Coulomb interaction without a significant energy loss, which is the case, when the electrons are attracted by the positive potential of the nucleus of an atom. The smaller the distance between the electron and the nucleus, the larger the deflection. Also complete backscattering of the electron can occur. This type of interaction is mainly used for Transmission electron microscopy (TEM). Backscattered electrons provide information on the chemical composition of the sample, because with an increasing atomic number more electrons are backscattered and thus regions with a high density of backscattered electrons appear lighter on the image. Diffraction imaging uses backscattered electrons to determine the crystal structure near the surface [107].

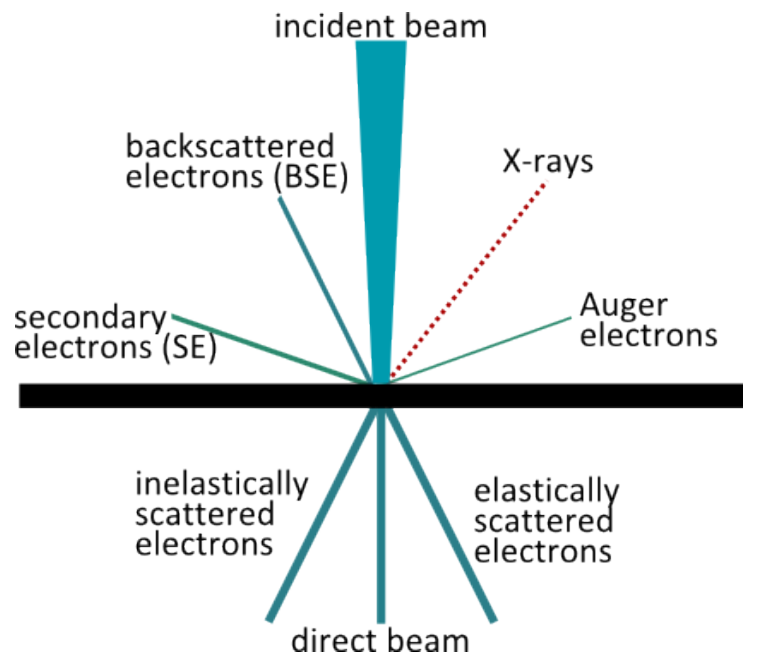

Figure 4.4: Electrons can interact elastically and inelastically with matter. Elastic interaction leads to elastic scattering, the direct beam and backscattering. Inelastic interactions include the generation of secondary electron, Auger electrons and X-rays.

Inelastic scattering occurs when energy is transferred from the primary electrons to the atoms of the sample material. When an incident electron transfers a part of its energy to an electron in an atom of the specimen, this electron can be promoted to a higher shell or a higher energy level. To reach the more favorable ground state again and fill the vacancy in the inner shell, an electron has to lose some of its energy again, which can occur by emitting characteristic X-ray radiation. This type of interaction is used in SEM and it provides information on the chemical 
consistency of the sample surface, because the wavelength of the emitted X-rays is characteristic for each element.

Another possible process is the transfer of the excess energy of the excited electron to another electron, which can gain sufficient energy to be ejected from the atom. These ejected electrons are designated Auger electrons. Secondary electrons are low-energy electrons (less than $50 \mathrm{keV}$ ) ejected, for example, from the valence band of metal samples. SEM uses secondary electrons for generating images of the surface topography, because their generation is highly dependent on the properties of the surface. Edges appear lighter in SEM images, since less electrons are absorbed by the surrounding material $[107,108,109]$.

\section{Experimental Procedure}

The experiments were carried out with a Philips SEM 515 Scanning Electron Microscope (resolution: $50 \mathrm{~nm}$ ). All samples were cleaned with $\mathrm{H}_{2} \mathrm{O}_{2} / \mathrm{NH}_{3} \mathrm{H}_{2} \mathrm{O}$ (1:1:5, $10 \mathrm{~min} 75^{\circ} \mathrm{C}$ ) and a $10 \mathrm{~nm}$ gold layer was sputtered on the surface to avoid charge effects of the non-conducting silicon nitride surface prior to the experiment. The samples were mounted on a holder using adhesive carbon tape and the experiments were performed at voltages between 20 and $30 \mathrm{kV}$.

\subsection{Results and discussion of AFM and SEM experiments}

\subsubsection{SU-8 coated substrates}

In a first approach of the fabrication of the microporous substrates, the SU-8 photoresist that was used as a photomask for the pore-etching step (chapter 3.6) remained on the substrate so that lipid bilayer formation occurred on the SU8 surface. The $1 \mu \mathrm{m}$ thick SU-8 layer was supposed to act as a passivation layer, because its dielectric constant $\left(\epsilon_{S U-8}=3,[110]\right)$ is smaller than that of silicon nitride $\left(\epsilon_{S i N}=7.5,[111]\right)$.

Because SU-8 is a hydrophobic material and for lipid bilayer formation by direct spreading of GUVs without a functionalization a hydrophilic surface is required, the substrates were treated with conc. $\mathrm{H}_{2} \mathrm{SO}_{4}$ for 10 min prior to the experiment. 
According to S. Tao et al. [112] the acid leads to a ring-openig reaction of the epoxide rings of the SU-8 which renders the material hydrophilic (figure $4.5(\mathbf{A})$ ). The difference in hydrophilicity between the $\mathrm{H}_{2} \mathrm{SO}_{4}$-treated parts of a substrate and the non-treated can be observed for several hours when the substrates are stored in water.

To validate the possibility of lipid bilayer formation on SU-8 coated silicon nitride substrates, vesicles, doped with 2 mol\% BODIPY-C12-HPE, were spread on SU8 coated solid PECVD silicon nitride substrates and observed with fluorescence microscopy $(4.5(\mathbf{B}))$. The images show that GUVs adhere and rupture on the surface.
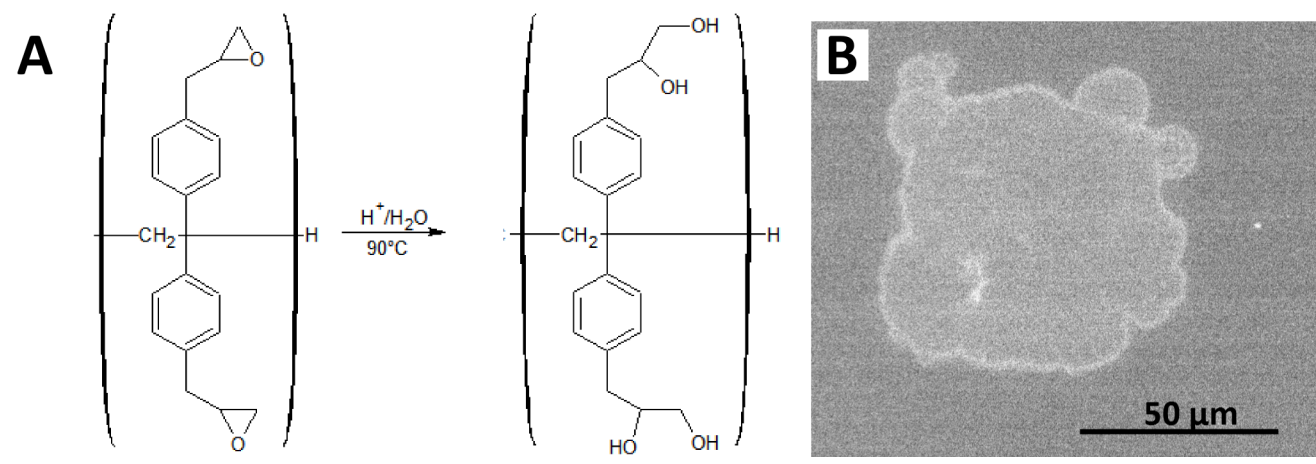

Figure 4.5: SU-8 photoresist can be rendered hydrophilic by an acid-catalyzed ring-opening reaction of the epoxide ring $(\mathbf{A})$ which makes lipid bilayer formation possible on solid supports (B).

The AFM images reveal round, bubble-like structures (figure $4.6(\mathbf{C})$ ) which are the reason for the high surface roughness. They are presumable caused by the exposure to oxygen plasma during the pore-etching step. Walther et al. examined SU-8 after exposure to oxygen plasma and found similar structures [113]. Also E. Bonaccurso and K. Graf showed the structuring effect of plasma on polymer materials [114]. As pointed out before, rough surfaces can lead to rupture of the lipid bilayers spanning the pores. For solid supported substrates, the surface roughness seems to have less influence on the integrity of the lipid bilayer, since none of the GUVpatches showed any damage in fluorescence microscopy images. The pore-spanning lipid bilayers, however, are much more sensitive to irregularities in the surface, especially at the border between supported and free-standing regions. Lipid bilayer formation was not possible on SU-8 covered porous substrates. Because in this 
approach only large pore-arrays with 900 pores were used, the main reason why no lipid bilayers can be formed is probably the incomplete coverage of the substrate as it has been explained in section 5.3.2. Nevertheless, also an extremely uneven surface can lead to rupture of the lipid bilayers.
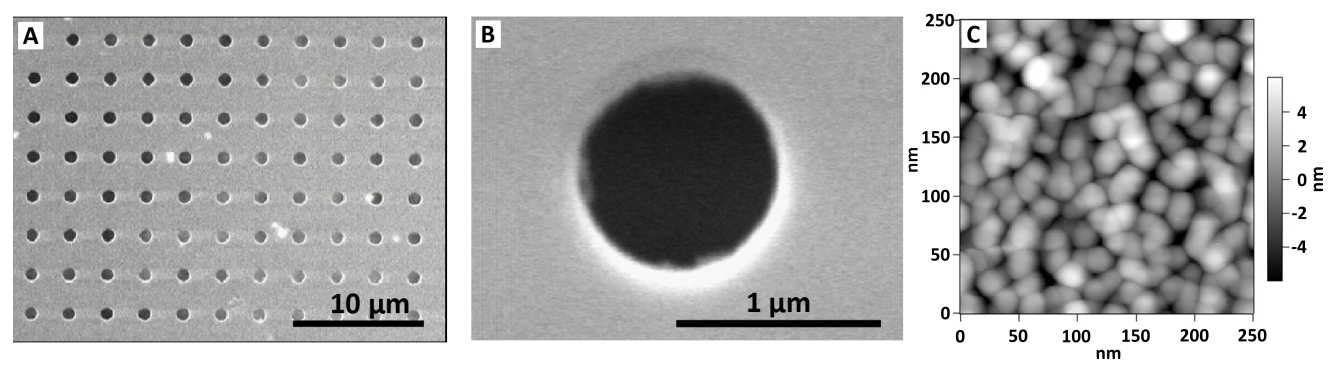

Figure 4.6: AFM and SEM images SU-8-based approach, which have properly etched pores $(\mathbf{A}, \mathbf{B})$, but a high surface roughness $(\mathbf{C})$.

Although the surface roughness was considered too high for lipid bilayer formation on the porous substrates, the SEM images show that the etching process in general results in well-defined pores with a diameter that is close to the nominal diameter of $1 \mu \mathrm{m}$ (section 4.1).

Because the oxygen plasma treatment cannot be avoided and removing as well as redeposition of the SU-8 leads to clogging of the pores, this appoach has been discarded, but the fabrication process in general was taken as a basis for the following approaches.

\subsubsection{Silicon dioxide coated substrates}

In the second approach PECVD-silicon dioxide was used as a passivation layer instead of SU-8. For this reason the SU-8 first had to be removed. SU-8 does not etch in $\mathrm{KOH}$ or any organic solvent, but only in $\mathrm{CF}_{4}$ containing oxygen plasma [110]. Although it does not etch, it delaminates in $30 \% \mathrm{KOH}$ solution, so the substrates were immersed in this solution and heated to $65^{\circ} \mathrm{C}$ to avoid further etching of the silicon and enhance the delamination process. The SU-8 delaminated completely within $60 \mathrm{~min}$.

After the SU-8 removal the substrates were cleaned in $\mathrm{H}_{2} \mathrm{O} / \mathrm{H}_{2} \mathrm{O}_{2} / \mathrm{NH}_{3}$ (5:1:1) solution and treated 10 min with oxygen plasma (section 3.6) before silicon dioxide 
deposition.
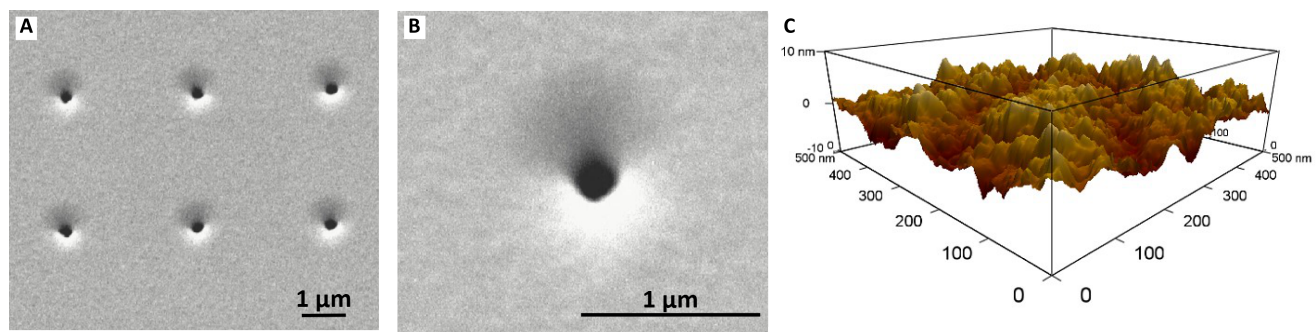

Figure 4.7: AFM and SEM images of the the silicon dioxide coated substrates that have a low surface roughness $(\mathbf{C})$, but cone-shaped pores $(\mathbf{A}, \mathbf{B})$.

The AFM images show that the surface roughness decreased significantly (figure $4.7, \mathrm{C})$ which shows that the previously observed rough surface is due to the SU-8. The SEM images, however, show that the pores now have a cone-shaped structure instead of the cylindrical one they had before. To rule out the possibility that the cone shape is not already a result of the pore etching process, SEM images of the substrate after removing the SU-8 and prior to the silicon dioxide deposition were also recorded (figure $4.8, \mathbf{C}$ ). Because here the pores are cylindrically shaped, the cone shape has to be a result of the silicon dioxide deposition process. Probably not only the surface of the substrate is coated during this process, but the silicon dioxide also accumulates inside the pores which leads to the shrinking of the radius. As the cone-shape of the pores is an artifact of the deposition process and the deviation from the desired nominal pore-size of $1 \mu \mathrm{m}$ is quite large, this approach was also discarded.

\subsubsection{Silicon nitride substrates}

The AFM and SEM images of the plain silicon substrates show a low surface roughness of $(1.58 \pm 0.34) \mathrm{nm}$ as well as cylindrically shaped pores (figure 4.8$)$. The surface roughness is here limited by the quality of the deposited material rather than the roughness of the silicon wafer itself, since its roughness is below $1 \mathrm{~nm}$ [115]. The roughness values obtained by AFM microscopy for the silicon nitride covered substrates show good concordance with literature values for PECVD silicon nitride deposited and examined by the same methods under the same conditions [116]. 
Electric lipid bilayer recordings can be performed at single channel resolution (chapter 7 ). In addition, impedance spectroscopy was used to show that highly insulating lipid bilayers can be formed on this type of substrate (section 6.2.3). Therefore, the plain silicon nitride substrates were used for all experiments.
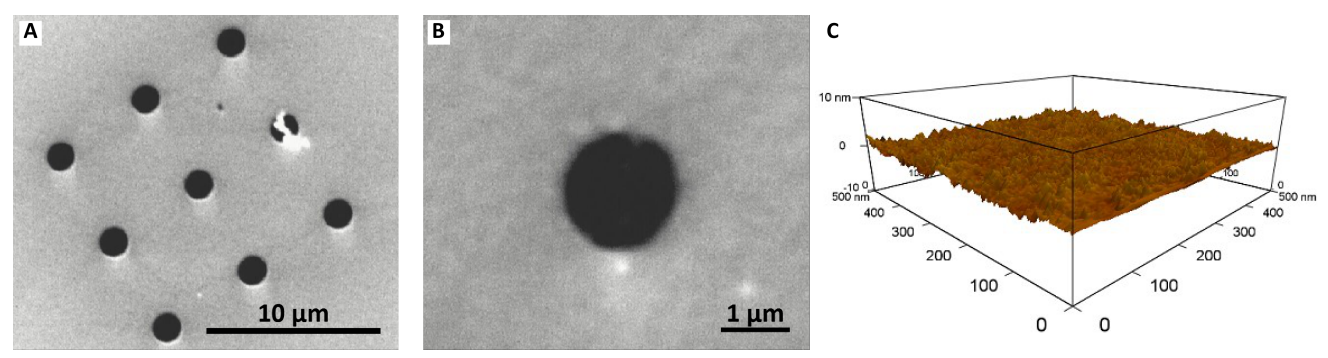

Figure 4.8: AFM and SEM images of the silicon nitride based substrate type with cylindrical pores $(\mathbf{A}, \mathbf{B})$ and low surface roughness $\mathbf{C}$ which was used in the experiments.

Passivation layers, which are mostly polymers or thermally grown oxides [117], are materials with a low dielectric constant that are deposited or grown in thick layers (hundreds of $\mathrm{nm}$ up to $\mu \mathrm{m}$ ). This procedure aims to reduce the capacitive noise of the substrates, which is important for single channel resolution. Other substrates are even completely fabricated in polymer material or glass [118]. The rms (root mean square) dielectric noise that is generated by the substrate can be described by equation 4.1 [119], where $D$ is the dielectric loss tangent of the materials, $f_{c}$ is the bandwidth of the experiment, $k$ the Boltzmann constant, $T$ the temperature and $C_{D}$ the capacitance of the substrate, which is dependent on the thickness $d$, the area $A$ and the dielectric constants of the material $\epsilon$ and in vacuum $\epsilon_{0}$, respectively (equation 4.2).

$$
\begin{gathered}
I_{D}\left(f_{c}\right)=\sqrt{\left(4 \pi k T D C_{d} c_{2} f_{c}^{2}\right)} \\
C_{D}=\epsilon \epsilon_{0} \frac{A}{d}
\end{gathered}
$$

However, it was possible to perform single-channel experiments without passivation layers as shown with alamethicin ion channels in chapter 7. 


\section{CHAPTER 5}

\section{Setup and sample chamber}

The setup used for the electric experiments was initially designed for a two-part project within the SFB 803 (Functionality controlled by organization in and between membranes). In this project, the mechanical stimulation of ion channels in the primary cilia, especially polycystin-2, is investigated in an in vivo and an in vitro approach. In the in vivo part primary cilia of MDCK cells were to be stimulated by attaching optically trapped beads to them and record the response of the ion channels in patch-clamp experiments. In the in vitro part, for which the microporous substrate in this work was designed, the expressed polycystin-2 ion channels were to be reconstituted in free-standing lipid bilayers and also stimulated by mechanical manipulation with optically trapped beads. The response of the ion channels were to be recorded by single channel lipid bilayer experiments. Therefore, the setup had to include an optical trap as well as the possibility of performing patch-clamp experiments and single channel lipid bilayer experiments. In addition, to visualize the lipid bilayer and the primary cilia, brightfield and fluorescence microscopy had to be implemented. All microscopy and optical trap related setup parts were built by Christopher Battle, the implementation of the low noise single channel recording and impedance spectroscopy setup was part of this work. Both parts of the setup are fully functional and the imaging of cilia and optical trap experiments as well as low noise single channel recordings and impedance spectroscopy is possible, but as the projects turned out to be quite ambitious, both did not use electrophysiology and the optical trap setup in combination. 


\subsection{Setup}

A photograph of the setup is shown in figure 5.1. The microscope body (1) as well as all electrophysiological setup parts such as the micromanipulator (8), the headstage for the single channel recordings, and the connection to the impedance spectrometer (7) are shown. The sample is mounted on the xyz-stage (5) and can be illuminated from below with an LED, or with a $473 \mathrm{~nm}$ diode laser, which is coupled into an optical fiber and then into the microscope body (3). The trapping laser is coupled into the microscope body at (4) and all associated optics are placed on the raised aluminum platform (6). The connections to the electrophysiological parts of the setup (patch-clamp amplifier and impedance spectrometer) are on the left side of the setup (7).

The microscope body, the headstage and the micromanipulator are incased in a faraday cage to shield the experiment from electromagnetic fields which is crucial for low noise recordings. The Faraday cage is made of $1 \mathrm{~mm}$ thick carbon steel plates with holes for the extending microscope parts like the camera and for any cables going out. The plates can be attached by magnets to four posts which can be easily rearranged on the optical table. The plates can be removed or replaced so that access to all sides of the setup is possible in case parts are added or have to be repositioned. The size of the faraday cage can also be modified, for example to exclude the lasers and therefore to further reduce the noise in the setup.

Another aspect for low noise electric recordings is to eliminate noise that comes from the power supplies of the equipment which has to be done by grounding rather than shielding. The Faraday cage is grounded to the optical table which is in turn grounded to earth. All lasers and the LED are mounted in a separate rack so that their power supplies do not interfere with the experiment. The noise contribution of all other power supplies, cables and electronic equipment was tested in a trial and error process and removed, grounded to a common point or separated from the setup. 


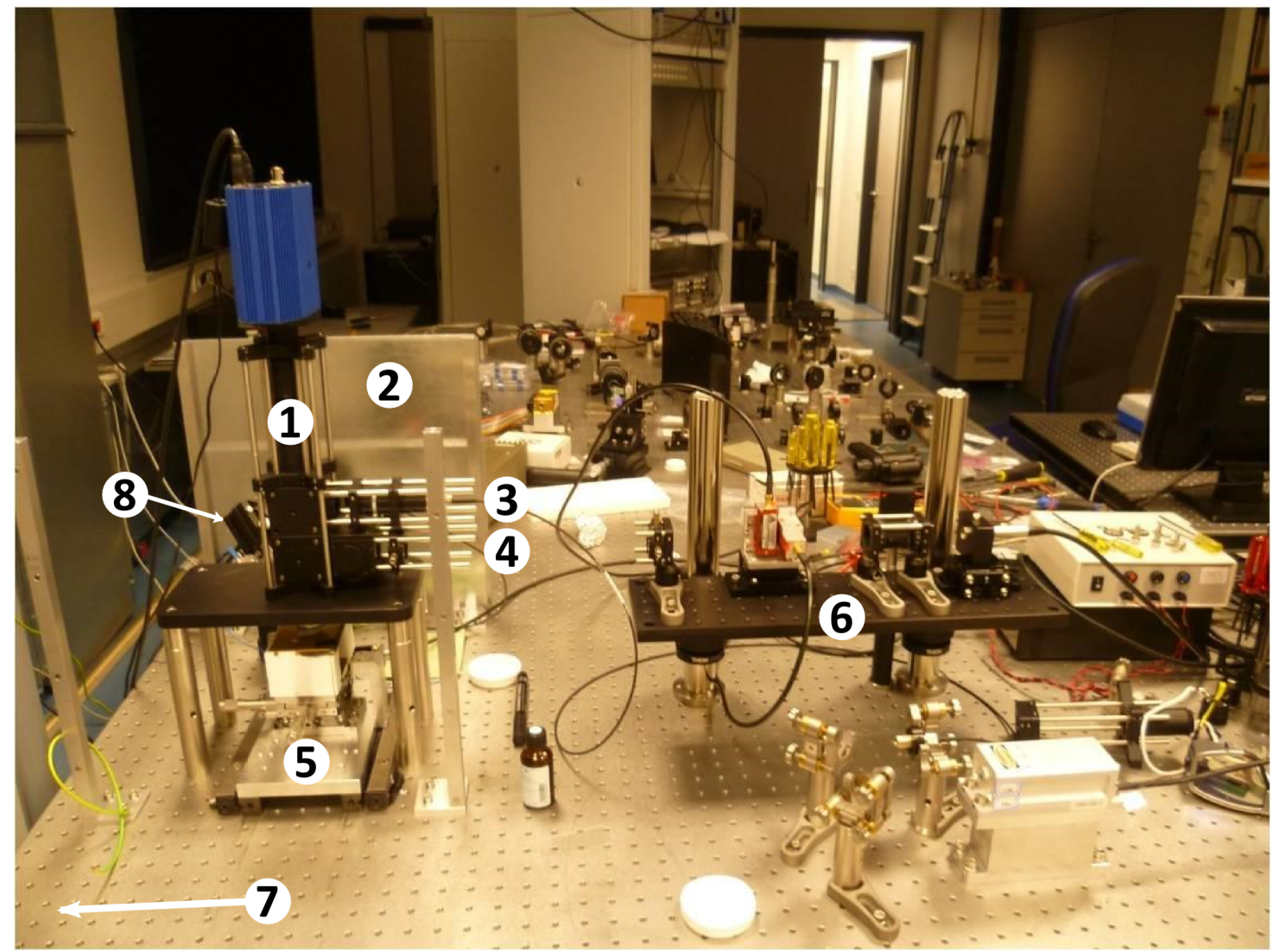

Figure 5.1: Photography (by Christopher Battle) of the setup including the microscope body $\mathbf{2}$, part of the faraday cage $(\mathbf{2})$ surrounding microscope and electrophysiology parts, lasers for optical trapping (4), and illumination (3). The sample can be illuminated from below with and LED placed under the xyz-stage (5). All associated optics for the optical trap are on an aluminum platform (6) and the connections to single channel amplifier and impedance spectrometer are on the left side (7). The micromanipulator for patch-clamp experiments is placed behind the microscope body (8).

The noise level of the whole setup, including substrate and sample chamber, was evaluated by mounting a substrate before the pore-etching procedure (with an unperforated silicon nitride layer) into the sample chamber, filling the electrolyte compartments with $1 \mathrm{M} \mathrm{KCl}$ solution (this concentration is also used for the single channel experiments) and recording the current response to a small applied potential. From these current traces, the rms (root mean square) value was calculated to $0.28 \mathrm{pA}$ at a cutoff frequency of $1 \mathrm{kHz}$. This value is low enough to rule out the possibility that small ion channel conductance levels cannot be resolved due to 
noise coming from the setup. The value increases to $2.4 \mathrm{pA}$ when lipid bilayers are prepared on the porous substrate (for a membrane resistance of $2 \mathrm{G} \Omega$ ). Since the noise is highly dependent on leak currents and therefore on the resistance of the lipid bilayers, it varies with different preparations. No difference was seen between integrated and external electrodes.

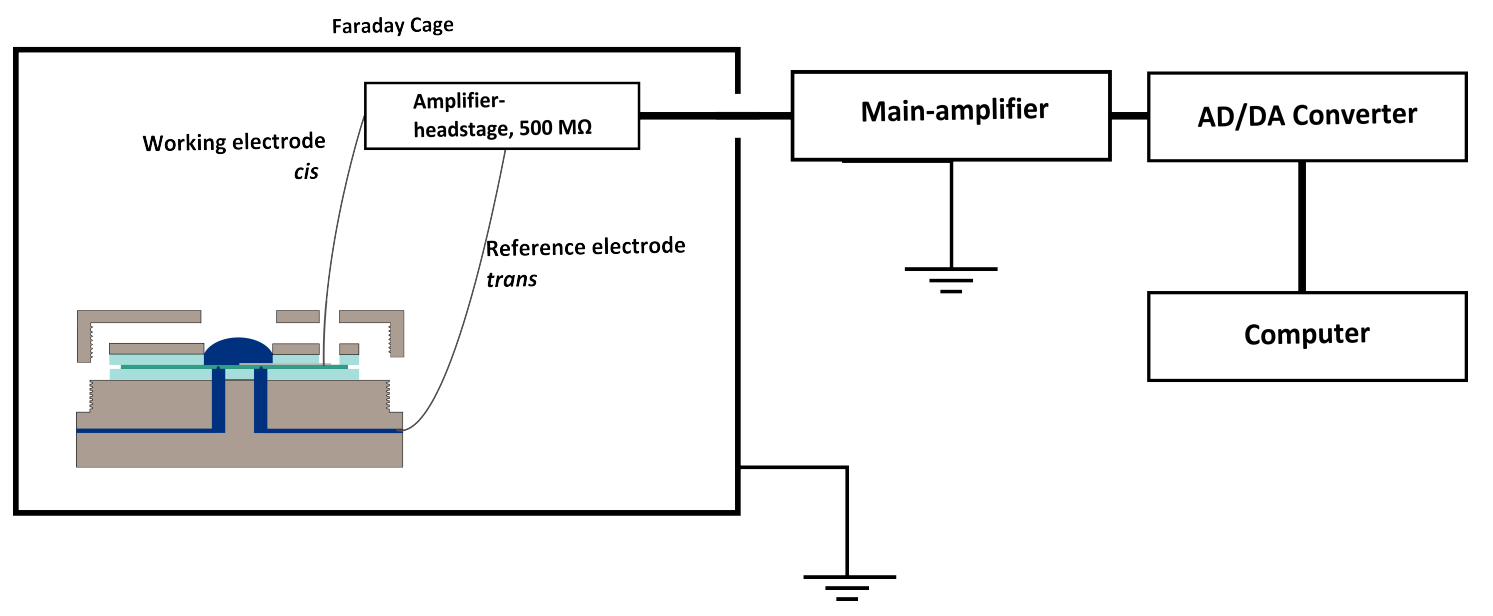

Figure 5.2: Setup for voltage clamp single channel recordings consisting of the sample chamber and the pre-amplifier that are enclosed in a Faraday cage, the main-amplifier, the $\mathrm{AD} / \mathrm{DA}$ converter and the computer with the acquisition software.

Figure 5.2 shows the electrophysiological parts of the setup. The sample chamber is described in more detail in section 5.2. The $\mathrm{Ag} / \mathrm{AgCl}$ electrodes were connected to a pre-amplifier with an access resistance of $500 \mathrm{M} \Omega$. The reference electrode, which determines the trans side and is kept at virtual ground, is the $\mathrm{Ag} / \mathrm{AgCl}$ wire in the bottom electrolye compartments, the working electrode is the integrated electrode on the top part and defines the cis side. The pre-amplifier is connected to the main amplifier (ELC-03XS, NPI Electronics, Tamm, GE), where the signal is amplified and filtered with a 4-pole bessel filter. The signals are digitized with a LIH8+8 AD/DA converter (HEKA Elektronik, Lambrecht, GE) and processed with the acquisition software (Patchmaster, HEKA Elektronik, Lambrecht, GE). Input signals are programmed with in the same software, converted to analog signals again by the LIH8 $+8 \mathrm{AD} / \mathrm{DA}$ converter which is connected to the signal input at the main amplifier. 


\subsection{Sample chamber}
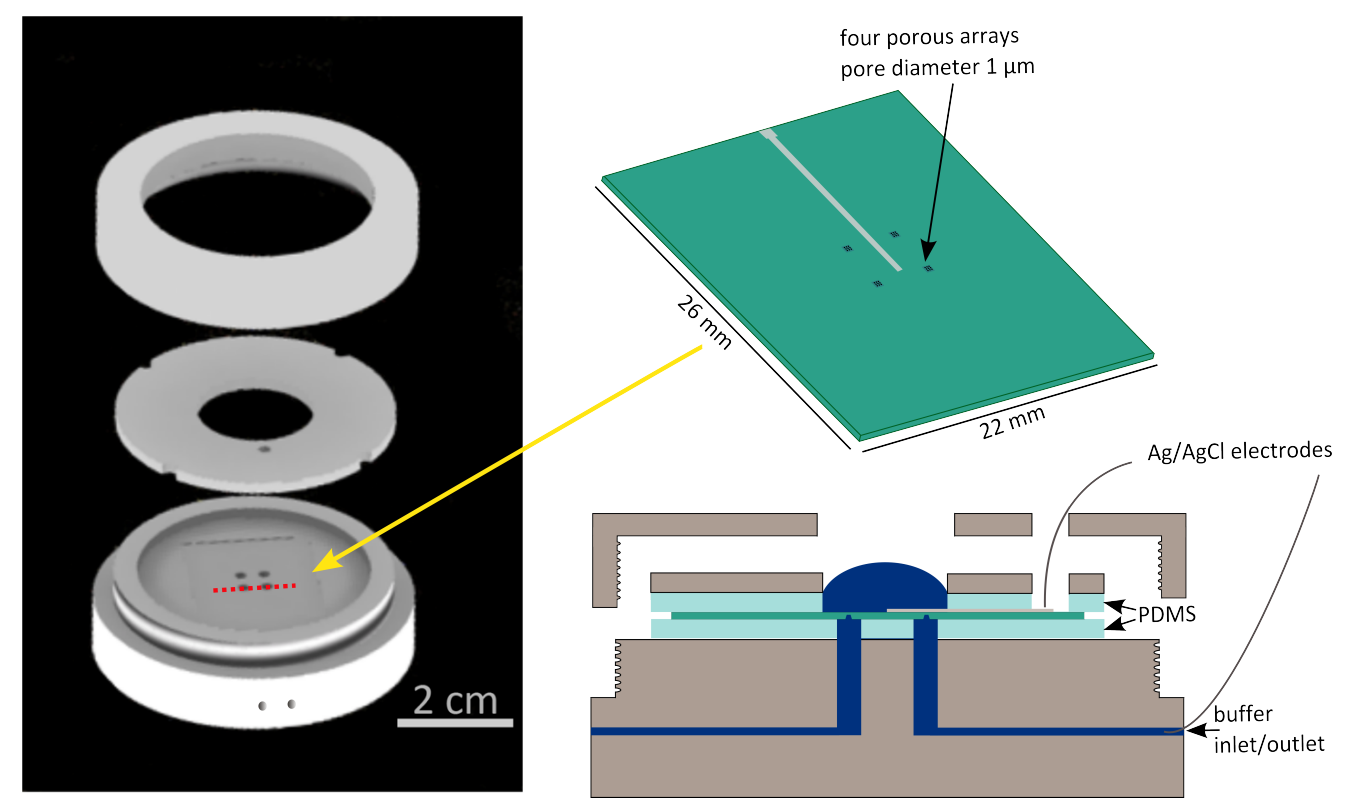

Figure 5.3: The sample chamber consists of three parts, with separated electrolyte compartments in the lower part for each of the arrays and a common compartment on the top. The chamber can be sealed tightly by a screw-cap.

The sample chamber had to meet several demands: It had to be suitable for electrical and optical experiments, which was achieved by adjusting the opening of the top compartment to the measurements of the microscope objectives. By including the integrated electrode on the top side of the microsubstrate, the access for the objective is facilitated.

The exchange of electrolyte solution or chemicals during the experiment must be possible individually for each of the porous areas, so in- and outlets for each of the lower electrolyte compartments were incorporated that can be connected to tubing. The solutions can then be exchanged using syringes.

To be able to perform four electrical experiments on one substrate it is necessary to electrically separate the four areas, so there are four separate buffer compartments on the lower side, whereas the top compartment can be the same for all arrays. Figure 5.3 shows the sample chamber that was designed for that purpose. It consists of three parts, the lowest part contains separated buffer compartments as 
well as the in- and outlets for each of the four pore areas. As a material for the sample chamber PMMA was chosen, because is biocompatible and does not interfere with electric or fluorescence microscopy experiments. The substrate is placed between the lower part and the middle plate, and the chamber can be screwed tightly together by the lid. To prevent breaking of the substrate, two layers of PDMS (poly-dimethyl siloxane, Sylgard 184 Elastomer Kit, Dow Corning, US), a compressible, rubberlike material, are used, which are placed directly on both sides of the microsubstrate and form a tight seal, thereby separating top and bottom of the substrate completely.

\subsection{Lipid bilayer formation}

\subsubsection{Preparation of Giant Unilamellar Vesicles (GUVs)}

For preparing vesicles with a diameter of $10-60 \mu \mathrm{m}$, the electroswelling method, developed in 1986 by Angelova and Dimitrov [120, 121, 122], was used. Giant Unilamellar Vesicles (GUVs) were used for the preparation of pore-spanning lipid bilayers, because one vesicle can cover the whole porous array.

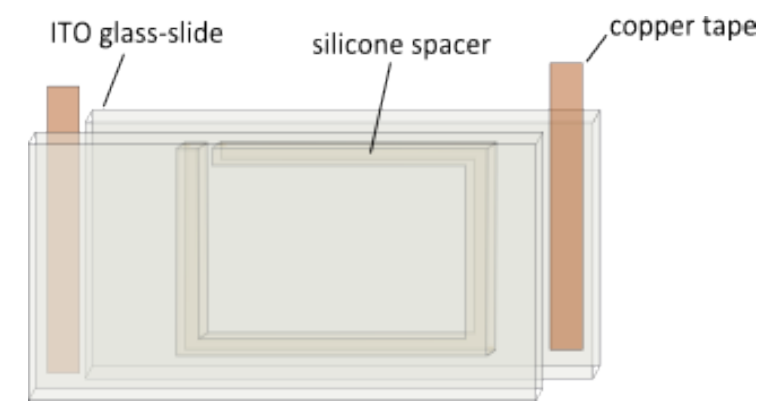

Figure 5.4: The chamber for the electroformation of GUVs consists of two ITO-coated glass slides separated by a silicone spacer, and copper tape for the electrical connection.

The desired mixture of lipids was dissolved in chloroform to a concentration of $1 \mathrm{mg} / \mathrm{ml}$. The lipid solution was pipetted drop wise on indium-tin-oxide (ITO)coated glass slides (25 $\mu \mathrm{l}$ each, Präzisionsglas \& Optik GmbH, Isernloh, GE) and the solvent was removed in a desiccator overnight. For the electroswelling procedure a chamber was formed of two glass slides and a silicone spacer (figure 5.4) 
of $1 \mathrm{~mm}$ thickness so that the chamber volume is approximately $1 \mathrm{ml}$. The chamber was filled with $500 \mathrm{mM}$ D-Sorbitol solution and copper tape with conducting adhesive (RS Components, GE) was fixed as an electrical contact on each of the two glass slides. A sine-wave voltage with an amplitude of $3 \mathrm{~V}$ and a frequency of $5 \mathrm{~Hz}$ was applied for $2 \mathrm{~h}$, during which the GUVs formed. The vesicles were then removed from the chamber and either used immediately or stored at $4{ }^{\circ} \mathrm{C}$ for up to one week.

Lipid mixtures used were: pure DOPC for impedance spectroscopy and alamethicin single channel recordings and DOPC doped with 0.5 mol\% Oregon-Green 488 DHPE (Oregon Green 488 1,2-Dihexadecanoyl-sn-Glycero-3-Phosphoethanolamine, Life Technologies, Paisley, UK) for imaging the lipid bilayers on unfunctionalized substrates and DOPC doped with 1 mol\% $\beta$-BODIPY-500/510-C 12 -HPE (Life Technologies, Paisley, UK) for imaging the lipid bilayers on gold-functionalized substrates. Lipids were obtained from Avanti Polar Lipids (Alabaster, USA), alamethicin from Sigma Aldrich (Taufkirchen, GE).

\subsubsection{Preparation of lipid bilayers and alamethicin reconstitution}

The substrates were mounted in the sample chamber as shown in figure 5.3. GUV solution $(20 \mu \mathrm{l})$ was pipetted onto each of the porous arrays and a slight suction was applied with a syringe on the tubing on the lower electrolyte compartment so that the vesicles were drawn towards the pores. After 30 min of incubation the top compartment was washed and filled with $300 \mu$ l electrolyte solution $(1 \mathrm{M} \mathrm{KCl,} 10$ mM MES, $10 \mathrm{mM}$ MES, $0.06 \mathrm{mM}$ EDTA, pH 7.0). This procedure was repeated until a membrane was formed.

For the single channel experiments, alamethicin was added to the top side of the chamber from a stock solution of $0.05 \mathrm{mg} / \mathrm{ml}$ in ethanol to a final concentration of $127 \mu \mathrm{M}$ after lipid bilayer formation was confirmed by impedance spectroscopy. After 5 min a holding potential was applied and, if single channel events were observed, the cis side was rinsed with electrolyte solution to remove the remaining ethanol. 


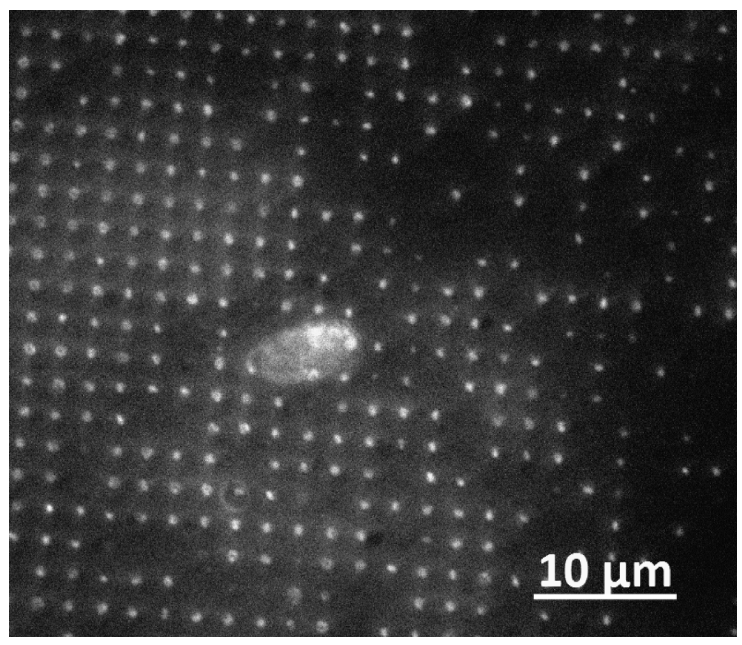

Figure 5.5: Lipid bilayers on unfunctionalized silicon nitride. The lipid bilayer was prepared from DOPC vesicles doped with 0.5 mol\% Oregon-Green 488 DHPE.

The lipid bilayers were prepared on the bare silicon nitride in all impedance and single-channel experiments after thorough cleaning of the substrates in $\mathrm{H}_{2} \mathrm{O} / \mathrm{H}_{2} \mathrm{O}_{2} / \mathrm{NH}_{3}$ at $70^{\circ} \mathrm{C}$ for $15 \mathrm{~min}$ and $\mathrm{O}_{2}$ plasma treatment for $10 \mathrm{~min}$. The pore-spanning lipid bilayers were visualized by fluorescence microscopy for 900-pore substrates as shown in figure 5.5. Impedance spectroscopy was used to monitor lipid bilayer formation before each experiment for the 9-pore substrates as shown in section 6.2.3.
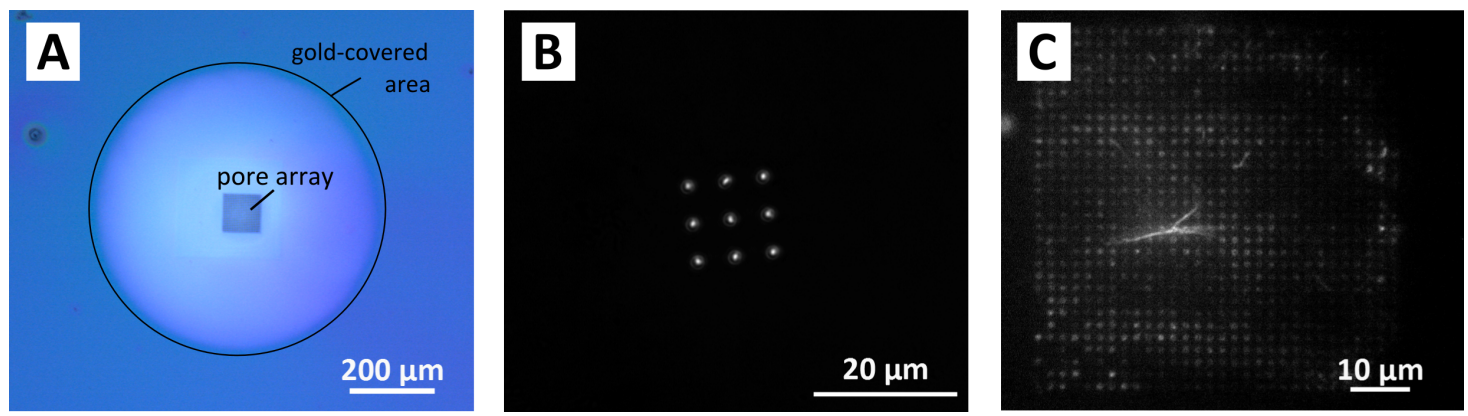

Figure 5.6: Lipid bilayers on gold/thiol functionalized substrates. (A) was taken with a 100x water-dipping objective (LUMPlanFL W, Olympus Corp., Tokyo, JP), (B) with a $63 \mathrm{x}$ water immersion objective (C-Apochromat, Carl Zeiss AG, Jena, GE ). Both are DOPC lipid bilayers doped with 1 mol\% $\beta$-BODIPY-500/510- ${ }_{12}$-HPE.

We also tried to prepare lipid bilayers on functionalized substrates. For functionalization titanium $(10 \mathrm{~nm})$ and gold $(100 \mathrm{~nm})$ were deposited on the substrate 
by e-beam and thermal evaporation respectively. Custom-built mechanical masks were used to deposit the metals in a circle with a radius of $300 \mu \mathrm{m}$ on the porous array as shown in figure 5.6, A. The gold was functionalized via thiol-gold chemistry by incubation in a $0.1 \mathrm{mM}$ solution of the cholesterol-derivative CPEO3 (Cholesterylpolyethylenoxythiol, provided by Prof. Claudia Steinem) in 1-propanol at $4^{\circ} \mathrm{C}$ overnight.

With this functionalization, lipid bilayers were obtained on substrates with 900 and 9 pores (figure $5.6 \mathbf{B}$ and $\mathbf{C}$ ), although also in the fluorescence images of the bilayer-covered 900-pore substrates the incomplete coverage of the pore-array can be observed.

Because lipid bilayer formation was equally often achieved with functionalized and unfunctionalized substrates and the preparation on the unfunctionalized substrates requires less fabrication steps, all lipid bilayers used in electric experiments were performed with lipid bilayers on plain silicon nitride as described above.

The preparation of lipid bilayers on plain silicon nitride has been done before and also high resistances in electrical measurements have been obtained [44], but also problems with this kind of preparation, for example the fast rupturing of the lipid bilayer, have been reported [123]. In this work, however, once a lipid bilayer was prepared, it was stable for several hours, but a functionalization could be reconsidered if such problems occur in future experiments, when other types of lipids or proteins are used. 



\section{CHAPTER 6}

\section{Impedance Spectroscopy}

The use of impedance spectroscopy as a tool for the investigation of the behavior of electrically complex systems emerged in the early 1980s [124] and developed into a standard method for electrochemical research. It is used in the development of fuel cells [125], solar cells [126] and to study corrosion processes [127] and has also become a valuable tool for biosensor applications, because of its sensitivity to processes occurring at electrode surfaces. For example, immobilization of antibodies on thin insulating surfaces are usually used for impedance spectroscopy in immunosensing devices. The decrease of the capacitance that comes with binding of components (proteins, bacteria, viruses) to the antibody and the subsequent increase of thickness can be detected (equation 6.8) [128, 129]. Alternatively, the increase of resistance at the electrode-solution interface can be detected. Binding studies of enzymes, DNA and even whole cells can be investigated by impedance spectroscopy [130]. Other applications are cell counting [131, 132] and cytotoxicity studies [133, 134].

In lipid bilayer research, for example, the detailed investigation of lipid bilayer formation or the protein related change of the ion permeability of lipid bilayers $[135,136]$ can be examined.

When performing electrophysiological experiments with lipid bilayers and proteins, not only the electrical characteristics of the investigated components play a role in the response to electric stimulation, but also of the supporting substrate. In case of a lipid bilayer on a porous silicon nitride layer, the major contribution to the total capacitance comes from the underlying substrate and only a small part from the membrane, whereas the high giga-ohm resistance is a result of the membrane 
formation. In order to accurately analyze the results obtained in an experiment, the knowledge of the electrical behavior of the substrate is important.

Impedance spectroscopy is a valuable tool for a detailed analysis of the electrical components of a system. Because changes in the substrate fabrication, like for example inhomogeneous coating or small defects in the silicon nitride layer, lead to a change in electrical response, it is also very useful to determine the quality of a substrate to ensure reproducibility.

In this work, impedance spectroscopy was used to electrically analyze the substrate and the differences between the integrated and external electrodes. Also membrane formation was monitored.

\subsection{Theoretical Background}

The impedance is the frequency-dependent resistance of a system. The principle of impedance spectroscopy is to apply an AC voltage with a small amplitude to a system and record its response. Just like Ohm's law describes the resistance in the frequency-independent case, the impedance can be described as the ratio between voltage and current, both being dependent on the frequency $\omega$ and the time $t$.

$$
Z(\omega)=\frac{U(t, \omega)}{I(t, \omega)}
$$

The excitation signal, an alternating voltage with the amplitude $U_{0}$ is

$$
U_{t}=U_{0} e^{i(\omega t)}
$$

the current response in a linear system is phase-shifted and has the amplitude $I_{0}$.

$$
I_{t}=I_{0} e^{i(\omega t+\phi)}
$$

The impedance is the ratio of these signals and can also be described as a complex number.

$$
Z(\omega)=\frac{U_{0}}{I_{0}} e^{i \phi(\omega)}=|Z| e^{i \phi(\omega)}
$$

Using Euler's relation $\exp (\mathrm{i} \phi)=\cos (\phi)$-isin $(\phi)$, the impedance can be split into a 
real part, $Z_{r e}$, and an imaginary part, $Z_{i m}$. The real and imaginary parts $Z_{r e}(\omega)$ and $Z_{i m}(\omega)$ are then:

$$
Z_{r e}(\omega)=\frac{U_{0}}{I_{0}} \cos (\phi(\omega)) \text { and } Z_{i m}(\omega)=-\frac{U_{0}}{I_{0}} \sin (\phi(\omega))
$$

The impedance $Z(\omega)$ is, according to equation 6.6, in Cartesian and polar coordinates, respectively:

$$
Z(\omega)=Z_{r e}(\omega)+i Z_{i m}(\omega) \text { and } Z(\omega)=|Z|(\omega) e^{i \phi(\omega)}
$$

The relation of $Z_{r e}(\omega), Z_{i m}(\omega)$ and the phase angle $\phi(\omega)$ can be illustrated in a vector diagram (figure 6.1). Here, the modulus $|Z|(\omega)$ is the vector between the imaginary and the real part of the impedance. The phase angle is the angle between the real axis and the modulus $|Z|(\omega)$. The relation of these parameters are:

$$
|Z|(\omega)=\sqrt{Z_{r e}(\omega)^{2}+Z_{i m}(\omega)^{2}} \text { and } \phi=\arctan \frac{Z_{i m}}{Z_{r e}}
$$

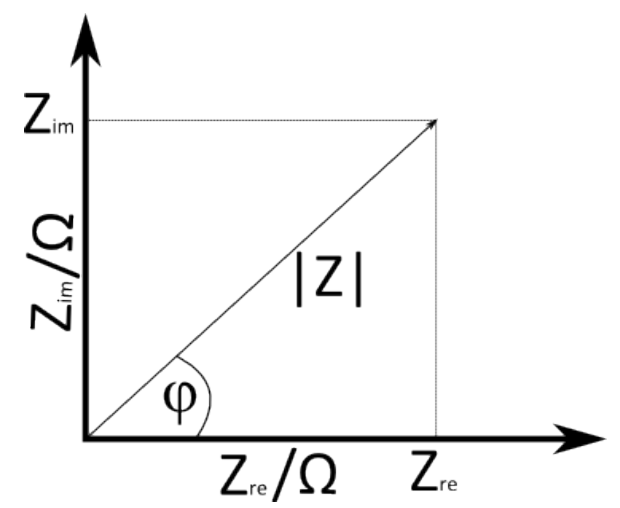

Figure 6.1: In a vector diagram the modulus $|Z|(\omega)$ of the impedance is the vector between the imaginary and the real part of the impedance. The phase angle is the the angle between absolute value and real part of the impedance.

Impedance spectra are usually displayed in two types of plots: Nyquist plots and Bode plots. In Nyquist plots (figure 6.2, A) the imaginary part is plotted over the real part of the impedance so that each point in the plot depicts $Z_{r e}(\omega)$ and $Z_{i m}(\omega)$ at a specific frequency or a vector of length $|Z|(\omega)$ from the origin (cp. figure 6.1). Nyquist plots provide an easy overview of different circuit elements, because of 
their characteristic appearance. An example of a simple $\mathrm{R}(\mathrm{RC})$ circuit (a resistor in series with a second resistor and a capacitance in parallel, figure $6.2 \mathbf{C}$ ) was drawn in figure $6.2, \mathrm{~B}$, which appears as a semi-circle. A second RC element in series to the first one would appear as a second semi-circle in the spectrum.

Bode plots show $\log |Z|(\omega)$ and the phase angle $\phi(\omega)$ respectively plotted over $\log (\mathrm{f})$ and provide direct frequency information. An example for the same $\mathrm{R}(\mathrm{RC})$ circuit used for the Nyquist plot is shown in figure 6.2, C [137, 138].
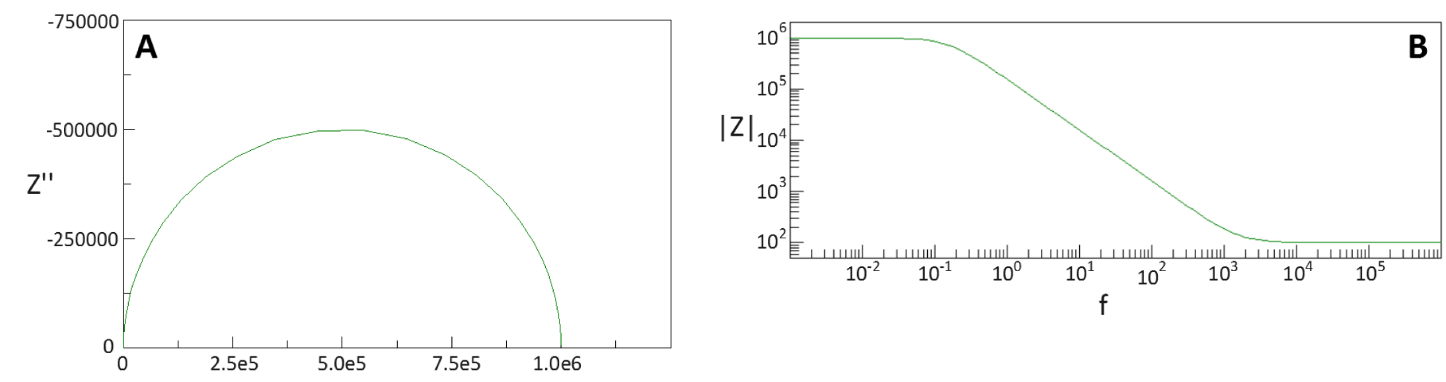

C
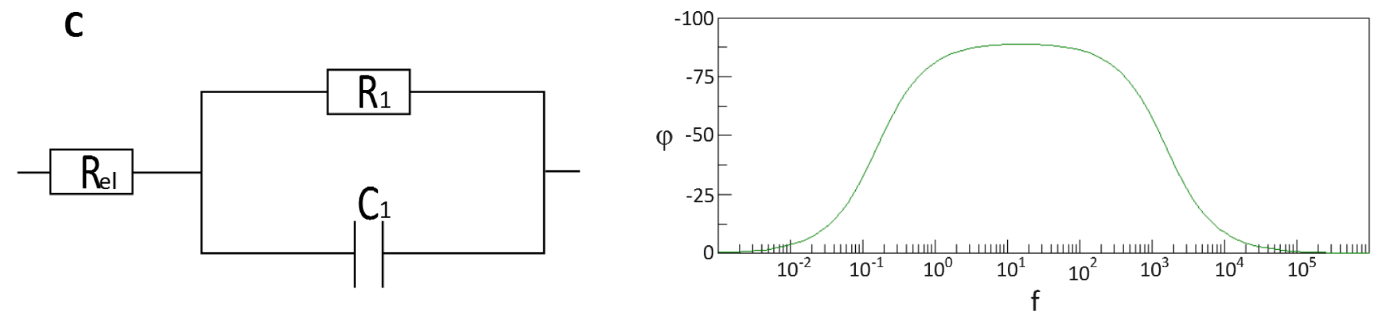

Figure 6.2: The figure shows Nyquist $\mathbf{A}$ and Bode plots $\mathbf{B}$ of an $R_{e l}\left(R_{1} \mathrm{C}_{1}\right)$ circuit

C. The parameters for the circuit elements are: $R_{e l}=100 \Omega, R_{1}=10^{6} \Omega$ and

$$
C_{1}=10^{-6} \mathrm{~F}
$$

The current response to a voltage stimulus is not linear in a complex electric circuit. Non-linear responses contain harmonics of the input frequency which would make the analysis very difficult. Therefore, only small amplitudes $(1-10 \mathrm{mV})$ are applied so that a pseudo-linearity between input voltage and current output is created and the analysis is considerably facilitated (figure 6.3). 


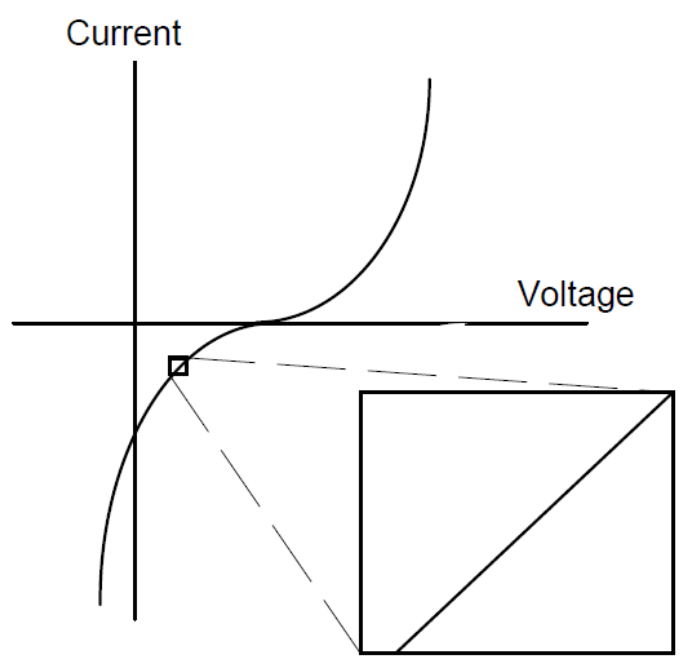

Figure 6.3: A pseudo-linear current-voltage relation is created by using only a small voltage amplitude [138].

\subsubsection{Equivalent circuit elements}

The most accurate way of analyzing impedance spectra is to derive the parameters using a continuum-mechanics approach. Here, a mathematical model is set up with appropriate boundary conditions for the specific system. From the resulting equation for the impedance, a physical model can then be derived.

The data analysis of impedance spectra can also be done by fitting the effective impedance corresponding to a network of circuit elements such as resistors, capacitors or inductors that resemble the different electric responses of the specimen. This approach is very intuitive and gives a fast overview of the circuit elements for a system. Analysis software usually use a non-linear least square fitting algorithm and an iterative process, which requires an appropriate starting value for each circuit component. These starting values can be approximated by comparison with similar, already characterized systems, or by simulating parts of the spectrum individually. This approach is therefore most suitable for systems that are already characterized or contain clearly recognizable circuit elements.

This semi-empirical approach can provide easy access to the most relevant parameters of a system [125], but there is a risk of using an overcomplicated circuit when a simpler one might fit equally well. 
In this work, the semi-empirical approach was used for the estimation of the relevant parameters of the microporous substrates as well as the lipid bilayer. Silicon/silicon nitride based systems are well-known and many equivalent models are available in the literature so that a good estimation of the circuit elements involved was possible.

\section{Ohmic resistance and capacitance}

In the analysis of impedance spectra of lipid bilayers the ohmic resistance in parallel to a capacitance is used to describe the lipid bilayer. Because of its hydrophobic core that is not permeable for charge carriers, the lipid bilayer is responsible for the high ohmic resistance that can be observed in all membrane-related electric experiments. Additionally, the lipid bilayer between the two electrolyte compartments acts as a capacitor (figure 6.4).

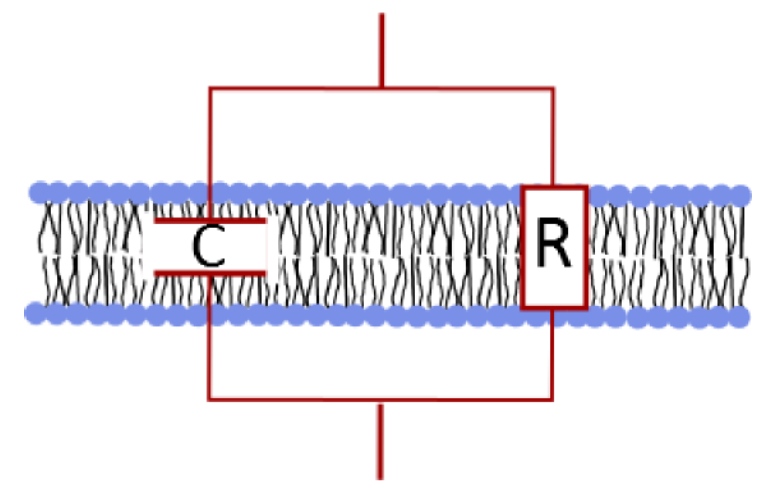

Figure 6.4: The lipid bilayer can be modeled by a resistance and a capacitor in parallel. The hydrophobic core acts as a resistor and as a dielectric layer between the two electrolyte compartments.

As already pointed out above, the ohmic resistance is the frequency-independent impedance of a system. Here, the phase angle is $0^{\circ}$.

The capacitance of a planar lipid bilayer can be regarded as a parallel plate capacitance, where the lipid bilayer acts as a dielectric layer between the two conducting electrolyte compartments. The capacitance of a parallel plate capacitor is dependent on the thickness of the dielectric layer $d$ and the area $A$. Also the dielectric constant $\epsilon_{r}$, a material constant for the dielectric layer, and the dielectric constant in vacuum $\epsilon_{0}$ have to be taken into account. 


$$
C=\epsilon_{0} \epsilon_{r} \frac{A}{d}
$$

The thickness of lipid bilayers is in the range of 4-6 $\mathrm{nm}$, depending on the length of the lipid side chains. For the dielectric constant $\epsilon_{r}$, the value for the dielectric constant of the hydrocarbon chains is about 2.6, according to S. Ohki [139], but the values can be higher for specific lipids; e.g. for DPPE bilayers the dielectric constant was estimated to 3.3 by electrostatic force spectroscopy [140]. The hydrophobic headgroups exhibit much larger dielectric constants in the order of 10-80 (for comparison: 78 for distilled water at $25^{\circ} \mathrm{C}$ [141]) for a headgroup thickness of 0.6-1.0 nm according to [124]. From these values, the capacity of lipid bilayers was estimated. For lipid bilayers prepared by the Mueller-Rudin and Montal-Mueller techniques (chapter 2.2) the capacity was found to be $0.5-1.0 \mu \mathrm{F} / \mathrm{cm}^{2}$ [142]. The impedance of a capacitor is given in equation 6.9 and the phase angle for a capacitor in an electric circuit is $-90^{\circ}$.

$$
(\omega)=\frac{1}{i \omega C}
$$

\section{The Constant Phase Element (CPE)}

The Constant Phase Element (CPE) was developed by Cole and Cole in the 1940s [143] and takes non-ideal capacitive behavior into account. In the Nyquist plot a $\mathrm{CPE}$ in parallel with a resistor appears as a depressed semi-circle with its center below the $\mathrm{x}$-axis (figure 6.5). Its phase angle is larger than $-90^{\circ}$. 

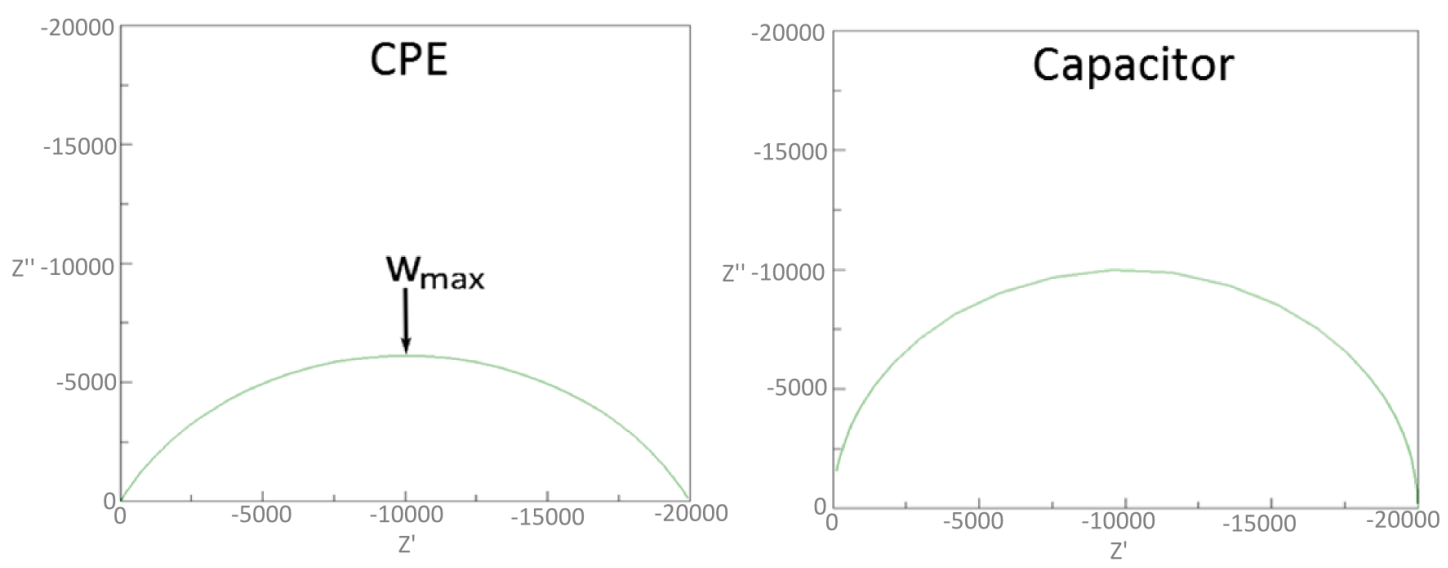

Figure 6.5: In a Bode plot, the CPE element in parallel to a resistor appears as a depressed semi circle compared to an ideal capacitance.

The impedance of the CPE element is given by equation 6.10 .

$$
Z_{C P E}=\frac{1}{(i \omega)^{P} T}
$$

The exponent $P$ is $0<\mathrm{P}<1$ and gives an estimate of the deviation of the phase angle from the ideal capacitive phase angle by $-(90 \cdot \mathrm{P})$.

For an RC circuit the "true" capacitance can be calculated from the parameter $C P E-T$, if the frequency at the highest point of the semicircle $\left(\omega_{\max }\right)$ is known, as proposed by C.S. Hsu and F. Mansfield [144]:

$$
C=\left(\omega_{\max }\right)^{P-1} C P E-T
$$

Reasons for the non-ideal capacitive behavior can be a distribution of time constants along the surface is considered [145], caused by inhomogeneities in the system, such as porous electrodes, variations in the thickness of or defects in surface coatings. In these cases, the implementation of a CPE instead of an ideal capacitor can fit the data more accurately. A CPE can also indicate diffusive processes. If the exponent $P$ has a value of 0.5 , the CPE resembles a Warburg impedance [146]. 


\section{The Warburg Impedance}

The Warburg impedance describes diffusion-related transport processes in an electrochemical system and was mentioned for the first time in 1899 by E. Warburg [147], who proposed a polarization resistance in series with a polarization capacitance for the description of electrochemical interfaces. J. E. B. Randles [148] used an equivalent circuit consisting of a charge transfer resistance in series with the Warburg resistance and in parallel to a capacitance to describe the frequency dependent behavior of redox reactions at a metal electrode-electrolyte interface. This model, namely the Randle circuit (figure 6.6), is used for the description of most diffusion processes in impedance spectroscopy.

When an electrochemical reaction takes place at the interface between electrolyte and electrode, a faradaic current, a charge transfer resistance, arises due to the electron transfer across the interface (figure 6.6, $R_{1}$ ). A non-faradaic component is the result of the charge separation at the interface (figure 6.6, $C_{1}$ ). The Warburg impedance in series with the charge transfer resistance accounts for the impedance due to mass transfer and it is frequency dependent.

The impedance of the Warburg element is given in equation 6.12.

$$
Z(\omega)=\sigma(1-i) \omega^{-0.5}
$$

It is valid for diffusion layers of infinite thickness, which means that the distance covered by the charge carriers is small compared to the thickness of the diffusion layer. If this is not the case, the thickness of the diffusion layer $\delta$ has to be taken into account:

$$
Z(\omega)=\sigma(1-i) \omega^{-0.5} \tanh \left(\delta\left(\frac{i \omega}{D}\right)^{0.5}\right)
$$

The Warburg constant $\sigma$ is given by equation 6.14 , where the diffusion coefficients $D_{\text {red }}$ and $D_{o x}$ can be determined. Here, $F$ is the Faraday constant, $n$ the number of exchanged electrons, $T$ the temperature, $R$ the gas constant and $A$ the surface area of the electrode [138].

$$
\sigma=\frac{R T}{\sqrt{2} n^{2} F^{2} A}\left(\frac{1}{c_{o x} \sqrt{D_{o x}}}\right)\left(\frac{1}{c_{r e d} \sqrt{D_{\text {red }}}}\right)
$$

In a Bode plot, the Warburg impedance can be observed as a diagonal line with a 
slope of 0.5 and the Nyquist plot shows a phase shift of $45^{\circ}$. It is most prominent at low frequencies, because here the ions have time to diffuse further than at high frequencies.

The Warburg impedance has not only been used for the description of electrodes, but it is also of relevance for the interpretation of the electric behavior of biological samples. Diffusion of charge carriers also occurs, for example, through ion channels and was observed in studies of Gramicidin-D [45].

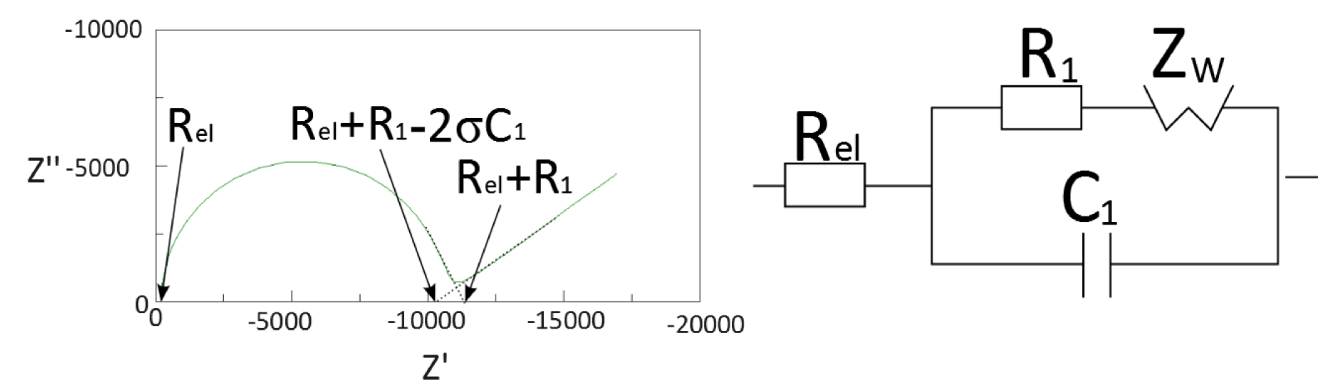

Figure 6.6: In a Bode plot the Warburg element appears as streight line at an angle of $45^{\circ}$.

\subsection{Results and discussion of impedance spectroscopy experiments}

In impedance spectroscopy experiments of lipid bilayers, it is important to first characterize the underlying substrate, because the spectra show not only the electric behavior of the membrane itself, but the response of the whole assembly. For this reason, the substrates were characterized in respect to their resistive and capacitive behavior and the influence of the integrated electrode was extracted.

Before each experiment, an impedance spectrum of the uncovered porous substrate was recorded to verify that all pores in the array under investigation were completely open and filled with electrolyte solution. This way, preparation related errors such as insufficient sealing of the chamber, leading to a merging of the lower electrolyte compartments, were avoided. 


\subsubsection{Electical models for silicon/silicon nitride based porous substrates}

The impedance spectra were analyzed with the ZView software (Scribner Associates, Inc, Southern Pines, US) that uses pre-defined circuit models with initial values for each element of the circuit which are then adjusted to the spectrum in an iterative process as described in section 6.1.1. This requires knowledge about a reasonable circuit model before the analysing process. A good starting point for the consideration of which model to use for the analysis is to review already existing models for similar systems.

Silicon nitride based porous substrates similar to those used in this work were already used and characterized. Although all systems differ only in the thickness of the silicon nitride layer, the pore-size, pore-number, the type of electrodes and the application of passivation layers such as SU-8, the proposed equivalent circuits are quite different. Kresak et al. [44] use a substrate that contains pores (diameters of $50-200 \mathrm{~nm}$ ) etched into a $100 \mathrm{~nm}$ thick silicon nitride layer. They describe the influence of the different parts of the substrate in great detail. For fitting, they use the circuit shown in figure $6.7, \mathbf{A}$. Here, $R_{e l}$ is the resistance of the electrolyte solution, $R_{S i N}$ and $C_{S i N}$ are the resistance and capacitance of the thin silicon nitride layer and $R_{S i}$ and $C_{S i}$ account for the rest of the substrate, including the silicon nitride on the bulk silicon. They observe two dispersions accounting for two different time constants in the spectra both with and without lipid bilayer. 


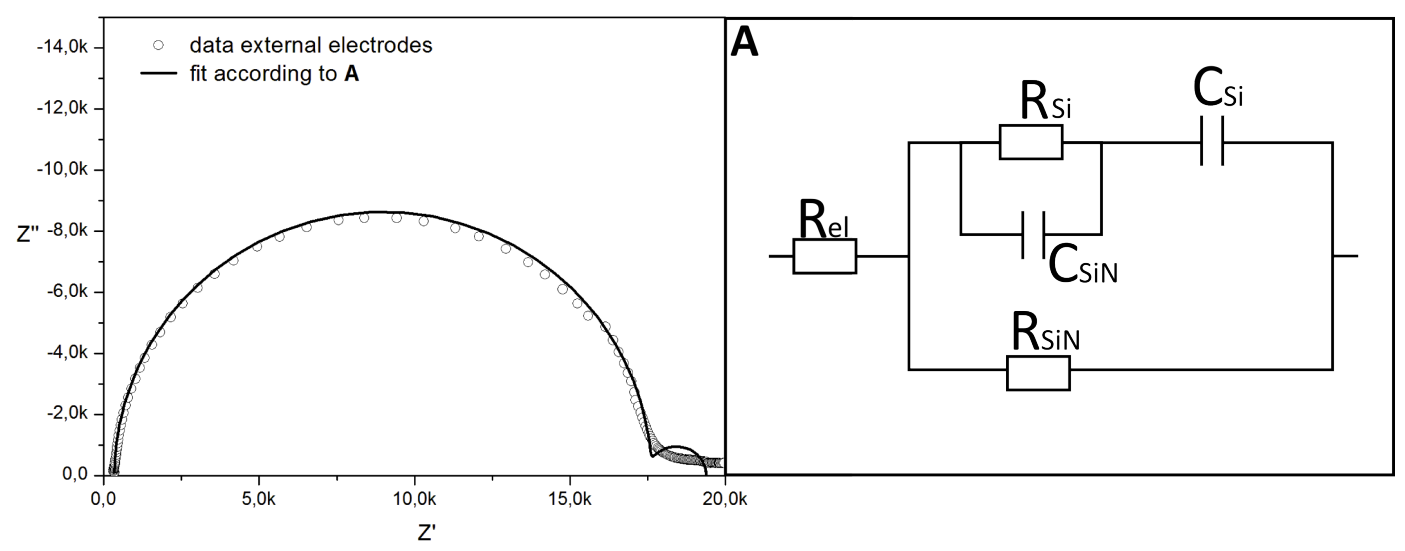

Figure 6.7: Impedance spectra fitted with the equivalent circuit proposed by Kresak et al. (A), impedance data are displayed as white circles and the fit as a solid line.

Another model, an $\mathrm{R}(\mathrm{RC})(\mathrm{RC})$ circuit, was proposed in a publication by the group of L. Tiefenauer [42] for silicon substrates with a $300 \mathrm{~nm}$ thick silicon nitride layer containing more than $10^{4}$ pores with a diameter of $200 \mathrm{~nm}$ and $800 \mathrm{~nm}$ respectively, per array. V. B. Sundaresan et al. also proposed a model that uses one RC element to describe the porous silicon nitride layer and multiple $\mathrm{RC}$ elements for the pyramidal opening, which can be reduced to one additional RC element [149]. This circuit is displayed in figure $6.8, \mathrm{~B}$. Again, $R_{e l}$ accounts for the electrolyte resistance, $R_{S i N}$ and $\mathrm{C}_{S i N}$ for the porous silicon nitride membrane and $R_{S i}$ and $C_{S i}$ for the bulk silicon. The spectra obtained by these groups also show two dispersions, one in the high frequency part and one in the low frequency part. 


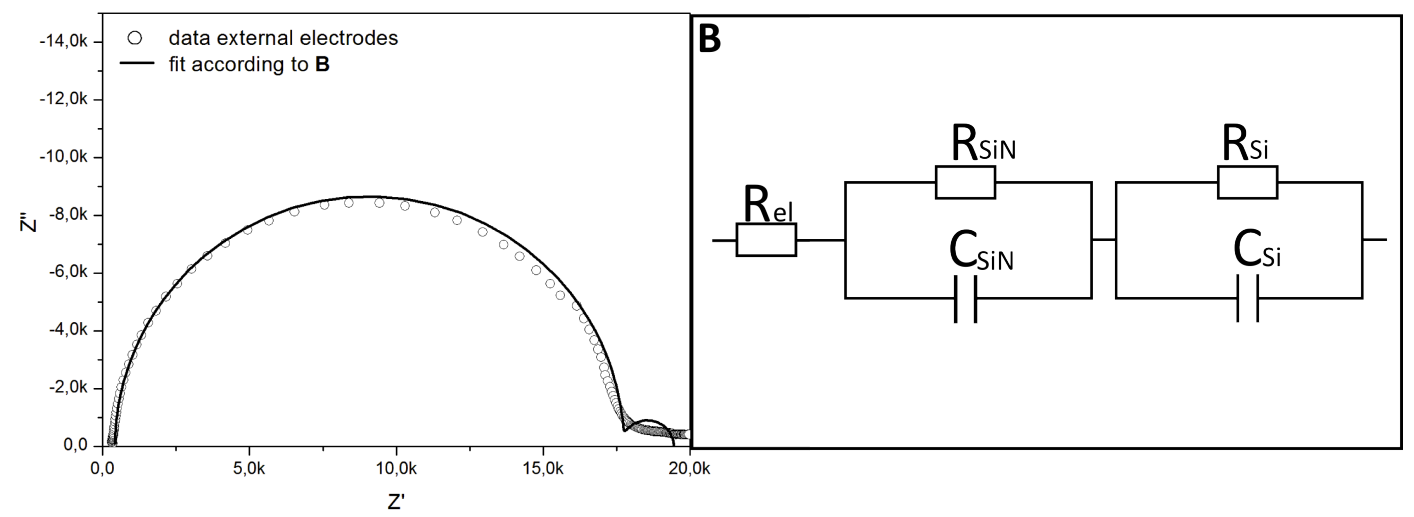

Figure 6.8: Impedance spectra fitted with the equivalent circuit proposed by Tiefenauer et al. (B), impedance data are displayed as white circles and the fit as a solid line.

The spectra of the substrates recorded in this work show only one distinct capacitance and an ohmic resistance in the high frequency part. In the lower frequency part, a very small second dispersion can be observed. When the spectra obtained in this work are fitted with the equivalent circuits shown in figure 6.7, $\mathbf{A}$ and figure $6.8, \mathrm{~B}, R_{S i N}$ and $C_{S i N}$ at the high frequency part can be fitted well, whereas the second part at low frequencies, $R_{S i}$ and $C_{S i}$, differs to a large degree from the data. 


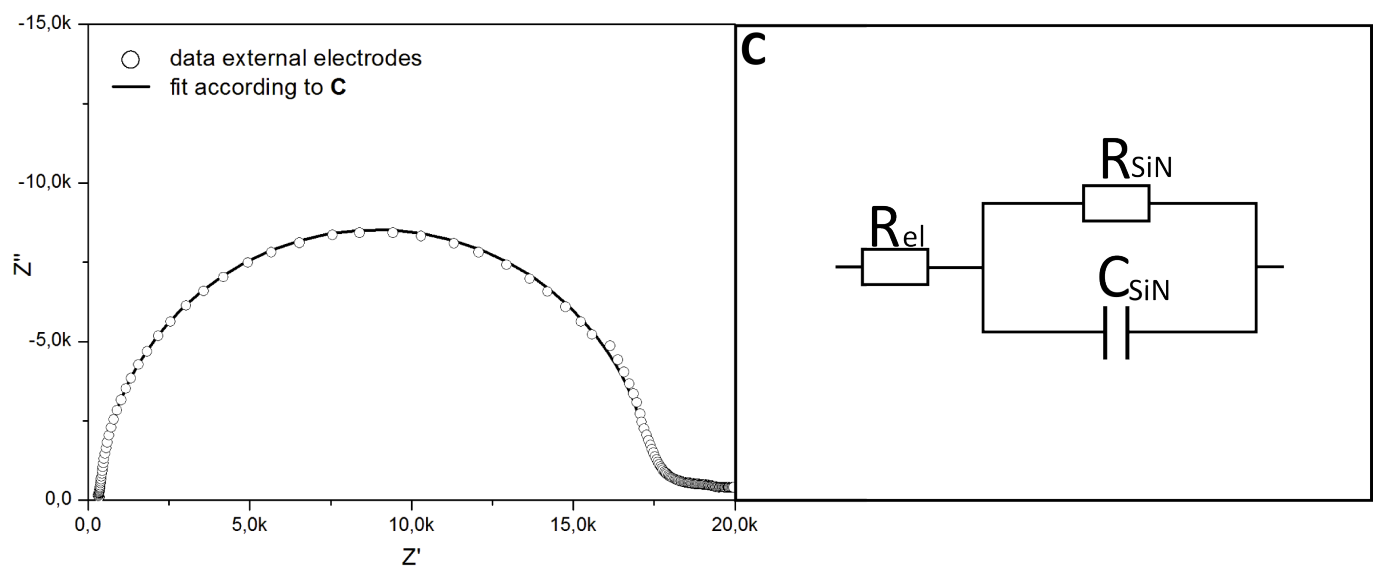

Figure 6.9: Impedance spectra were fitted with an $\mathrm{R}(\mathrm{RC})$ circuit shown in $\mathbf{C}$. Impedance data are displayed as white circles and the fit as a solid line.

The resistance of the empty substrate is defined by the uncovered pores, as theoretical considerations in section 6.2.4 show. The capacitance is dominated by the thin silicon nitride layer.

The parameters that are relevant for the characterization of the substrate in respect to lipid bilayer formation, are therefore the capacitance and the resistance of the pore-bearing silicon nitride layer, which can be found in the high frequency part of the spectra.

Because the dispersion at the low frequency part is very small and not completely shown in the spectra it cannot be fitted accurately with both equivalent circuit models shown in figure 6.7 and figure 6.8. To avoid a wrong interpretation of the data that comes with overcomplicated circuit models, only the part that can be assigned to the pore-bearing silicon nitride membrane was taken into account. The resulting $\mathrm{R}(\mathrm{RC})$ circuit (figure 6.9) was used for all spectra and gives the mean values for the parameters $R_{e l}, R_{S i N}$ and $C_{S i N}$ shown in table 6.1. 
Table 6.1: Parameters of the fitting results for all spectra

\begin{tabular}{|l|l|}
\hline Parameters & Value \\
\hline \hline $\mathrm{R}_{e l}$ & $(1.4 \pm 0.9) \mathrm{k} \Omega$ \\
\hline $\mathrm{R}_{S i N}$ & $(18 \pm 6) \mathrm{k} \Omega$ \\
\hline $\mathrm{C}_{S i N}$ & $(1.3 \pm 0.5) \mathrm{nF}$ \\
\hline \hline
\end{tabular}

The capacitance is of the same order of magnitude as the one obtained by Tiefenauer et al. [42], who used a $300 \mathrm{~nm}$ thick silicon nitride layer as well. The deviations of the spectra can be a result of the different measurement conditions like electrolyte concentration, hydrophobic silanization of the substrate and different pore-sizes and numbers.

\subsubsection{Impedance spectra of the microsubstrates with integrated electrodes}

The impedance spectra change, when integrated electrodes are used instead of the external $\mathrm{Ag} / \mathrm{AgCl}$ wires. In the high frequency part, the same dispersion can be observed as in the spectra recorded with external electrodes, but an additional increase appears at the low frequency part. Spectra that were recorded for the same substrate with external and integrated electrodes only differ in this increase (figure 6.10).
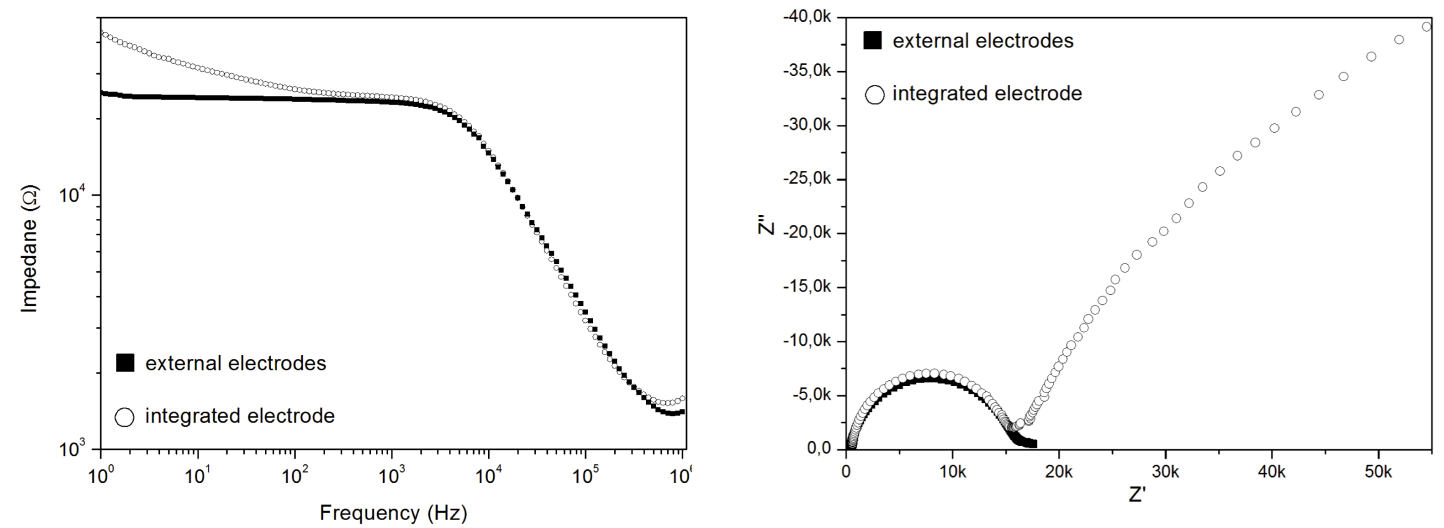

Figure 6.10: The spectra of integrated (white circles) and external electrodes (black squares) differ in the low frequency part. 
The increase in the low frequency part can be modeled with an equivalent circuit containing a resistance in series with a resistor which is in parallel to a CPE (figure 6.11, A.

Despite the strong contribution of the integrated electrodes to the spectrum, all relevant parameters can be extracted. The resistance of the electrolyte solution $R_{e l}$, as well as the resistance and capacitance of the porous silicon nitride layer $R_{S i N}$ and $C_{S i N}$, listed in table 6.2 , coincide within the error margin with those obtained with external electrodes.

Concerning the parameters for the integrated electrodes, $R_{A g / A g C l}$ and $C P E-$ $T_{A g / A g C l}$ show large errors that indicate huge differences between the spectra.

The exponent $C P E-P$ is $(0.6 \pm 0.1)$, which indicates diffusive behavior. Here, the error is not as large as for the other parameters, which means that the diffusive behavior is consistent throughout the experiments. The ideal Warburg impedance with a straight line of $45^{\circ}$, however, cannot be observed.

The differences between the spectra can have several reasons. First of all, only those parameters of the spectrum that can be attributed to the integrated electrodes, $R_{A g / A g C l}$ and $C P E-T_{A g / A g C l}$, show these large errors, whereas for the silicon/silicon nitride substrate, the magnitude of the errors of the parameters $R_{S i N}$ and $C_{S i N}$ is the same as for the values obtained for the external electrodes. This leads to the assumption that only the integrated electrodes differ for different fabrications, whereas the substrate itself is not influenced by the additional fabrication step.

Although the thickness of the deposited silver and the chlorination time are always the same, the thickness of the silver chloride can still vary slightly, which, since the silver layer is so thin, has a large effect on the ratio between silver and silver chloride, and in turn affects the electric properties. Small variations of the chlorination process or the pressure and temperature of the silver deposition process can also alter the porosity of the electrodes. A higher porosity would increase the contribution of diffusive elements to the electric behavior. 


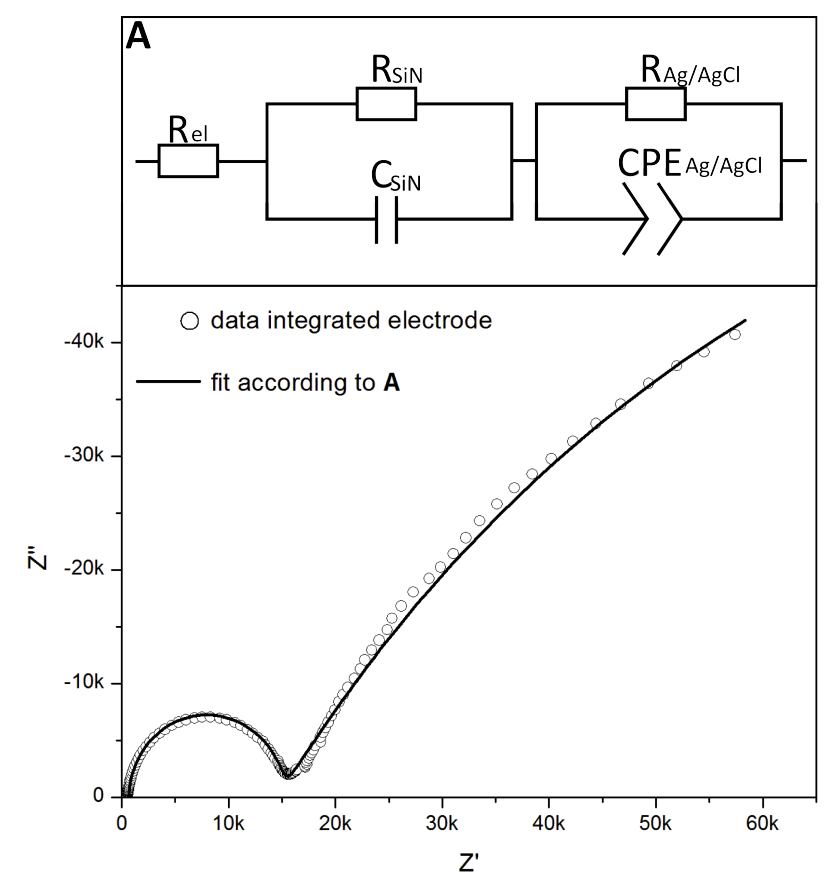

Figure 6.11: The impedance spectra of substrates with integrated electrodes show an additional $\mathrm{R} / \mathrm{CPE}$ element.

This kind of behavior is not uncommon for thin plate $\mathrm{Ag} / \mathrm{AgCl}$ electrodes and was also reported by B. J. Polk et al. [150], who examined the electric behavior of electroplated silver electrodes ( $5 \mu \mathrm{m}$ in thickness) in microchannels using impedance spectroscopy and observed a similar set of spectra. They suggest that the diffusive element comes from the movement of charge across the thin film electrodes, which is probably also the case for the integrated electrodes in the microsubstrate.

Table 6.2: Parameters of the fitting results for all spectra

\begin{tabular}{|l|l|}
\hline Parameters & Value \\
\hline \hline $\mathrm{R}_{e l}$ & $(981 \pm 55) \Omega$ \\
\hline $\mathrm{R}_{S i N}$ & $(18 \pm 8) \mathrm{k} \Omega$ \\
\hline $\mathrm{C}_{S i N}$ & $(1.0 \pm 0.2) \mathrm{nF}$ \\
\hline \hline $\mathrm{R}_{A g / A g C l}$ & $(1.5 \pm 1.8) \mathrm{M} \Omega$ \\
\hline${\mathrm{CPE}-\mathrm{T}_{A g / A g C l}}$ & $(5.8 \pm 7.6) \mu \mathrm{Ss}^{P}$ \\
\hline${\mathrm{CPE}-\mathrm{P}_{A g / A g C l}}$ & $(0.6 \pm 0.1)$ \\
\hline
\end{tabular}


The reason why the integrated electrodes contribute to the impedance spectra at all, whereas the external electrodes are invisible in this frequency range, is probably the significantly larger area of the external electrodes that results in a higher capacitance and therefore shifts this part of the spectrum more to the low frequency part below $1 \mathrm{~Hz}$.

The resistance of the integrated electrodes in the range of several $\mathrm{M} \Omega$ is still small compared to the $\mathrm{G} \Omega$ resistance of the lipid bilayer and does not alter the impedance spectra of the lipid bilayer covered pores. In fact, as shown in the next chapter, no difference between spectra recorded with integrated and external electrodes can be observed.

\subsubsection{Impedance spectra of lipid bilayers on the microsubstrates}

Lipid bilayers were prepared from GUV-spreading on the silicon nitride layer as described in chapter 5.3. The impedance spectra show a single dispersion with an ohmic resistance in the low frequency part. No difference can be detected between the impedance spectra of the integrated and external electrodes in the same experiment, although it was shown that the spectra for external and integrated electrodes differ. The high resistance of the lipid bilayer dominates the spectrum completely and can therefore be extracted very well.

The theoretical lipid bilayer capacitance can be calculated using a capacity of $1 \mu \mathrm{F} / \mathrm{cm}^{2}$ which is the value that was calculated by M. Montal et al. [151] and is commonly used for membrane capacity calculations. The area of the lipid bilayer spanning the 9 pores is $7.1 \mu \mathrm{m}^{2}$ and the capacitance therefore $7.1 \mathrm{fF}$. This is negligibly small compared to the high capacitance of the porous silicon nitride layer with $(1.3 \pm 0.5) \mathrm{nF}$, which adds to the lipid bilayer capacitance. To extract the lipid bilayer capacitance, either the capacitance of the silicon nitride layer has to be decreased by increasing the thickness of the dielectric layer, or the capacitance of the lipid bilayer has to be increased by increasing the area according to equation 6.8. Even thick dielectric coatings result only in a decrease of the capacitance to the $\mathrm{pF}$ range (e.g. $40 \mu \mathrm{m}$ thick layer of SU-8 has decreased the capacitance from $1 \mathrm{nF}$ to $80 \mathrm{pF}$ [117]) which is still high compared to the lipid bilayer capacitance in the $\mathrm{fF}$ range. Increasing the bilayer area has been used as a solution for extracting 
the bilayer capacitance from impedance spectra by Tiefenauer et al. [42]. In this work, however, the preparation method requires very small pore arrays so that no way of extracting the lipid bilayer capacitance could be determined.

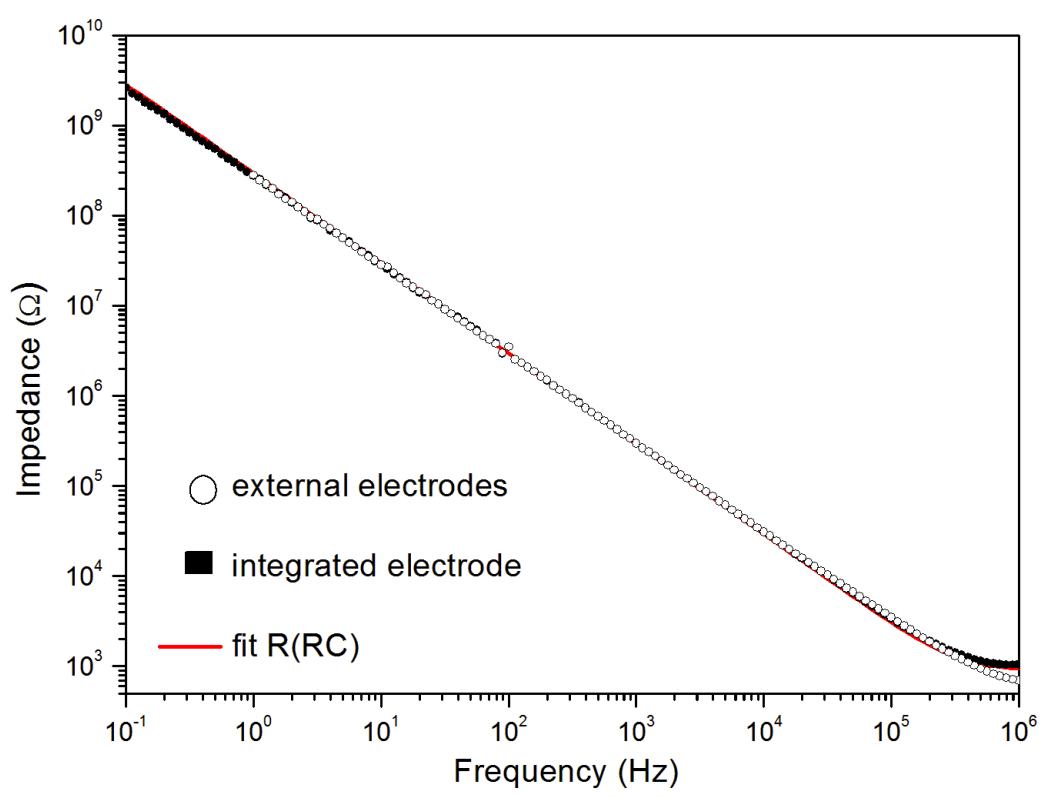

Figure 6.12: Impedance spectra of lipid bilayers recorded with external (white circles) and integrated electrodes (black squares) from the same experiment cannot be distinguished. Both were fitted with an $\mathrm{R}(\mathrm{RC})$ circuit (red solid line).

For fitting the impedance spectra with lipid bilayers a simple $\mathrm{R}(\mathrm{RC})$ circuit was used. Considering the fitting of the empty substrate and the general equivalent circuit for lipid bilayers, which is an RC element, the straightforward approach would be to add the $\mathrm{RC}$ element for the lipid bilayer in series to the $\mathrm{R}(\mathrm{RC})$ circuit that was used for fitting the empty substrate. However, as already pointed out, the capacitance that adds to the one of the empty substrate cannot be resolved in the spectrum and the pore-resistance is dominated by the G $\Omega$ resistance of the lipid bilayer so that the circuit can be simplified to the $\mathrm{R}(\mathrm{RC})$. This procedure is common in literature. Naumann et al. used the same equivalent circuit for fitting the spectra both with and without lipid bilayer for the same reason. They replaced the porous silicon nitride resistance by the membrane resistance and did the same 
with the capacitance. Tiefenauer et al. even reduced the $\mathrm{R}(\mathrm{RC})(\mathrm{RC})$ circuit they used for fitting the empty substrate to a simple $\mathrm{R}(\mathrm{RC})$ circuit, because also here the single circuit elements of the uncovered pores could not be resolved anymore.

\subsubsection{Theoretical pore resistance}

The impedance spectra of the empty substrates show an ohmic resistance that can be assigned to the porous area of the silicon nitride layer. Because this ohmic resistance is the result of the parameters of the pores (i.e. length, shape, radius), a comparison with theoretically calculated values is a good test for the quality of the pore etching. In chapter 4 it has already been shown that the pore shape is round and cylindrical, but all the methods used in this chapter (AFM, SEM and brightfield microscopy) can only provide information on the surface of the substrate or the entry region of the pores, whereas the electric resistance observed in the impedance spectra is a result of the overall pore-shape. This means, if the shape inside the pore or at the exit is completely different from that at the entry (for example different radii throughout the pore) it would have an effect on the resistance.

The theoretical value for the pore resistance is the sum of the resistance inside the pore $\left(R_{\text {pore }}\right)$, at the entry $R_{\text {entry }}$ and at the exit $R_{\text {exit }}$.

$$
R_{\text {total }}=R_{\text {pore }}+R_{\text {entry }}+R_{\text {exit }}
$$

The resistance of the pore is given by the ratio of the length of the pore $l$ and its area $\pi \cdot r_{\text {pore }}^{2}$. The pore resistance therefore depends on the length $l$ of the pore, the radii of both entry and exit resistance $r_{\text {entry }}$ and $r_{\text {exit }}$ and also the resistivity of the electrolyte solution $\kappa$ the experiments were performed in.

$$
R_{\text {pore }}=\frac{l}{\pi \cdot r_{\text {pore }}^{2} \cdot \kappa}
$$

The resistance of the entrance and the exit of the pore can be calculated assuming disk-shaped openings [152, 153]:

$$
R_{\text {entry }}=\frac{1}{4 \cdot r_{\text {exit }} \cdot \kappa} \text { and } R_{\text {exit }}=\frac{1}{4 \cdot r_{\text {exit }} \cdot \kappa}
$$


For the radius, the value for the feret-diameter, determined from the brightfield microscopy images $((1.18 \pm 0.14) \mu \mathrm{m})$ in chapter 4 , was taken, the length of the pore equals the thickness of the silicon nitride layer of $300 \mathrm{~nm}$. With these values the theoretical pore resistance can be calculated to $12.3 \mathrm{k} \Omega$, which is within the error margin for the experimental values for both integrated and external electrodes. The small deviation of the experimental from the theoretical values can have several reasons. First, the pores are not completely cylindrical at the exit and entry as it can be seen in the SEM images (figure 4.8), but the edges are rather smooth resulting in a rounded entry region to the pores which are not as sharp as they would be for completely cylindrical pores as figure 6.13 shows. Because of that also the radius, which was obtained by adjusting a threshold to the entrance of the pores, could be overestimated so that the radius used for the calculation of the pore resistance was too large, which in turn would decrease the theoretical resistance. Another source for the deviation is the electrolyte conductivity $\kappa$. Here, the literature value was taken for calculating the resistances, but $\kappa$ is very dependent on the temperature and can also change as the local concentration changes during the experiment. But as the experimentally determined pore resistance is within the error margin to the theoretically calculated ones, these effects do not seem to be large.

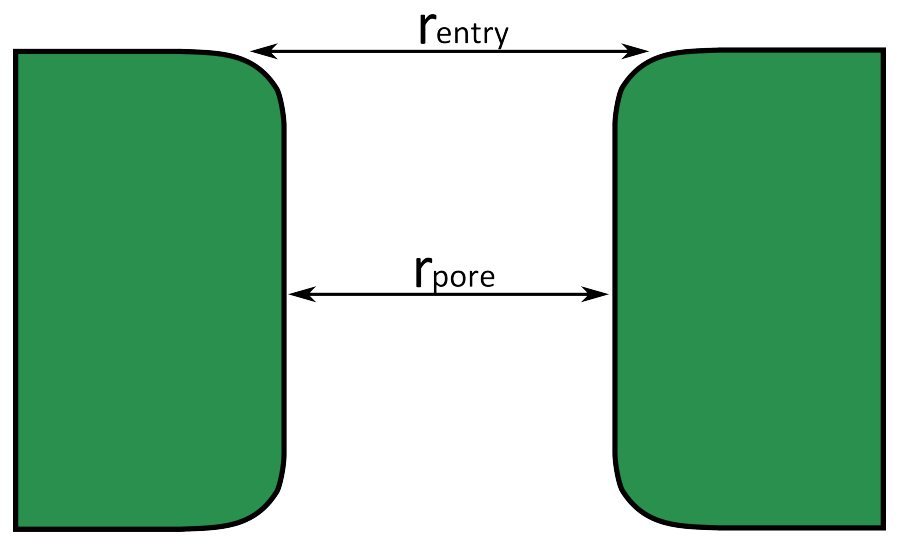

Figure 6.13: The round edges of the pores lead to a an entrance radius $r_{\text {entry }}$ that is higher than the actual pore radius $r_{p o r e}$.

However, around $30 \%$ of the substrates showed a considerably higher resistance of $(81 \pm 9) \mathrm{k} \Omega$ in the experiments. This means that some pores are not etched 
completely during the substrate fabrication. So although all substrate are inspected before use, impedance spectroscopy seems crucial for verifying the success of the substrate fabrication. In single channel experiments the difference between 15 $\mathrm{k} \Omega$ and $81 \mathrm{k} \Omega$ cannot be distinguished, because such high currents are beyond the bandwidth of the amplifier used for these experiments. For the analysis of the electrical properties of the substrate shown in this chapter, as well as for the single channel experiments, only those $60 \%$ of the substrates with sufficiently low resistances were used.

\subsubsection{Capacitance from triangle voltage pulses}

The single channel amplifier (ELC03XS) cannot be used for the investigation of the electrical properties of the empty substrate. However, as soon as a lipid bilayer covers the pores and the current is sufficiently low, the total resistance as well as the total capacitance of the substrate can be determined. This is important, because switching between the impedance spectroscopy setup and the single channel recording setup may disturb the experiment, even though only the electrodes have to be reconnected (chapter 5.2). Also after some measuring time it is reasonable to control the membrane and make sure that no clogging of the pores has occurred. The ohmic resistance is calculated automatically by the measurement software (Patchmaster, HEKA Elektronik, Lambach, GE) by dividing the holding potential by the detected current response. This provides a permanent control of the membrane resistance between the measurements.

Additionally, the capacitance can be controlled by applying triangle voltage pulses. According to equation 6.18

$$
I_{g e s}=I_{\Omega}+I_{C}=I_{\Omega}+C \frac{d V}{d t}
$$

the capacitance $C$ can be determined from the total current $I_{\text {ges }}$, if the ohmic resistance is large enough that the corresponding current $I_{\Omega}$ can be neglected, and if $\frac{d V}{d t}=1 \mathrm{~V} / \mathrm{s}$ (figure 6.14). This can be achieved by applying a triangular voltage pulse with the slope of $1 \mathrm{~V} / \mathrm{s}$. At a sufficiently low current $I_{\Omega}$ the response is a square current and the capacitance is the difference between the two levels (figure $6.15)$. 


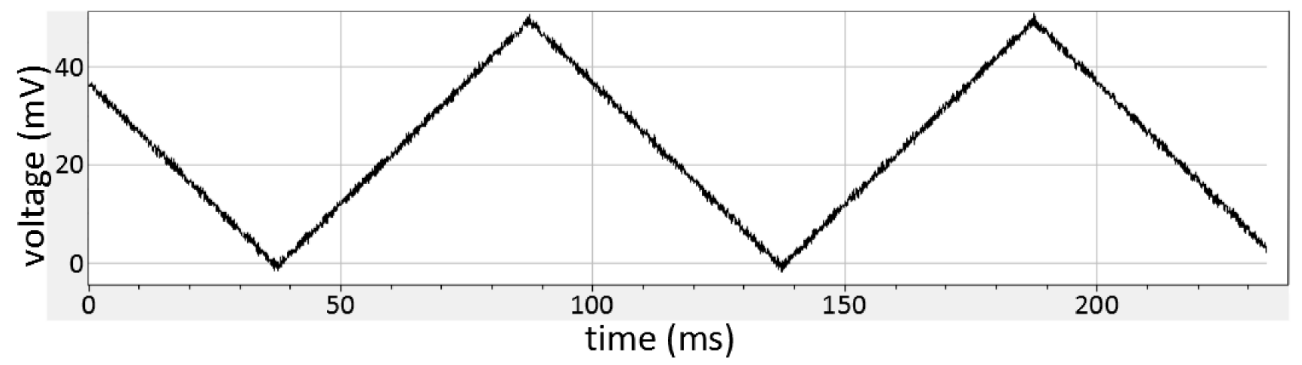

Figure 6.14: The capacitance can be calculated from the current response of a triangular voltage pulse with a slope of $1 \mathrm{~V} / \mathrm{s}$.

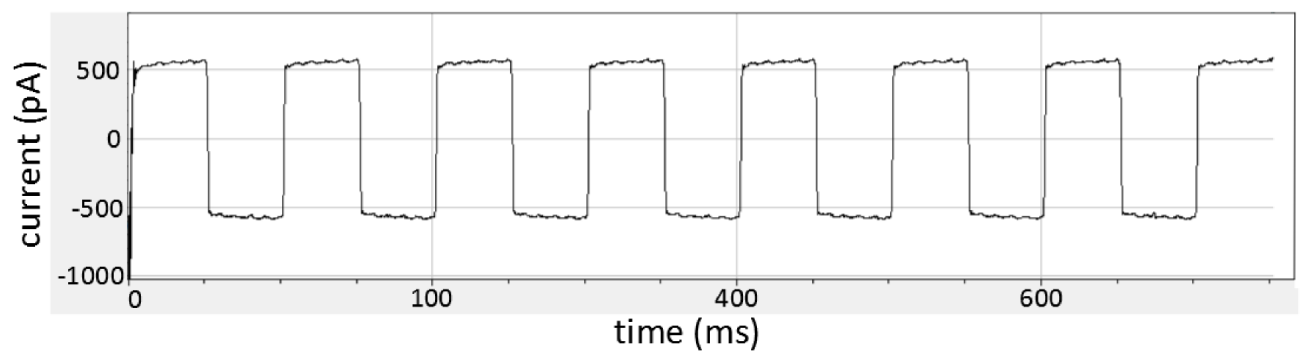

Figure 6.15: The current response to a triangular voltage pulse at a high ohmic resistance.

For obtaining the capacitance, a triangular voltage with an amplitude of $50 \mathrm{mV}$ and a duration of $50 \mathrm{~ms}$ was applied and the current response was recorded. The resulting capacitances were between 0.8 and $1.1 \mathrm{nF}$ for both integrated and external electrodes which is very close to the values obtained by impedance spectroscopy. Although impedance spectroscopy is a much more accurate method for resolving the contribution of different components to the overall resistance and capacitance, the method described here is a quick and easy way of checking the integrity of the lipid bilayer during the measurement.

\subsubsection{Experimental procedure}

Before lipid bilayer preparation, an impedance spectrum of the empty substrate was taken to confirm proper etching of the pores and sealing of the sample chamber. For this reason, the sample chamber and the substrate were assembled as described 
in section 5.2. The lower electrolyte compartments were filled with buffer $(1 \mathrm{M} \mathrm{KCl}$, $10 \mathrm{mM}$ MES, $10 \mathrm{mM}$ MES, $0.06 \mathrm{mM}$ EDTA, pH 7.0) so that no bubbles were left in the channels and $300 \mu \mathrm{l}$ of the same buffer was pipetted into the top electrolyte compartment. An $\mathrm{Ag} / \mathrm{AgCl}$ wire was used as an electrode on the trans side and a silver wire for the connection to the integrated electrode on the cis side.

The spectra were taken with a VersaStat 4 impedance spectrometer (Princton Applied Research, Oak Ridge, US) at $10 \mathrm{mV}$ voltage amplitude to ensure linearity in a two-electrode configuration. The spectrometer was grounded to the faraday cage with an additional cable.

Before lipid bilayer formation, the buffer in the top compartment was almost completely removed, to avoid dilution of the GUV solution. Lipid bilayers were prepared as described in section 5.3. 


\section{CHAPTER 7}

\section{Alamethicin single-channel experiments}

\subsection{Voltage clamp single-channel recordings}

In contrast to impedance spectroscopy that records the frequency dependent resistance (chapter 6), voltage clamp techniques use DC voltages that are either held at a single potential or follow a controlled pattern of voltage pulses to obtain information on ion channels in the cell membrane or in artificial lipid bilayers. Voltage clamp techniques were developed in the 1940s and 50s by K. S. Cole and A. L. Hodgkin for the electrical examination of the squid giant axon [154, 155]. Since then, the range of applications for this method has expanded from wholecell recordings, where collective properties of ion channels overexpressed in a cell are tested and, on the other side, single-ion channels are recorded in patch-clamp experiments, where a small patch of membrane is removed from a cell with a micropipette electrode. A similar approach is single channel bilayer recording, where an artificial membrane separates two electrolyte compartments. This technique is also used in this work. Because the conductances of single ion channels are very small (in the range of $\mathrm{pS}$ to $\mathrm{nS}$ ), voltage clamp systems are constructed for high amplification of small signals, which means that the bandwidth of the amplification system is restricted to signals in the $\mathrm{nA}$ and $\mathrm{mV}$ range respectively. This is important because it indicates that the ohmic resistance and especially the capacitance of the empty micro-substrate cannot be obtained as its conductance is larger than the bandwidth of the recording system.

In single ion channel voltage clamp recordings with planar lipid bilayers (BLMs), the membrane capacitance is usually used to verify lipid bilayer formation. This 
is possible because in BLM systems, the lipid bilayer capacitance is the dominating part in the total capacitance. If, for example, a lipid bilayer is prepared in a $100 \mu \mathrm{m}$ aperture in a teflon sheet with a thickness of $100 \mu \mathrm{m}$, the capacitance of the perforated teflon sheet is much smaller compared to the $300 \mathrm{~nm}$ thick silicon nitride membrane (according to equation 6.8). Also, the lipid bilayers are prepared in much larger apertures which increases their capacitance by increasing their area. For the microporous substrates, this verification of membrane formation is therefore not possible, because, as the impedance spectroscopy experiments showed, the capacitance of the lipid bilayers is too small to be distinguished from the substrate's capacitance and only the parameters of the system after lipid bilayer formation are accessible. A high ohmic resistance alone is, however, not enough to be sure the substrate is covered by a lipid bilayer, because this can also be caused by air bubbles trapped in the pores or debris clogging the pore. For this reason, impedance spectroscopy was implemented. Here, the comparison of the empty and the lipid bilayer covered substrate can be used to monitor lipid bilayer formation. On the other hand, impedance spectroscopy cannot resolve single ion channels incorporated in a lipid bilayer.

The combination of impedance spectroscopy and single channel voltage clamp provides information on the electrical properties of the whole system as well as single channel resolution. In this chapter, the results for single channel recordings of the antimicrobial peptide alamethicin are presented.

\subsubsection{Single-channel recording and analysis}

Single channel events were recorded with an ELC03XS amplifier (NPI Electronics, Tamm, GE) and the Patchmaster software (HEKA Elektronik, Lambrecht, GE) was used for data display. The holding potential was set to a specific value via the software and the current response was recorded at a sampling rate of $50 \mathrm{kHz}$. The data were filtered first at $1 \mathrm{kHz}$ by a lowpass 4-pole Bessel filter included in the amplifier and again digitally using a $100 \mathrm{~Hz}$ digital filter for enhancing the signal to noise ratio. Since all digital filters are reversible, the frequency cutoff for the hardware filter was set as high as possible. The current output amplification was set to $2 \mathrm{~V} / \mathrm{nA}$ for Alamethicin single channel recordings.

For data analysis, the Clampfit software (Axon Instruments, Union City, US) was 
used. All data traces were filtered digitally with an 8-pole Bessel type lowpass filter at a $100 \mathrm{~Hz}$ cutoff frequency to enhance the signal to noise ratio. With the " single channel search" option the gating of single or multiple ion channels can be evaluated. A marker is set manually for each level that corresponds to the opening of one channel. The example in figure 7.1 shows four different levels (blue lines) with level 0 as the baseline, where all channels are closed. The software recognizes each change between the levels and edits the current difference in pA. Additionally, in case of single channels, where only two levels are distinguished, the duration of the event is edited. This is defined as the transition from level 0 to level 1 and back.

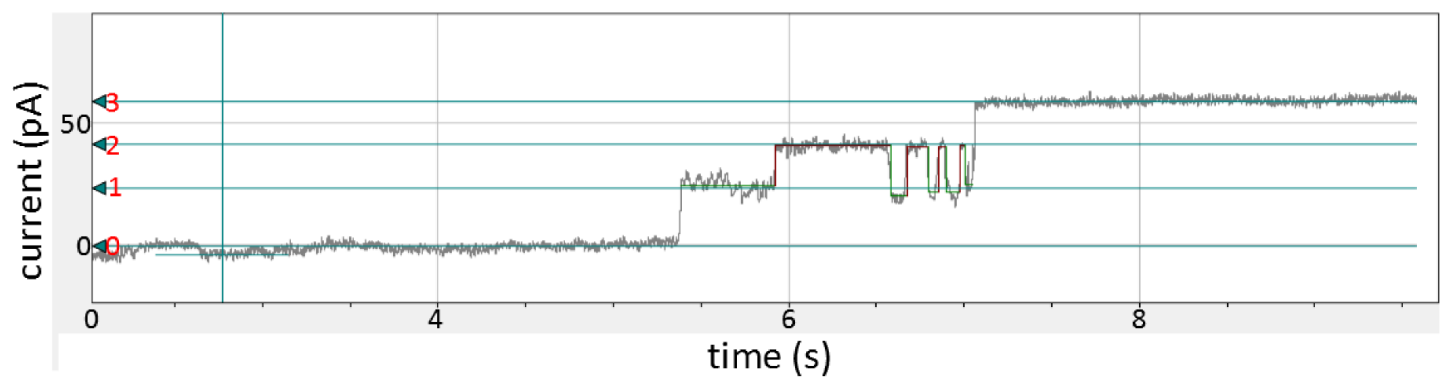

Figure 7.1: Example single-channel recording with four conductance levels (blue lines 0-3) set with the data anaysis software Clampfit.

\subsection{Alamethicin}

Alamethicin is a channel forming antimicrobial peptide consisting of 20 amino acids that are arranged in an $\alpha$-helix. It is an antibiotic that can be found in the fungus Trichoderma veride and self-assembles into lipid bilayers forming water-filled pores. The number of alamethicin monomers making up one pore changes and is responsible for the individual conductance states. During an experiment, the monomers diffuse to and away from the pore so that the number of monomers is transient and multiple conductance states can be observed [156].

Data sets for the conductance levels for alamethicin have been recorded by many groups for different conditions. The conductances and the preference for the conductance levels depend on the applied voltage, the concentration of the electrolyte 
solution and on the intrinsic tension of the lipid bilayer. The ion channel is only weakly selective towards cations [156].

For $1 \mathrm{M} \mathrm{KCl}$ as an electrolyte solution, Sansom et al found conductance states of 19, 280, 1300, and $2700 \mathrm{pS}$ corresponding to 4-7 monomers [156]. Tielemann et al simulated different conductance states for 4-8 monomers per pore [157] and found values of $20,180,250,310,370 \mathrm{pS}$.

As it can be reconstituted easily and is commercially available, alamethicin is one of the best studied ion channels in artificial membrane systems and is also often used for proof of concept experiments.

\subsubsection{Results and discussion of alamethicin single-channel experiments}

The single channel traces obtained from alamethicin show different conductances (figure 7.2), as described above. The opening events were analyzed as shown in section 7.1.1. The conductances of all events recorded at a holding potential of $100 \mathrm{mV}$ were calculated and plotted in a histogram which was fitted with gaussians. The histogram shows three conductance levels at $(176 \pm 7) \mathrm{pS},(486 \pm 8) \mathrm{pS}$ and $(837 \pm 11) \mathrm{pS}$ (figure 7.3$)$ with the majority of events at the lowest conductance state. Also, few events at conductances of $(1448 \pm 77) \mathrm{pS}(\mathrm{n}=6)$ and $(3697 \pm 206) \mathrm{pS}$ $(\mathrm{n}=5)$ were found. Figure 7.2 shows representative channel recordings that show examples for all conductance states. The conductance levels found here correspond to those described above, except for the lowest level around $20 \mathrm{pS}$ which was not observed. The variations between the conductance levels can be a result of the different voltages used. 

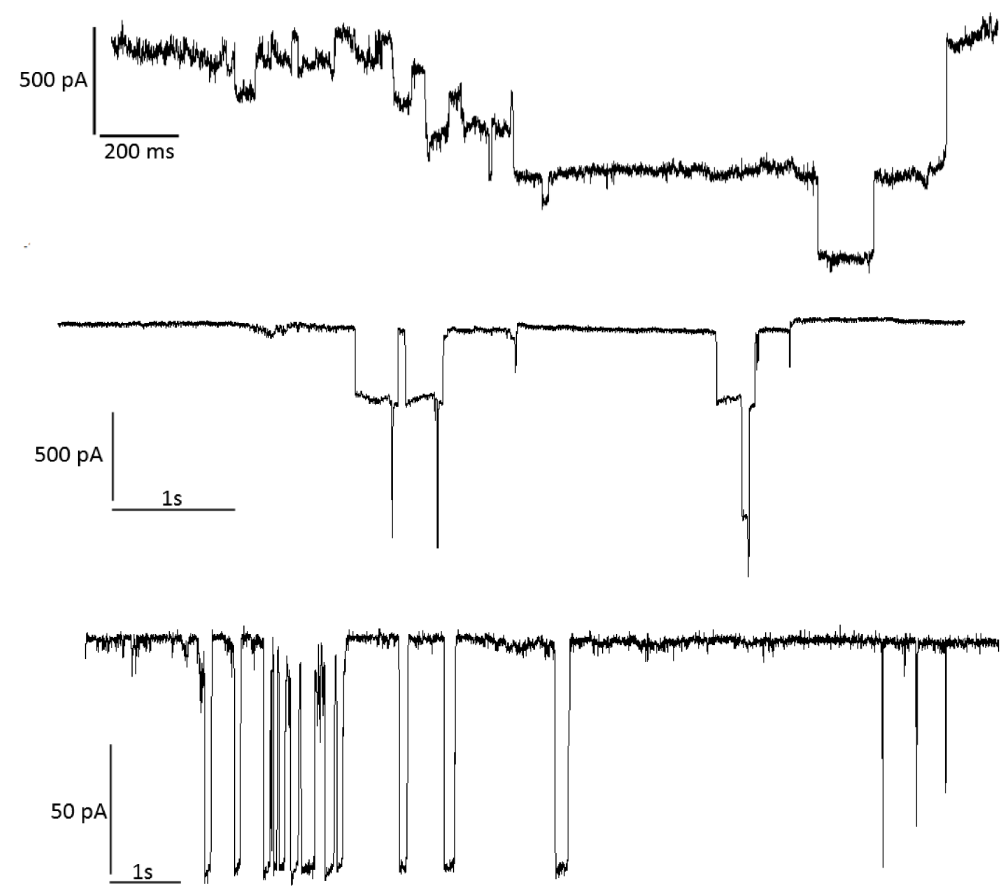

Figure 7.2: Representative single channel traces obtained for alamethicin in DOPC lipid bilayers in $1 \mathrm{M} \mathrm{KCl}$ solution.

Alamethicin is a stretch activated ion channel and therefore sensitive towards prestress in the lipid bilayer. The probability of occupying a subsequently higher conductance level increases exponentially with membrane tension [67], but the membrane tension does not alter the channel conductances. This has been shown by L. R. Opsahl and W.W. Webb, who applied stress to the membrane using a patch pipette [67], and by E. Stava et al., who observed the same behavior for alamethicin in mechanically stretched planar membranes [56].

Lipid bilayers on unfunctionalized porous substrates have a lower surface tension than those that are tethered to the solid parts, for example by thiol-gold functionalization, as it has been shown for example by I. May et al [41]. This means that the reason for the observation of only few high conductances states in this work is probably the low surface tension of the lipid bilayer. Additionally, S. L. Keller et al [158] showed that lower conductance levels are also preferred in DOPC lipid bilayers compared to DOPE, an effect that they attribute to the lower curvature of DOPC. As lipid bilayers in this work were also prepared from pure DOPC, the 
same effect may cause the preference for the low conductance states here.

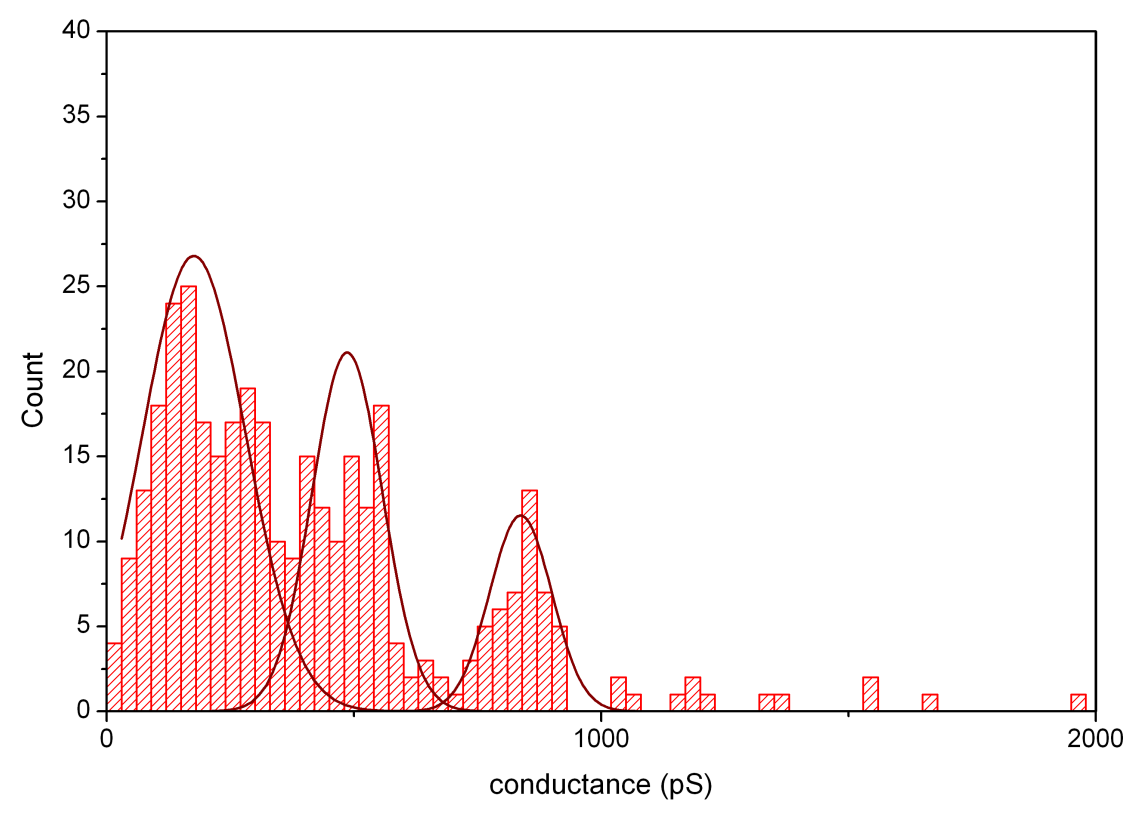

Figure 7.3: The conductance histogram from all single channel events recorded at $100 \mathrm{mV}$ show three main conductance levels at $(176 \pm 7) \mathrm{pS},(486 \pm 8) \mathrm{pS}$ and $(837 \pm 11) \mathrm{pS}$ and few higher conductance levels at $(1448 \pm 77) \mathrm{pS}(\mathrm{n}=6)$ and $(3697 \pm 206) \mathrm{pS}(\mathrm{n}=5)$.

These proof of concept experiments show that alamethicin ion channels can be reconstituted into lipid bilayers on the microporous substrate and that several conductance levels can be observed. Alamethicin is especially interesting for future experiments, as it is mechanosensitive. In low tension lipid bilayers, like in this work, the low conductance states are preferred and the substrate offers various possibilities for modification of membrane tension such as hydrostatic pressure, functionalization or stress application by an optical trap so that inducing higher conductance levels could be applied as a probe for changes in membrane tension. 


\section{CHAPTER 8}

\section{Polycystin-2}

Polycystin-2 (TRPP2, PC2), the gene product of PKD2 [159], is a member of the TRP (transient receptor potential) family [63]. In a mutated form, it is one of the two integral membrane proteins that cause autosomal dominant polycystic kidney disease (ADPKD), the other one being polycystin-1 [159]. Thus, it is also of medical interest. This work is embedded in a project that aims to investigate polycycstin-2 with respect to its mechanosensitive properties in in vivo and in vitro experiments. The in vitro reconstitution of the protein into free-standing lipid bilayers requires an expression strategy, which was adopted from the work done by the group of $\mathrm{H}$. Cantiello [160, 161] and modified to be established for future experiments.

In this chapter, a review on the polycystin-2 properties will be given and the cellfree expression system used will be explained. 


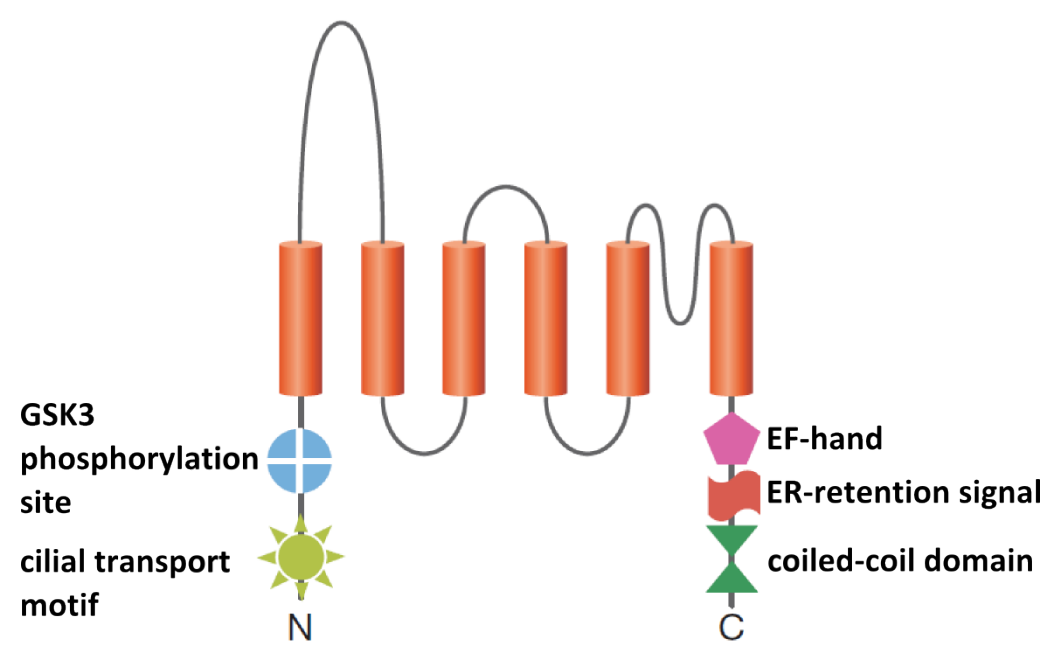

Figure 8.1: Schematic structure of polycystin-2, adapted from [162]

Polycystin-2 is an integral membrane protein with a molecular weight of 110 kDa (968 aa) [159] and possesses six transmembrane helices with cytosolic C- and N-termini. The C-terminal end of polycystin-2 contains an EF-hand for calcium binding (figure 8.1), a coiled-coil-domain that is crucial for association with other proteins such as polycystin-1 [160] and an ER-retention signal [163]. The N-termius contains a phosphorylation site and a cilial transport motif [162].

Polycystin-2 associates with a variety of other proteins that alter its function considerably. About half of the known binding partners are cytoskeleton-associated $(\alpha$-actinin, kinesin2-motor subunits KIF3A and KIF3B, Hax-1, Tropomyosin-1, Troponin-1), which suggests a direct interaction with the cytoskeleton [70].

Polycystin-2 is located in the endoplasmic reticulum (ER), the plasma membrane and primary cilia [162]. In the ER, the channel acts as an intracellular calcium release channel which was shown in in vitro single channel experiments with ER membranes fused to BLMs [164]. It interacts with and modulates the function of Inositol-triphosphate receptor proteins [165].

In the plasma membrane, polycystin- 2 accociates with the C-terminal region of polycystin-1, a transmembrane glycoprotein, via its C-terminus. The polycystin1 / polycystin-2 complex is thought to work as a receptor ion channel (chapter 2.4), where polycystin- 1 transduces signals from the environment to polycystin- 2 which in turn regulates the $\mathrm{Ca}^{2+}$ flux $[160,166]$. It was also shown that polycystin- 
1 reverses the inhibitory effect polycstin- 2 has on the activity of stretch activated channels in the plasma membrane [64]. In vitro experiments in which $\mathrm{Ca}^{2+}$ was depleted with chelators showed a significant effect on the ion channel conductance of polycystin-2 reconstituted from human placenta membranes, but not on polycystin2 obtained from cell-free expression, indicating that other proteins present influence polycystin-2 function [167]. It co-localizes with other members of the TRP familiy, for example TRPV4 and TRPC1 which form heterotetramers with polcystin-2. Because both TRPV4 and TRPC1 contain ankyrin-repeats in their N-termini, which are important for sensing mechanic stimuli and attachment of membrane proteins to the cytoskeleton, it was proposed that they form flow-sensing complexes with polycystin-2. For TRPV4, this role as a flow sensing complex was shown in renal cilia of epithelial cells [73, 168, 169].

It is also known that the channel open probability of polycystin-2 increases when fibrocyctin and the kinesin-2 motor subunit KIF3B are present [69]. The same can be observed for the association with $\alpha$-actinin [70] which seems to indicate an influence of the cytoskeleton to polycystin-2 function.

Polycystin-2 is involved in the flow sensing in primary cilia of epithelial cells and embryonic nodal primary cilia [70], where it is also involved in establishing leftright symmetry [170]. Apart from TRPC1 and TRPV4, it also forms a complex with polycystin-1, which is involved in flow-induced calcium signalling [171].

Several in vitro studies, mainly conducted in the group of H. Cantiello, investigated the single channel conductance for different preparations [172, 62], the voltage and pH sensitivity [161] and the effect of polycystin-1 [160]. For polycystin-2 purified from human placenta membranes (human syncytiotrophoblasts), expressed in a cell-free expression system and inside-out patches from Sf9 insect cells, the conductance levels were similar around $177 \mathrm{pS}(15 \mathrm{mM} \mathrm{KCl}$ (cis) and $150 \mathrm{mM} \mathrm{KCl}$ (trans)) and $134 \mathrm{pS}(150 \mathrm{mM} \mathrm{KCl} \mathrm{(cis)} \mathrm{and} \mathrm{(trans))} \mathrm{with} \mathrm{several} \mathrm{subconductance}$ states. The open probability was shown to be highly dependent on $\mathrm{pH}$ and holding potential; a low $\mathrm{pH}$ increases the probability of the channel to be in the open state. Reconstituted polycystin-2 from the cell-free expression showed spontaneous and voltage induced inactivation. Activity was regained after adding the C-terminal region of polycystin-1 to wt-polycystin-2, indicating that this channel is directly regulated by binding partners. 


\subsection{Polycystin-2 expression}

\subsubsection{Vector and restriction digest}

For the expression of polycystin-2, an in vitro reticulocyte transcription/translation system (Promega, Madion, US) was used according to Cantiello et al [160, 172, 62], who also kindly provided the vector (pc-DNA 3.1) containing the PKD2 open reading frame (figure 8.2) between Nde1 (aa 484) and the T7 promoter. Replication occurred in DH5 $\alpha$ cells that were transfected by electroporation. The purification was done by Midiprep. 


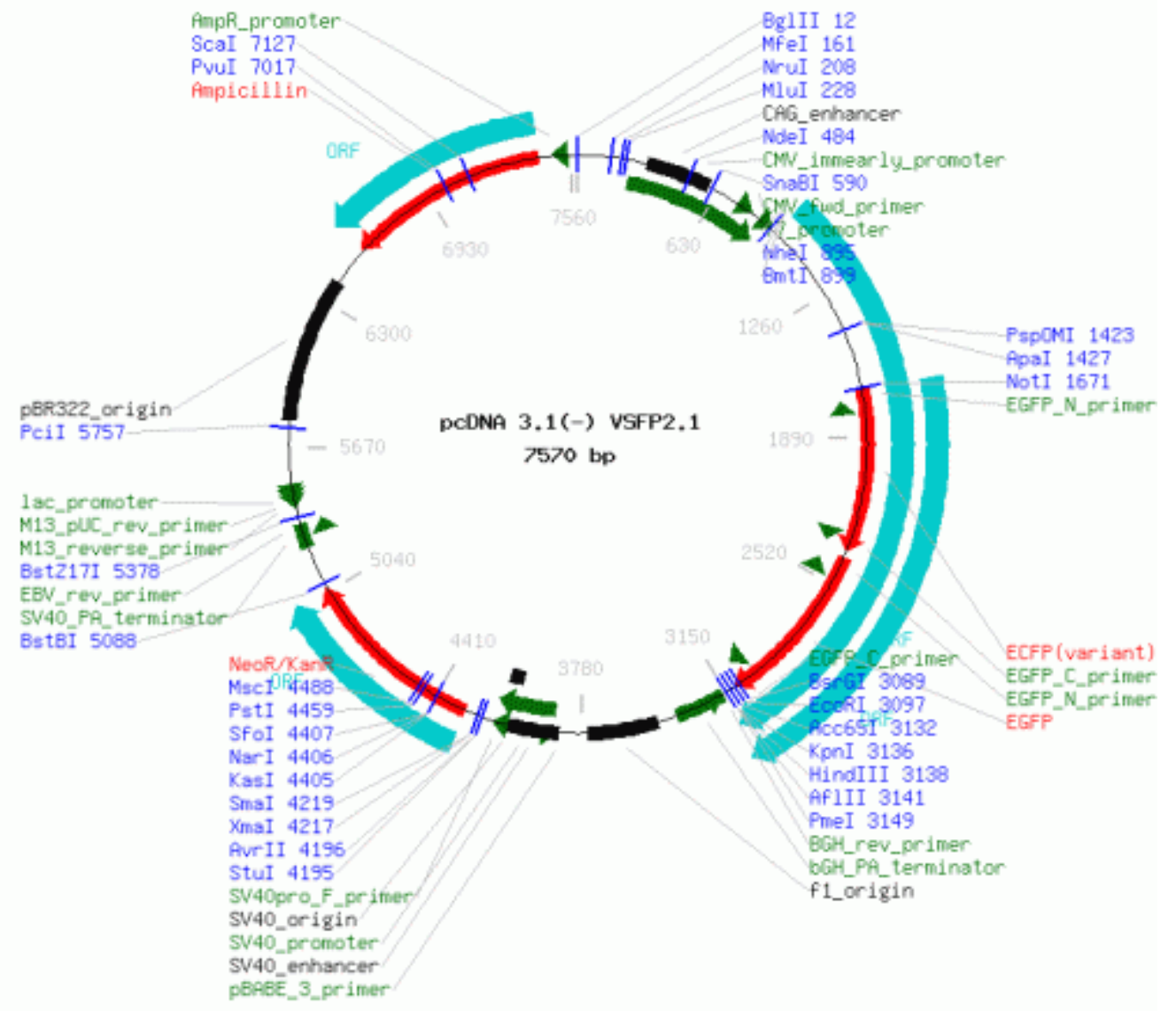

Figure 8.2: The vector pc-DNA3.1/HisC containing polycystin-2 between position 484 and the T7 promoter was used for the cell-free expression.

The vector, including the polycystin- 2 sequence, contains about $10.9 \mathrm{~kb}(5.4 \mathrm{~kb}$ polycystin-2 [173] and $5.5 \mathrm{~kb}$ vector). A restriction digest with Nde and Xho (figure 8.3) shows the undigested vector (column 2, pure) in coiled, supercoiled and linear conformation. The digest with either Nde (column 3) or Xho (column 4) shows the band of the single cut DNA at about $10 \mathrm{~kb}$. This band can also be seen in column 5 , which shows the fragments after double digestion. The other two bands indicate the fragments containing the polycystin- 2 sequence $(\sim 5.9 \mathrm{bp})$ and the rest of the vector $(\sim 4.9 \mathrm{~kb})$. 


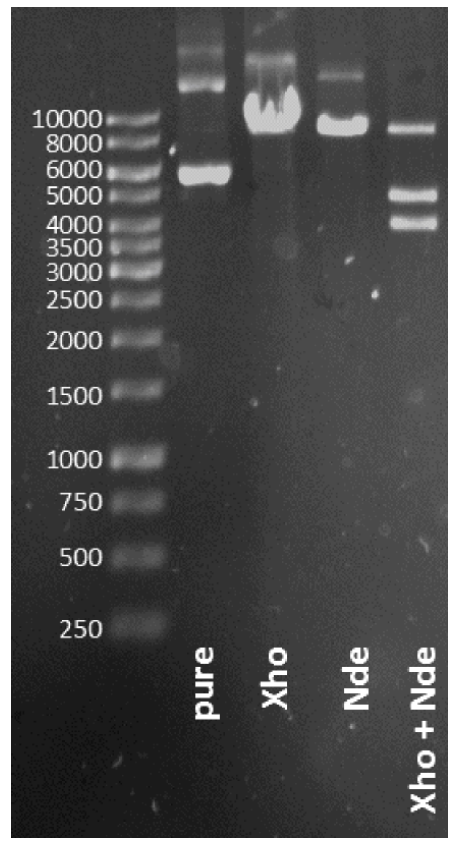

Figure 8.3: Result of the restriction digest of the polycystin- 2 containing vector pc-DNA3.1/HisC before the digest (column 2, pure), after digest with Nde (column 3 , Nde), after digest with Xho (column 4, Xho) and after digest with both Nde and Xho (column 5, Nde + Xho). Column 1 shows a marker for orientation.

\subsubsection{Dot blot}

When a new antibody is used for the detection of a protein, the concentration has to be optimized. This can be done by performing a dot blot, which is similar to a Western blot, with the difference that protein and antibody, or primary and secondary antibody in this case, can be applied directly on nitrocellulose membranes without separating or analyzing the reactants with SDS page first. Here the dot blot was used to determine a suitable concentration of the primary antibody against polycystin-2 (H-280-sc-25749, a rabbit polyclonal antibody raised against amino acids 689-968 of polycystin-2 of human origin, Santa Cruz Biotechnology) and a suitable secondary antibody. Anti-polycystin-2 antibody dilutions tested were 1/200 A, 1/400 B, 1/100 C, 1/1000 D. Also, for control, rabbit HA (E) and rabbit $\mathrm{CM}(\mathbf{F})$ antibodies (positive control) and a mouse antibody (G, negative control) were tested. Secondary antibodies were two anti-rabbit (2005 (I) and 2009 (II) and an anti-mouse antibody (III) for negative control. 

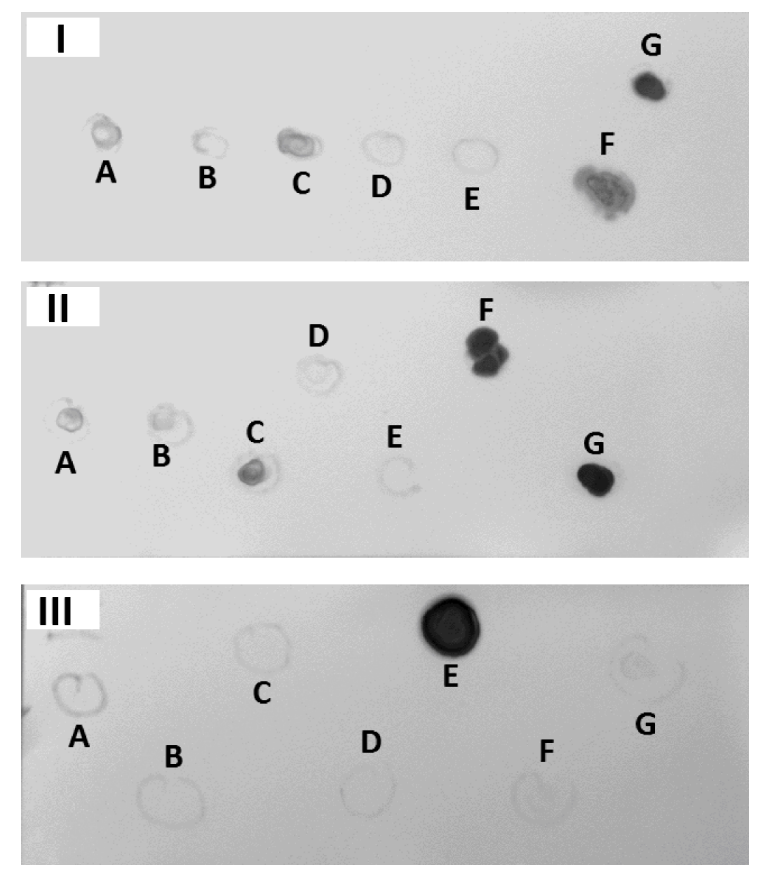

Figure 8.4: Dot blot for the determination of a suitable secondary antibody and dilution of the primary antibody. I-III are secondary antibodies: anti-rabbit antibody 2005, anti-rabbit antibody 2009 and anti-mouse antibody. The dilutions of the primary antibody are (A 1/200, B 1/400, C 1/100, D 1/1000). For positive control two anti-rabbit antibodies $\mathbf{E}$ and $\mathbf{F}$ were used, for negative control anti-mouse antibody $\mathbf{G}$.

The results obtained from the dot blot are shown in figure 8.4. The positive controls $\mathbf{E}$ and $\mathbf{F}$ show coloring with both anti-rabbit antibodies (I and $\mathbf{I I}$ ) but not with the anti-mouse antibody (III), the negative control $(\mathbf{G})$ only shows coloring with the anti-mouse antibody but not with the two anti-rabbit antibodies. The polycystin-2 antibody shows coloring with the highest intensity at a dilution of 1/100 with both anti-mouse antibodies with a slightly higher intensity at II.

In the SDS-page/Western blot analysis used for the analysis of the cell-free expression of polycystin-2, the anti-polycystin- 2 antibody was used in a dilution of $1 / 100$ with the secondary anti-rabbit antibody 2009 (III). 


\subsubsection{Cell-free expression of polycystin-2}

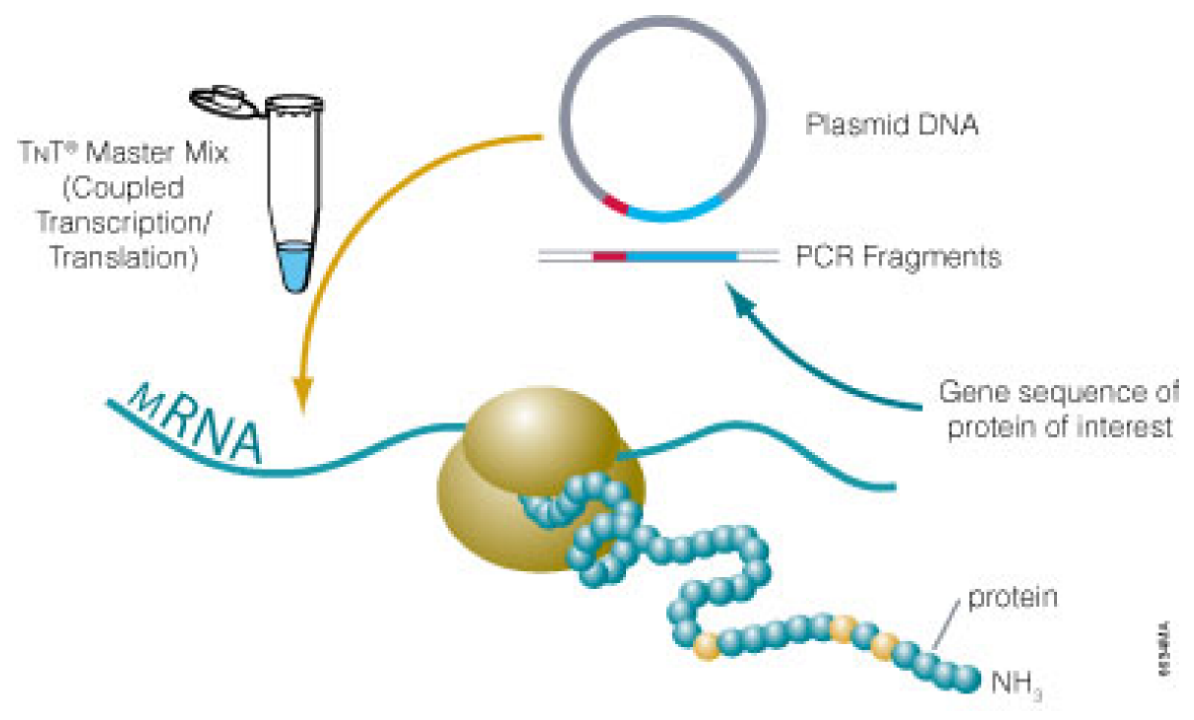

Figure 8.5: Scheme for the cell-free expression of polycystin-2 [174].

For the transcription/translation reaction, the cell lysate (Master Mix) containing the transcription and translation machinery of the cell and the amino acids except methionine (which was added before the reaction either radioactively labeled or unlabeled) and the plasmid were mixed and brought to a total volume of $50 \mu \mathrm{l}$ by the addition of nuclease-free $\mathrm{H}_{2} \mathrm{O}$ as shown in table 8.1. The reaction mix was heated to $30^{\circ} \mathrm{C}$ for $90 \mathrm{~min}$ and then cooled in ice to stop the reaction.

Table 8.1: Components and volumes for the cell-free expression of polycystin-2.

\begin{tabular}{|l|l|}
\hline Component & Volume \\
\hline \hline Master Mix & $40 \mu \mathrm{l}$ \\
\hline Met 1 mM & $1 \mu \mathrm{l}$ \\
\hline DNA & $1-3 \mu \mathrm{l}$ \\
\hline $\mathrm{H}_{2} \mathrm{O}$ & $8-6 \mu \mathrm{l}$ \\
\hline Total & $50 \mu \mathrm{l}$ \\
\hline
\end{tabular}

The analysis of protein expression in a cell-free expression system is usually done by either incorporating fluorescently labeled amino acids or by audioradiography. 
For audioradiography, radioactively labeled amino acids, mostly methionine or cysteine containing $\left[{ }^{35} \mathrm{~S}\right]$, but also $\left[{ }^{3} \mathrm{H}\right]$ leucine or $\left[{ }^{14} \mathrm{C}\right]$ leucine, are added to the reaction mix and after SDS page the expression level can be analysed with a Scintillation Counter. As the equipment for audioradiography was not available and the labeling of amino acids can affect the protein functionality, the analysis was done by Western blot instead.

Since the protein cannot be purified due to the low expression level (in the literature, the whole reaction mix was used for the proteoliposome preparation without purification), the primary antibody has to be very specific. SDS page gels show many bands which contain the whole reaction mix and are the same for the negative control and the reaction mix containing DNA. No single band at $110 \mathrm{kDa}$ can be distinguished (figure 8.6).

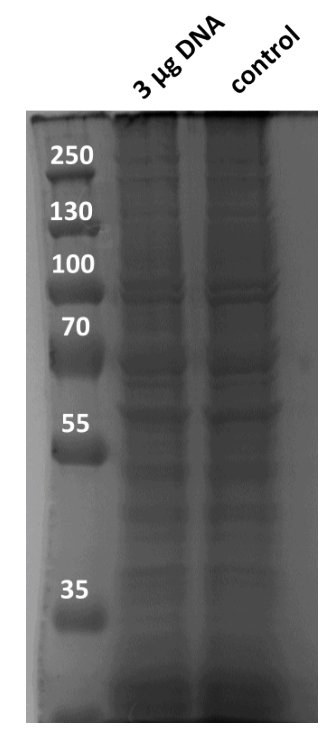

Figure 8.6: SDS-page obtained from the whole reaction mix of the cell-free expression. Column one shows the result of the reaction with $3 \mu \mathrm{g}$ DNA and column two the negative control without DNA.

First attempts of using the His6-tag and a suitable anti-His6-antibody turned out to be too unspecific. The antibody simply has too many binding opportunities in such a crowded sample so that no results were obtained in the Western blot. Therefore, an antibody raised against the last 279 C-terminal amino acids of PC2 
itself was used. This was specific enough to detect the protein in the Western blot. A suitable concentration of the primary and secondary antibody was determined by dot blot.

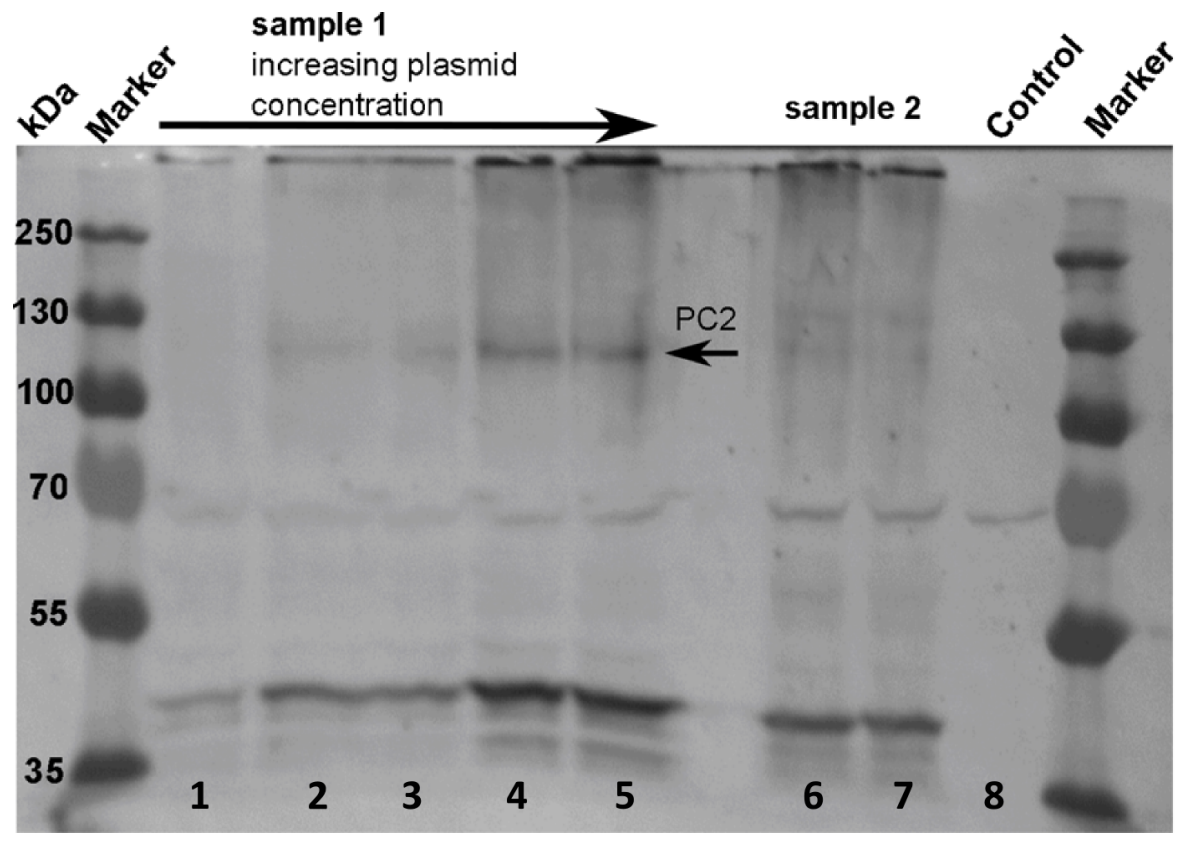

Figure 8.7: Western blot of the PC2 cell-free expression. 1-5 show increasing plasmid concentration $(\mathbf{1} 0.8 \mu \mathrm{g}, \mathbf{2} 1.6 \mu \mathrm{g}, \mathbf{3} 2.4 \mu \mathrm{g}, \mathbf{4} 2.8 \mu \mathrm{g}, \mathbf{5} 4.2 \mu \mathrm{g}), \mathbf{6}$ and $\mathbf{7}$ are samples stored for two weeks at $-80^{\circ} \mathrm{C}$ (plasmid concentrations are $1.6 \mu \mathrm{g}$ and $2.4 \mu \mathrm{g}$ respectively), 8 is the negative control without plasmid added to the reaction mix.

Outer left and right colums are markers indicating the molecular weight.

The Western blot shows a band at $110 \mathrm{kDa}$, which corresponds to polycystin- 2 and is similar to the band observed by $\mathrm{H}$. Cantiello et al using the same expression system [160]. The bands around $50 \mathrm{kDa}$ are probably a result of unfinished translation or unspecific binding of the antibody. Using the recommended plasmid concentration of $1-2 \mu \mathrm{g}$, the bands are very faint, which indicates a low expression level. Raising the plasmid concentration yields a higher intensity, but the unspecific bands at $50 \mathrm{kDa}$ increase as well. In addition, reaction products that were stored at $-80^{\circ} \mathrm{C}$ for two weeks were tested. Here, only very faint bands can be observed at $110 \mathrm{kDa}$, whereas the bands at $50 \mathrm{kDa}$ are more intensive, which indicates that the protein is destroyed when stored too long. Therefore, the expression should occur soon before the reconstitution procedure. 
Polycystin-2 proteins that were expressed in this system were already functionally reconstituted into BLMs by $\mathrm{H}$. Cantiello et al. However, no experiments with the protein could be performed in this work due to a lack of time. Nevertheless, it has been shown that the expression system is suited for expressing the protein and that it can be detected without using audioradiography or modified amino acids so that the success of the expression can be monitored by a simple Western blot, which allows using the same reaction product for analysis (expression control) and experiment (functionality control). 



\section{CHAPTER 9}

\section{Conclusions \& Outlook}

In this thesis, we designed and characterized a lab-on-chip device including a microfabricated chip with an integrated electrode and a sample chamber that allows the application of a variety of experimental techniques on one chip. Four completely separated arrays of pores provide a high throughput, because multiple experiments can be prepared at the same time and measured subsequently.

Three different approaches towards a suitable design were made, including the application of passivation layers ( $\mathrm{SU}-8$ and $\left.\mathrm{SiO}_{2}\right)$ and pure silicon nitride. The approach best suited for lipid bilayer formation turned out to be the preparation on a pure silicon nitride surface. Using AFM and SEM, surface properties such as surface roughness and the pore-shape were determined. Lipid bilayers were prepared via GUV spreading on thiol-gold functionalized and on unfunctionalized porous arrays and imaged with fluorescence microscopy.

Impedance spectroscopy was used to investigate the electrical characteristics of the chip and the integrated electrode and lipid bilayer formation was monitored. The suitability for single ion-channel voltage clamp experiments was tested by incorporating alamethicin into the membranes.

With the end of this thesis, the microporous substrate has been fully characterized electrically and in terms of surface properties, and has been shown to be suited for formation of solvent-free lipid bilayers and methods like fluorescence microscopy, impedance spectroscopy and voltage clamp single channel recordings.

For future projects, many extensions of the experiments shown here are possible. First of all, the already expressed mechanosensitive ion channel polycystin-2, which has never been reconstituted in solvent-free lipid bilayers, can be investigated. As 
it is supposed to react to mechanical stimuli (it forms a flow-sensing complex with polycystin-1), its response towards hydrostatic pressure, which can easily be applied to the lipid bilayer using the in- and outlets of the sample chamber, or even mechanical stimulation with an optical trap, that is included in the setup, can be investigated. This way, the mechanical properties of the lipid bilayer itself can also be investigated. 


\section{Bibliography}

[1] J. F. Danielli and H. Davson. A contribution to the theory of permeability of thin films. Journal of Cellular and Comparative Physiology, 5:495-508, 1935.

[2] S. J. Singer and G. L. Nicolson. The fluid mosaic model of the structure of cell membranes. Science, 175:720-731, 1972.

[3] D. Voet and J. Voet. Biochemistry. John Wiley \& Sons, INC., 2004.

[4] G. van Meer. Cellular lipidomics. The EMBO Journal, 24:3159-3165, 2005.

[5] A. Y. Andreyev, E. Fahy, Z. Guan, S. Kelly, X. Li, J. G. McDonald, S. Milne, D. Myers, H. Park, A. Ryan, B. M. Thompson, Elaine Wang, Y. Zhao, H. A. Brown, A. H. Merrill, C. R. H. Raetz, D. W. Russell, S. Subramaniam, and E. A. Dennis. Subcellular organelle lipidomics in tlr-4-activated macrophages. Journal of Lipid Research, 51:2758-2797, 2010.

[6] A. Shevchenko and K. Simons. Lipidomics: coming to grips with lipid diversity. Nature Reviews Molecular Cell Biology, 11:593-598, 2010.

[7] K. Simon and E. Ikonen. Functional rafts in cell membranes. Nature, 387: 569-572, 1997.

[8] K. Simons and J. L. Sampaio. Membrane organization and lipid rafts. Cold Spring Harbor Perspectives in Biology, 3:1-18, 2011.

[9] A. M. Sokac, C. Schietroma, C. B. Gundersen, and W. M. Bement. Myosin-1c couples assembling actin to membranes to drive compensatory endocytosis. Developemental Cell, 11:629-640, 2006. 
[10] Q. Li, N. Montalbetti, P.Y. Shen, X.Q. Dai, C.I. Cheeseman, E.Karpinski, G. Wu, and H.F. Cantiello andX.Y. Chen. Alpha-actinin associates with polycystin-2 and regulates its channel activity. Human Molecular Genetics, 14:1587-1603, 2005.

[11] S. Demarche, K. Sugihara, T. Zambelli, L. Tiefenauer, and J. Vörö. Techniques for recording reconstituted ion channels. Analyst, 136:1077-1089, 2011.

[12] M. Zagnoni. Miniaturised technologies for the development of artificial lipid bilayer systems. Lab on a Chip, 12:1026-1039, 2012.

[13] A. Granéli, J. Rydström B. Kasemo, and F. Höök. Formation of supported lipid bilayer membranes on sio2 from proteoliposomes containing transmembrane proteins. Langmuir, 19:842-850, 2003.

[14] L. Picas, F. Rico, and S. Scheuring. Direct measurement of the mechanical properties of lipid phases in supported bilayers. Biophysical Journal, 102: L01-L03, 2012.

[15] N. Groulx, H. McGuire, R. Laprade, J.-L. Schwartz, and R. Blunck. Single molecule fluorescence study of the bacillus thuringiensis toxin crylaa reveals tetramerization. The Journal of Biological Chemistry, 286:42274-42282, 2011.

[16] B. R. G. Johnson, R. J. Bushby, J. Colyer, and S. D. Evans. Self-assembly of actin scaffolds at ponticulin-containing supported phospholipid bilayers. Biophysical Journal, 90:L21-L23, 2006.

[17] H. Jung, A. D. Robison, and P. S. Cremer. Detecting protein-ligand binding on supported bilayers by local ph modulation. Journal of the American Chemical Society, 131:1006-1014, 2009.

[18] Q. Xu, W.-C. Lin, R. S. Petit, and J. T. Groves. Epha2 receptor activation by monomeric ephrin-a1 on supported membranes. Biophysical Journal, 101: 2731-2739, 2011. 
[19] A. Alessandrini, H. M. Seeger, T. Caramaschiand, and P. Facci. Dynamic force spectroscopy on supported lipid bilayers: Effect of temperature and sample preparation. Biophysical Journal, 103:38-47, 2012.

[20] S. Aeffner, T. Reusch, B. Weinhausen, and T. Salditt. Energetics of stalk intermediates in membrane fusion are controlled by lipid composition. Proceedings of the National Academy of Sciences of the United States of America, 109:E1609-18, 2012.

[21] C. Scomparin, S. Lecuyer, M. Ferreira, T. Charitat, and B. Tinland. Diffusion in supported lipid bilayers: Influence of substrate and preparation technique on the internal dynamics. European Physical Journal E: Soft Matter, 28: 211-220, 2009.

[22] C. Rossi, S. Doumiati, C. Lazzarelli, M. Davi, F. Meddar, D. Ladant, and J. Chopineau. A tethered bilayer assembled on top of immobilized calmodulin to mimic cellular compartmentalization. PloS One, 6:e19101(1-7), 2011.

[23] M. V. Gudheti, M. Mlodzianoski, and S. T. Hess. Imaging and shape analysis of guvs as model plasma membranes: Effect of trans dopc on membrane properties. Biophysical Journal, 93:2011-2023, 2007.

[24] O. Wesołowska, K. Michalak, J. Maniewska, and A. B. Hendrich. Giant unilamellar vesicles - a perfect tool to visualize phase separation and lipid rafts in model systems. Acta Biochimica Polonica, 56:33-39, 2009.

[25] R. L. Knorr, R. Dimova, and R. Lipowsky. Curvature of double-membrane organelles generated by changes in membrane size and composition. PloS One, 7:e32753(1-14), 2012.

[26] J. C. Stachowiak, C. C. Hayden, and D. Y. Sasaki. Steric confinement of proteins on lipid membranes can drive curvature and tubulation. Proceedings of the National Academy of Sciences of the United States of America, 107: 7781-7786, 2010.

[27] W. Römer, L. Berland, V. Chambon, K. Gaus, B. Windschiegl, D. Tenza, M. R. E. Aly, V. Fraisier, J.-C. Florent, D. Perrais, C.Lamaze, G. Raposo, 
C. Steinem, P. Sens, P. Bassereau, and L. Johannes. Shiga toxin induces tubular membrane invaginations for its uptake into cells. Nature, 450:670678, 2007.

[28] P. M. Vlahovska, R. S. Graciá, S. Aranda-Espinoza, and R. Dimova. Electrohydrodynamic model of vesicle deformation in alternating electric fields. Biophysical Journal, 96:4789-4803, 2009.

[29] T. Yamamoto, S. Aranda-Espinoza, R. Dimova, and R. Lipowsky. Stability of spherical vesicles in electric fields. Langmuir, 26:12390-12407, 2010.

[30] R. Dimova and R. Lipowsky. Lipid membranes in contact with aqueous phases of polymer solutions. Soft Matter, 8:6409-6415, 2012.

[31] S. Aimon, J. Manzi, D. Schmidt, J. A. P. Larrosa, P. Bassereau, and G. E. S. Toombes. Functional reconstitution of a voltage-gated potassium channel in giant unilamellar vesicles. PloS One, 6:e25529(1-11), 2011.

[32] P. Mueller, D. O. Rudin, H. Ti Tien, and W. C. Wescott. Reconstruction of a cell membrane structure in vitro and its transformation into an excitable system. Nature, 194:070-980, 1962.

[33] S. T. White. Ion Channel Reconstitution - Chapter 1: The Physical Nature of Planar Lipid Membranes. Plenum Press, NY, 1986.

[34] L. A. Drachev, A. A. Jasaitis, A. D. Kaulen, A. A. Kondrashin, E. A. Liebermann, I. B. Nemecek, S. A. Ostroumov, A. Y. Semenov, and V. P. Skulachev. Direct measurement of electric current generation by cytochrome oxidase, $\mathrm{h}-$ atpase and bacteriorhodopsin. Nature, 249:321-324, 1974.

[35] M. Montal and P. Mueller. Formation of bimolecular membranes from lipid monolayers and a study of their electrical properties. Proceedings of the National Academy of Sciences of the United States of America, 69:3561-3566, 1972.

[36] T. Gutsmann and U. Seydel. Impact of the glycostructure of amphiphilic membrane components on the function of the outer membrane protein of gram-negative bacteria as a matrix for incorporated channels and a target 
for antimicrobial peptides and proteins. European Journal of Cell Biology, $89: 11-23,2010$.

[37] G. Baaken, M. Sondermann, C. Schlemmer, J. Rühe, and J. C. Behrends. Planar microelectrode-cavity array for high-resolution and parallel electrical recording of membrane ionic currents. Lab on a Chip, 8:938-944, 2008.

[38] A. Hirano-Iwata, K. Aoto, A. Oshima, T. Taira, R. Yamaguchi, Y. Kimura, and M. Niwano. Free-standing lipid bilayers in silicon chips-membrane stabilization based on microfabricated apertures with a nanometer-scale smoothness. Langmuir, 26:1949-1952, 2010.

[39] H. Suzuki, K. Tabata, Y. Kato-Yamada, H. Noji, and S. Takeuchi. Planar lipid bilayer reconstitution with a micro-fluidic system. Lab on a Chip, 4: 502-505, 2004.

[40] B. Le Pioufle, H. Suzuki, K. V. Tabata, H. Noji, and S. Takeuchi. Lipid bilayer microarray for parallel recording of transmembrane ion currents. $A n$ alytical Chemistry, 80:328-332, 2008.

[41] I. Mey, M. Stephan, E. K. Schmitt, M. M. Müller, M. B. Amar, C. Steinem, and A. Janshoff. Local membrane mechanics of pore-spanning bilayers. Journal of the American Chemical Society, 31:7031-7039, 2009.

[42] A. Studer X. Han, H. Sehr, I. Geissbühler, M. Di Berardino, F. K. Winkler, and L. X. Tiefenauer. Nanopore arrays for stable and functional free-standing lipid bilayers. Advanced Materials, 19:4466-4470, 2007.

[43] A. P. Quist, A. Chand, S. Ramachandran, C. Daraio, S. Jin, and R. Lal. Atomic force microscopy imaging and electrical recording of lipid bilayers supported over microfabricated silicon chip nanopores: Lab-on-a-chip system for lipid membranes and ion channels. Langmuir, 23:1375-1380, 2007.

[44] S. Kresák, T. Hianikb, and R. L. C. Naumann. Giga-seal solvent-free bilayer lipid membranes: from single nanopores to nanopore arrays. Soft Matter, 5: 4021-4032, 2009. 
[45] E. K. Schmitt, C. Weichbrodt, and C. Steinem. Impedance analysis of gramicidin d in pore-suspending membranes. Soft Matter, 5:3347-3353, 2009.

[46] F. Heinemann and P. Schwille. Preparation of micrometer-sized free-standing membranes. ChemPhysChem, 12:2568 - 2571, 2011.

[47] M. Bally, K. Bailey, K. Sugihara, D. Grieshaber, J. Vörös, and B. Städler. Liposome and lipid bilayer arrays towards biosensing applications. Small, 6: 2481-2497, 2010.

[48] M. K. Strulson and J. A. Maurer. Microcontact printing for creation of patterned lipid bilayers on tetraethylene glycol self-assembled monolayers. Langmuir, 27:12052-12057, 2011.

[49] T. Yang, E. E. Simanek, and P. Cremer. Creating addressable aqueous microcompartments above solid supported phospholipid bilayers using lithographically patterned poly(dimethylsiloxane) molds. Analytical Chemistry, 72:2587-2589, 2000.

[50] S. Lenhert, C. A. Mirkin, and H. Fuchs. In situ lipid dip-pen nanolithography under water. Scanning, 31:1-9, 2013.

[51] M. Yamada, H. Imaishi, and K. Morigaki. Microarrays of phospholipid bilayers generated by inkjet printing. Langmuir, 29:6404-6408, 2013.

[52] S. Kaufmann, J. Sobek, M. Textorc, and E. Reimhult. Supported lipid bilayer microarrays created by non-contact printing. Lab on a Chip, 11:2403-2410, 2011.

[53] Y. Elani, X. Niud, A. J. DeMello, and O. Ces. Novel technologies for the formation of 2-d and 3-d droplet interface bilayer networks. Lab on a Chip, 12:3514-3520, 2012.

[54] C. Escobedo. On-chip nanohole array based sensing: a review. Lab on a Chip, 13:2445-2463, 2013.

[55] P. V. Ganesan and S. G. Boxer. A membrane interferometer. Proceedings of the National Academy of Sciences of the United States of America, 106: 5627-5632, 2009. 
[56] E. Stava, M. Yu, H. C. Shin, H. Shin, J. Rodrigueza, and R. H. Blick. Mechanical actuation of ion channels using a piezoelectric planar patch clamp system. Lab on a Chip, 12:80-87, 2012.

[57] F. Yi, J. Xu, A. M. Smith, A. N. Parikhc, and D. A. LaVan. Nanofibersupported phospholipid bilayers. Soft Matter, 5:5037-5041, 2009.

[58] G. Chang, R. H. Spencer, A. T. Lee, M. T. Barclay, and D. C. Rees. Crystal structure of escherichia coli mscs,. Science, 282:2220-2226, 1998.

[59] A. Kloda and B. Martinac. Structural and functional similarities and differences between mscmjlr and mscmj, two homologous ms channels of $\mathrm{m}$. jannashii. The EMBO Journal, 8:1888-1896, 2001.

[60] B. Martinac. Mechanosensitive ion channels: molecules of mechanotransduction. Journal of Cell Science, 117:2449-2460, 2004.

[61] E. S. Haswell, R. Phillips, and D. C. Rees. Mechanosensitive channels: What can they do and how do they do it? Structure, 19:1356-1369, 2011.

[62] S. González-Perrett, K. Kon, C. Ibarra, A. E. Damiano, E. Zotta, M. Batelli, P. C. Harris, I. L. Reisin, M. A. Arnaout, and H. F. Caniello. Polycystin-2, the protein mutated in autosomal dominant polycystic kidney desease (adpkd), is a ca2+-permeable nonselective cation channel. Proceedings of the National Academy of Sciences of the United States of America, 98:1182-1186, 2001.

[63] K. Venkatachalam and C. Montell. Trp channels. Annual Review of Biochemistry, 76:387-417, 2007.

[64] R. Sharif-Naeini, J. H. A. Folgering, D. Bichet, F. Duprat, I. Lauritzen, M. Arhatte, M. Jodar, A. Dedman, F. C. Chatelain, U. Schulte, K. Retailleau, L. Loufrani, A. Patel, F. Sachs, P. Delmas, D. J. M. Peters, and E. Honoré. Polycystin-1 and -2 dosage regulates pressure sensing. Cell, 139:587-596, 2009 .

[65] S. M. Nauli and J. Zhou. Polycystins and mechanosensation in renal and nodal cilia. BioEssays, 26:844-856, 2004. 
[66] M Goulian, O. N. Mesquita, D. K. Fygenson, C. Nielsen, O. S. Andersen, and A. Libchaber. Gramicidin channel kinetic under tension. Biophysical Journal, 74:328-337, 1998.

[67] L. R. Opsahl and W. W. Webb. Transduction of membrane tension by the ion channel alamethicin. Biophysical Journal, 66:71-74, 1994.

[68] S. Sukharev and F. Sachs. Molecular force transduction by ion channels diversity and unifying principles. Journal of Cell Science, 125:3075-3083, 2012.

[69] Y. Wu, X.-Q. Dai, Q. Li, C. X. Chen, W. Mai, Z. Hussain, W. Long, N. Montalbetti, G. Li, R. Glynne, S. Wang, H. F. H. F. Caniello, G. Wu, and X.-Z. Chen. Kinesin-2 mediates physical and functional interactions beetween polycystin-2 and fibrocystin. Human Molecular Genetics, 15:32803292, 2006.

[70] X.-Z. Chen, Q. Li Q. Li. Wu, G. Liang, C. J. Lara, and H. F. Caniello. Submembraneous microtubule cytoskeleton: interaction of trpp2 with the cell cytoskeleton. FEBS Journal, 275:4675-4683, 2008.

[71] P. A. Gottlieb, C Bae, and F. Sachs. Gating the mechanical channel piezo1: A comparison between whole-cell and patch recording. Channels, 6:282-289, 2012 .

[72] H. Huang, L. Liang, P. Liu, H. Wei, F. Sachs, W. Niu, and W. Wang. Mechanical effects on katp channel gating in rat ventricular myocytes. PloS One, 8:e63337(1-8), 2013.

[73] M. Köttgen, B. Buchholz, M. A. Garcia-Gonzalez, F. Kotsis, X. Fu, M. Doerken, C. Boehlke, D. Steffl, R. Tauber, T. Wegierski, R. Nitschke, M. Suzuki, A. Kramer-Zucker, G.G. Germino, T. Watnick, J. Prenen, B. Nilius, E. W. Kuehn, and G. Walz. Trpp2 and trpv4 from a polymodal sensory channel complex. Journal of Cell Biology, 182:437-447, 2008.

[74] G. Callejo, J. P. Giblin, and X. Gasull. Modulation of tresk background k+ channel by membrane stretch. PloS One, 8:e64471(1-12), 2013. 
[75] P. Strege, A. Beyder, C. Bernard, R. Crespo-Diaz, A. Behfar, A. Terzic, M. Ackerman, and G. Farrugia. Ranolazine inhibits shear sensitivity of endogenous na + current and spontaneous action potentials in hl-1 cells. Channels, 6:457-462, 2012.

[76] C. C. Häse, A. C. Le Dain, and B. Martinac. Purification and funcitonal reconstitution of the recombinant large mechanosensitive ion channel $(\mathrm{mscl})$ of escherichia coli. The Journal of Biological Chemistry, 270:18329-18334, 1995.

[77] B. Corry, P. Rigby, Z.-W. Liu, and B. Martinac. Conformational changes involved in mscl channel gating measured using fret spectroscopy. Biophysical Journal, 89:L49-L51, 2005.

[78] M. Geissler and Y. Xia. Patterning: Principles and some new developements. Advanced Materials, 16:1249-1269, 2004.

[79] A. Bubendorfer, X. Liu, and A. V Ellis. Microfabrication of pdms microchannels using su-8/pmma moldings and their sealing to polystyrene substrates. Smart Materials and Structures, 16:367-371, 2007.

[80] A del Campo and C. Greiner. Su-8: a photoresist for high-aspect-ratio and 3d submicron lithography. Journal of Micromechanics and Microengineering, 17:R81-R95, 2007.

[81] A. Douvas, P. Argitis, K. Misiakos, D. Dimotikali, P. S. Petrou, and S. E. Kakabakos. Biocompatible photolithographic process for the patterning of biomolecules. Biosensors and Bioelectronics, 17:269-278, 2002.

[82] J. L. Dektar and N. P. Hacker. Photochemistry of triarylsulfonium salts. Journal of the American Chemical Society, 112:6004-6015, 1990.

[83] MicroChem Inc. spincoating photoresist, August 2013. www. microchemicals.eu/technical_information.

[84] T. A. Anhoj, A. M. Jorgensen, D. A Zauner, and J. Hübner. The effect of soft bake temperature on the polymerization of su- 8 photoresist. Journal of Micromechanics and Microengineering, 16:1819-1824, 2006. 
[85] R. Daunton, A. J. Gallant, and D. Wood. Manipulation of exposure dose parameters to improve production of high aspect ratio structures using su- 8 . Journal of Micromechanics and Microengineering, 22:075016 (8pp), 2012.

[86] C.-M. Cheng and R.-H. Chen. Development behaviours and microstructure quality of downward-development in deep x-ray lithography. Journal of Micromechanics and Microengineering, 11:692-696, 2001.

[87] Y.-J. Chuang, F.-G. Tseng, and W.-K. Lin. Reduction of diffraction effect of uv exposure on su- 8 negative thick photoresist by air gap elimination. Microsystem Technologies, 8:308-313, 2002.

[88] R. Fabian Pease and Stephen Y. Chou. Lithography and other patterning techniques for future electronics. Proceedings of the IEEE, 96:248-270, 2008.

[89] I. Malik, V. Hazari, K. Monahan, M. Hankinson, M. Adel, M. Liesching, and E. Charrier. Immersion lithography - process and control challenges. Yield Management Solutions, 9:7-17, 2007.

[90] M. J. Madou. Fundermentals of Microfabrication. CRC Press, Boca Raton, New York, 1997.

[91] Oxford Instruments. Reactive ion etch (rie), August 2013. http://www.oxford-instruments.com/products/etching-deposit ion-and-growth/plasma-etch-deposition/rie.

[92] Oxford Instruments. Plasma enhanced chemical vapour deposition (pecvd), August 2013. http://www.oxford-instruments.com/product s/etching-deposition-and-growth/plasma-etch-deposition/pecvd.

[93] X. Peng, A. Matthews, and S. Xue. Plasma-based processes and thin film equipment for nano-scale device fabrication. Journal of Materials Chemistry, 46:1-37, 2011.

[94] E. Sardella, F. Intranuovo, P. Rossini, M. Nardulli, R. Gristina, R. d'Agostino, and P. Favia. Plasma enhanced chemical vapour deposition of nanostructured fluorocarbon surfaces. Plasma Processes and Polymers, 6: S57-S60, 2009. 
[95] K. L. Choy. Chemical vapour deposition of coatings. Progress in Materials Science, 48:57-170, 2003.

[96] Oerlikon Leybold Vacuum. UNIVEX 350 Experimentelle VakuumBeschichtungsanlage Gebrauchsanleitung.

[97] H. Seidel, L. Csepregi, A. Heuberger, and H. Baumgärtel. Anisotropic etching of crystalline silicon in alkaline solutions i. orientation dependence and behavior of passivation layers. Journal of the Electrochemical Society, 137: 3612-3626, 1990.

[98] H. Seidel, L. Csepregi, A. Heuberger, and H. Baumgärtel. Anisotropic etching of crystalline silicon in alkaline solutions - ii. influence of dopants. Journal of the Electrochemical Society, 137:3626-3623, 1990.

[99] I. Zubel. Silicon anisotropic etching in alkaline solutions ii - on the influence of anisotropy on the smoothness of etched surfaces. Sensors and Actuators, A: Physical, 70:260-268, 1998.

[100] I. Zubel and I. Barycka. Silicon anisotropic etching in alkaline solutions i. the geometric description of figures developed under etching si (100) various solutions. Sensors and Actuators, A: Physical, 70:250-259, 1998.

[101] K. Sato, M. Shikida, Y. Matsushima, T.i Yamashiro, K. Asaumi, Y. Iriye, and M. Yamamoto. Characterization of orientation-dependent etching properties of single-crystal silicon: effects of koh concentration. Sensors and Actuators, A: Physical, 64:87-93, 1998.

[102] K. Sato, M. Shikida, T. Yamashiro, M. Tsunekawa, and S. Ito. Roughening of single-crystal silicon surface etched by koh water solution. Sensors and Actuators, 73:122-130, 1999.

[103] M. H. Yun, V. A. Burrows, and M. N. Kozicki. Analysis of koh etching of $<100>$ silicon on insulator for the fabrication of nanoscale tips. Journal of Vacuum Science \& Technology, B, 16:2844-2848, 1998.

[104] G. Binnig, C. F. Quate, and C. Gerber. Atomic force microscope. Physical Review Letters, 56:930-934, 1986. 
[105] J. K. H. Hörber and M. J. Miles. Scanning porbe evolution in biology. Science, 302:1002-1005, 2003.

[106] Olympus Corp. Olympus micro cantilever, August 2013. http://probe. olympus-global. com.

[107] F. Krumeich and Laboratory of Inorganic Chemistry ETH Zurich. Properties of electrons, their interactions with matter and applications in electron microscopy, August 2013. http://www.microscopy.ethz.ch/downloads/Int eractions.pdf.

[108] B. Voutou, E.-C.i Stefanaki, and K. Giannakopoulos. Physics of advanced materials winter school 2008: Electron microscopy: The basics, 2008. http: //www.mansic.eu/documents/PAM1/Giannakopoulos1.pdf.

[109] FEI. An introducton to electricon microscopy, August 2013. http://www.fei.com/resources/student-learning/introduction-t o-electron-microscopy/resources .aspx.

[110] MicroChem Inc. Su-8 2000 permanent epoxy negative photoresist, processing guidelines for su-8 2000.5, su-8 2002, su-8 2005, su-8 2007, su-8 2010 and su-8 2015, August 2013. www.microchem.com/pdf/SU-82000DataSheet2000_5t hru2015Ver4.pdf.

[111] Virginia Semiconductor. The general properties of si, ge, sige, sio2 and si3n4. Technical report, Virginia Semiconductor, 2002.

[112] S. L. Tao, K. C. Popat, J. J. Norman, and T. A. Desai. Surface modification of su- 8 for enhanced biofunctionality and nonfouling properties. Langmuir, 24:2631-2636, 2008.

[113] F. Walther, P. Davydovskaya, S. Zurcher, M. Kaiser, H. Herberg, A. M. Gigler, and R. W. Stark. Stability of the hydrophilic behavior of oxygen plasma activated su-8. Journal of Micromechanics and Microengineering, $17: 524,2007$.

[114] E. Bonaccurso and K. Graf. Nanostructuring effect of plasma and solvent treatment on polystyrene. Langmuir, 20:11183-11190, 2004. 
[115] C. Teichert, J.F. MacKey, D.E. Savage, M.G. Lagally, M. Brohl, and P. Wagner. Comparison of surface roughness of polished silicon wafers measured by light scattering topography soft-x-ray scattering and atomic-force microscopy. Applied Physics Letters, 96:2346-2348, 1995.

[116] H. Huang, A. Suvorova, K. J. Wincherster, J. M. Dell, and L. Faraone. Characteristics of low temperature pecvd silicon nitride for mems structural materials. International Journal of Modern Physics B, 20:3799-3804, 2006.

[117] S.J. Wilk, L. Petrossian, M. Goryll, T.J. Thornton, S.M. Goodnick, J.M. Tang, and R.S. Eisenberg. Integrated electrodes on a silicon based ion channel measurement platform. Biosensors and Bioelectronics, 23:183-190, 2007.

[118] T. Mach, C. Chimerel, J. Fritz, N. Fertig, M. Winterhalter, and C. Fütterer. Miniaturized planar lipid bilayer: increased stability, low electric noise and fast fluid perfusion. Analytical and Bioanalytical Chemistry, 390:841-846, 2008 .

[119] J. D. Uram, K. Ke, and M. Mayer. Noise and bandwidth of current recordings from submicrometer pores and nanopores. ACS Nano, 5:857-872, 2008.

[120] M. I. Angelova and D. S. Dimitrov. Liposome electroformation. Faraday Discussions of the Chemical Society, 81:303-311, 1986.

[121] D.S. Dimitrov and M.I. Angelova. Lipid swelling and liposome formation mediated by electric fields. Bioelectrochemistry and Bioenergetics, 19:232226, 1988.

[122] L.A. Bagatolli ans T. Parasassi and E. Gratton. Giant phospholipid vesicles: comparison among the whole lipid sample characteristics using different preparation methods: A two photon fluorescence microscopy study. Chemistry and Physics of Lipids, 105:135-147, 2000.

[123] M. Kocun, T. D. Lazzara, C. Steinem, and A. Janshoff. Preparation of solvent-free, pore-spanning lipid bilayers: Modeling the low tension of plasma membranes. Langmuir, 27:7672-7680, 2011. 
[124] H. G. L. Coster and J. R Smith. The organisation of biomolecular lipid membranes a study of hte low frequency maxwell-wagner impedance dispersion. Biochimica et Biophysica Acta, 373:151-164, 1974.

[125] P. M. Gomadam and J. W. Weidner. Analysis of electrochemical impedance spectroscopy in proton exchange membrane fuel cells. International Journal of Energy Research, 29:1133-1151, 2005.

[126] F. Fabregat-Santiagoa and J. Bisquert. Impedance spectroscopy study of dyesensitized solar cells with undoped spiro-ometad as hole conductor. Journal of Applied Physics, 100:034510(1-7), 2006.

[127] F. Wenger and J. Galland. Analysis of local corrosion of large metallic structures or reinforced concrete structures by electrochemical impedance spectroscopy (eis). Electrochimica Acta, 35:1573-157, 1990.

[128] D. Kloß, M. Fischer, A. Rothermel, J. C. Simon, and A. A . Robitzki. Drug testing in $3 \mathrm{~d}$ in virto tissues trapped on a microcavity chip. Lab on a Chip, 8:879-884, 2008.

[129] N.M.M. Pires, T. Dong, Z. Yang, N. Høivik, and X. Zhao. A mediator embedded micro-immunosensing unit for electrochemical detection on viruses within physiological saline media. Journal of Micromechanics and Microengineering, 21:115031(1-11), 2011.

[130] C. Chen, A. Ganguly, C. Wang, C. Hsu, S. Chattopadhyay, Y. Hsu, Y. Chen, and L. Chen. Label-free dual sensing of dna molecules using gan nanowires. Analytical Chemistry, 81:36-42, 2009.

[131] X. Cheng, Y. Liu, D. Irimia, U. Demirci, L. Yang, L. Zamir, W. R. Rodríguez, M. Toner, and R. Bashir. Cell detection and counting trough cell lysate impedance spectroscopy in microfluidic devices. Lab on a Chip, 7:746-755, 2007.

[132] Isaak O. K-Owino and Omowunmi A. Sadik. Impedance spectroscopy: A powerful tool for rapid biomolecular screening and cell culture monitoring. Electro, 23:2101-2113, 2005. 
[133] C. Xiao and J. H.T. Luong. Assessment of cytotoxicity by emerging impedance spectroscopy. Toxicology and Applied Pharmacology, 206:102-112, 2005 .

[134] E. K. Schmitt. Hochohmige porenüberspannende Lipidmembranen: Elektrochemische Untersuchungen zur Aktivität von Gramicidin und Bacteriorhodopsin. PhD thesis, Georg-August University Göttingen, 2009.

[135] S. Alonso-Romanowski, L. M. Gassa, and J. R. Vliche. An investigation by eis of gramicidin channels in bilayer lipid membranes. Electrochimica Acta, 40:1561-1567, 1995.

[136] V. Atanasov, N. Knorr, R. S. Duran, S. Ingebrandt, A. Offenhäusser, and I. Köper W. Knoll. Membrane on a chip: A functional thethered lipid bilayer membrane on silicon oxide surfaces. Biophysical Journal, 89:1780-1788, 2005.

[137] F. Lisdat and D. Schäfer. The use of electrochemical impedance spectroscopy for biosensing. Analytical and Bioanalytical Chemistry, 391:1555-1567, 2008.

[138] Gamry Instruments. Basics of electrochemical impedance spectroscopy, August 2013. http://www.gamry.com/application-notes/EIS/.

[139] S. Ohki. Dielectric constant and refractive index of lipid bilayers. Journal of Theoretical Biology, 19:97-115, 1968.

[140] G. Gramse, A. Dols-Perez, M. A. Edwards, L. Fumagalli, and G. Gomila. Nanoscale measurement of the dielectric constant of supported lipid bilayers in aqueous solutions with electrostatic force microscopy. Biophysical Journal, 109:1257-1262, 2013.

[141] C. G. Malmberg and A. A. Maryott. Dielectric constant of water. Journal of Research of the National Bureau of Standards, 56:369131(1-8), 1956.

[142] R. Benz, O. Fröhlich, P. Läger, and M. Montal. Electrical capacity of black lipid films and of lipid bilayers made from monolayers. Biochimica et Biophysica Acta, 394:323-334, 1975. 
[143] K. S. Cole and R. H. Cole. Dispersion and absorption in dielectrics. i. alternating current characteristics. Journal of Chemical Physics, 9:341-351, 1941.

[144] C. S. Hsu and F. Mansfield. Concerning the conversion of the constant phase element parameter y0 into a capacitance. Corrosion, 57:474-478, 2001.

[145] B. Hirschorn, M. E. Orazem, B. Tribollet, V. Vivier, I. Frateur, and M. Musianid. Constant-phase-element behavior caused by resistivity distributions in films. Journal of the Electrochemical Society, 157:C452-C457, 2010.

[146] R. S. Rogers. Resources for electrochemistry - fitting eis data - diffusion elements - warburg, August 2013. http://www.consultrsr. com/resources/eis/diffusion.htm.

[147] E. Warburg. Über das verhalten sogenannter unpolarisierbarer elektroden. Annual Review of Physical Chemistry, 67:493-499, 1899.

[148] J.E.B. Randle. Kinetics of rapid electrode reactions. Transactions of the Faraday Society, 48:937-950, 1952.

[149] V. B. Sundaresan, S. A. Sarles, and D. J. Leo. Characterization of porous substrates for biochemical energy conversion devices. Proceedings of SPIE, 6928:69280K(1-11), 2008.

[150] B. J. Polk, A. Stelzenmuller, G. Mijares, W. MacCrehan, and M. Gaitan. $\mathrm{Ag} /$ agcl microelectrodes with improved stability for microfluidics. Sensors and Actuators B, 114:239-247, 2006.

[151] R. Benz, O. Fröhlich, P. Läuger, and M. Montal. Electric capacity of black lipd films and of lipid bilayers made from monolayers. Biochimica et Biophysica Acta, 394:323-334, 1975.

[152] E. N. Ervin and H. S. White. Alternating current impedance imaging of membrane pores using scanning electrochemical microscopy. Analytical Chemistry, $77: 5564-5569,2005$.

[153] J. E. Hall. Access resistance of a small circular pore. Journal of General Physiology, 66:531-532, 1975. 
[154] A. L. Hodgkin, A. F. Huxley, and B. Katz. measurement of current voltage relations in the membrane of the giant axon of loligo. Journal of Physiology, 115:424-448, 1952.

[155] K. S. Cole and A. L. Hodgkin. Membrane and protoplasm resistance in the squid giant axon. Journal of General Physiology, 22:671-687, 1939.

[156] M.S.P. Sansom. The biophysics of peptide models of ion channels. Progress in Biophysics and Molecular Biology, 55:139-235, 1991.

[157] D. Peter Tieleman, Berk Hess, and Mark S. P. Sansom. Analysis and evaluation of channel models: Simulations of alamethicin. Biophysical Journal, 83:3293-2407, 2002.

[158] S. L. Keller, S. M. Bezrukov, S. M. Gruner, M. W. Tate, I. Vodyanoy, and V. A. Parsegian. Probability of alamethicin conductance states varies with nonlamellar tendency of bilayer phospholipids. Biophysical Journal, 65:2327, 1993.

[159] T. Mochizuki, G. Wu, T. Hayashi, S. L. Xenophontos, B. Veldhuisen, J. J. Saris, D. M. Reynolds, Y. Cai, P. A. Gabow, A. P. Pierides, W. J. Kimberling, M. H. Breuning, C. C. Deltas, and D. J. M. Peters. Somlo. Pkd2, a gene for polycystic kidney disease that encodes an integral membrane protein. Science, 272:1339-1342, 1996.

[160] G. M. Xu, S. González-Perrett, M. Essafi, G. A. Timpanoro, N. Montalbetti, M. A. Arnaout, and H. F. Cantiello. Polycystin-1 activates and stabilzes the polycystin-2 channel. The Journal of Biological Chemistry, 278:1457-1452, 2003.

[161] S. González-Perrett, M. Batelli, K. Kim, M. Essafi, G. A. Timpanaro, N. Moltabetti, I. L. Reisin, M. A. Arnaout, and H. F. Caniello. Voltage dependence and ph regulation of human polycystin-2-mediated cation channel activity. The Journal of Biological Chemistry, 277:24959-24966, 2002.

[162] A. Giamarchi, F. Padilla, B. Coste, M. Raoux, M. Crest, and E. Honoré. The versatile nature of the calcium-permeable cation channel trpp2. EMBO Reports, 7:787-793, 2006. 
[163] Y. Cai, Maeda, A. Cedzich, V. E. Torres, G. Wu, T. Hayashi, T. Mochizuki, J. H. Park, R. Witzgall, and S. Somlo. Identification and characterization of polycystin-2, the pdkd2 gene product. The Journal of Biological Chemistry, 274:28557-28565, 1999.

[164] P. Koulen, Y. Cai, A. Geng, Y. Maeda, S. Nishimura, R. Witzgall, B. E. Ehrlich, and S. Somlo. Polycystin-2 is an intracellular calcium release channel. Nature Cell Biology, 4:191-197, 2002.

[165] N. G. Gantoso, L. Cebotaru, and W. B. Guggino. Polycystin-1, 2, and stim1 interact with ip3r to modulate er ca2 + release through the pi3k/akt pathway. Cellular Physiology and Biochemistry, 27:715-726, 2011.

[166] C. Qiu and J. Yu. The function of polycystin-1 and polycystin-2 in cardiovascular system. Journal of Cardiovascular Diseases \& Diagnosis, 1:1000110(16), 2013 .

[167] M. del Rocío Cantero and H. F. Cantiello. Calcium transport and local pool regulate polycystin-2 (trpp2) function in human syncytiotrophoblast. Biophysical Journal, 105:365-375, 2013.

[168] A. P. Stewart, G. D. Smith, R. N. Sandford, and J. M. Edwardson. Atomic force microscopy reveals the alternating subunit arrangement of the trpp2trpv4 heterotetramer. Biophysical Journal, 99:790-797, 2010.

[169] T. Kobori, G. D. Smith, R. N. Sandford, and J. M. Edwardson. The transient resceptor potential channels trpp2 and trpc1 form a heterotetramer with a 2:2 stoichiometry and an alternating subunit arrangement. Journal of Biological Chemistry, 284:35507-35513, 2009.

[170] S. Yoshiba, H. Shiratori, I. Y. Kuo, A. Kawasumi, K. Shinohara, S. Nonaka, Y. Asai, G. Sasaki, J. A. Belo, H. Sasaki, J.Nakai, B. Dworniczak, B. E. Ehrlich, P. Pennekamp, and H. Hamada. Cilia at the node of mouse embryos sense fluid flow for left-right determination via pkd2. Science, 338:226-231, 2012 .

[171] S. M. Nauli, F. J. Alenghat, Y. Luo, W. Williams, P. Vassilev, X. Li, A. E. H. Elia, W. Lu, E. M. Brown, J. Quinn, D. E. Ingber, and J. Zhou. Polycystins 
1 and 2 mediate mechanosensation in the primary cilium of kidney cells. Nature Genetics, 33:129-137, 2003.

[172] Q. Li, X.-Q. Dai, P. Y. Shen, H.F. Cantiello, E. Karpinski, and X.-Z. Chen. A modified mammalian tandem affintiy purification procedure to prepare fuctional polycystin-2 channel. FEBS Letters, 576:231-236, 2004.

[173] T. Hayashi, T. Mochizuki, D. M. Reynolds, G. Wu, Y. Cai, and S. Somlo. Characterization of the exon structure of the polycystic kidney disease 2 gene (pkd2). Genomics, 44:131-136, 1997.

[174] Promega. Protein expression, August $2013 . \quad$ http://www . promega.de/resources/product-guides-and-selectors/prot ocols-and-applications-guide/protein-expression/?origUrl= http $\% 3 a \% 2 f \% 2$ f www . promega. com $\% 2$ fresources $\% 2$ fproduct -guides-and-selectors $\% 2$ fprotocols-and-applications-guide\% 2 fprotein-expression $\% 2 f$. 



\section{List of Figures}

2.1 The fluid mosaic model as proposed by S. J. Singer and G. L. Nicolson, adapted from $[2] . \ldots \ldots \ldots \ldots$. . . . . . . . . . . . 4

2.2 Different techniques for biological membrane models are (clockwise) the Black Lipid Membrane (BLM), Giant Unilamellar Vesicles (GUVs), solid supported membranes and lipid bilayer on porous supports (microBLM or nanoBLM) . . . . . . . . . . . . . . 7

3.1 The microporous substrate designed and fabricated in this work. Details on the fabrication process are given in the text. . . . . . . 15

3.2 In photolithographic processes a photopolymer is exposed to UVlight through a mask defining the desired structures. The exposed areas are removed after exposure when positive tone-photoresist is used, whereas negative-tone photoresists remains on the substrate. . 17

3.3 The SU-8 monomer contains eight epoxy groups which react during the crosslinking process. . . . . . . . . . . . . . 18

3.4 Overview of the SU-8 processing steps. . . . . . . . . . . . 18

3.5 Reaction chambers for Plasma Enhanced Chemical Vapour Deposition (left) and Reactive Ion Etching (right) [91, 92] . . . . . . . . . 24

3.6 The etch rates in the $\mathrm{KOH}$ etch porcess of single crystal silicon depend on the crystal layer. The (111) layer is etched significantly slower than the $(100)$ layer. . . . . . . . . . . . . . . 28

3.7 The $\mathrm{KOH}$ etch of the silicon nitride membrane at three different stages of the etch process. The pure silicon nitride membrane appears at the end of the etch porcess as a flat layer, whereas the silicon is structured due to the crystal layer dependent etch process. 30 
3.8 Porous arrays with $\mathrm{N}=9(\mathbf{A})$ and $\mathrm{N}=900$ pores $(\mathbf{B}) \ldots \ldots 31$

3.9 Cross sections of the three different approaches for the substrate fabrication. In $\mathbf{A}$ the SU-8 photoresist remains on the substrate, in B a silicon dioxide layer for passivation was deposited, resulting in conically shaped pores. $\mathbf{C}$ shows the final approach, resulting in a low surface roughness and cylindrically shaped pores (chapter 4).

3.10 In step 1 of the fabrication process the silicon wafer is diced (1) and a PECVD silicon nitride layer is deposited on both sides (2). In step 2 the pore areas are patterned $(\mathbf{3})$ and the silicon nitride is removed from four squares on the back side (4). The bare silicon is etched with $\mathrm{KOH}(30 \%)$ so that four silicon nitride membranes remain on the front side $(5) \ldots \ldots \ldots \ldots \ldots \ldots$

3.11 In step 3 the pores $(1 \mu \mathrm{m}$ in diameter) are patterned using SU-8 photoresist (1) and etched in RIE process (2). The photoresist is delaminated from the substrate with $\mathrm{KOH}$ solution (3). . . . . . . 36

3.12 In step 3 the integrated electrode $(1 \mathrm{~mm}$ in width and $150 \mathrm{~nm}$ in height) is deposited on the substrate by thermal evaporation of silver. For better adhesion a layer of titanium (10 $\mathrm{nm}$ in height) is deposited before by electron beam evaporation.

3.13 Alternative methods for silver electrode generation are lift-off processes (left) or the use of photoresist protection layers (right). . . .

3.14 Silver electrodes obtained by using a photoresist protection layer (A) and a mechanical aluminum mask $($ B $) \ldots \ldots \ldots \ldots$

4.1 Atomic force microscopy provides detailed information on the surface roughness, but when imaging holes with a large diameter, artifacts can occur. . . . . . . . . . . . . . . . 39

4.2 The feret-diameter and the roundness of the pores was estimated by a pixel analysis of brightfield images A. A threshold was adjusted to the pores and the area was calculated B. . . . . . . . . . . . 40

4.3 Working principle of the AFM. A sharp tip scans across the surface of a specimen. The changes in topography are detected by a laser beam that is reflected onto a quadrant photo diode. . . . . . . . . 
4.4 Electrons can interact elastically and inelastically with matter. Elastic interaction leads to elastic scattering, the direct beam and backscattering. Inelastic interactions include the generation of secondary electron, Auger electrons and X-rays. . . . . . . . . . . . . . 43

4.5 SU-8 photoresist can be rendered hydrophilic by an acid-catalyzed ring-opening reaction of the epoxide ring (A) which makes lipid bilayer formation possible on solid supports $($ B) . . . . . . . . . .

4.6 AFM and SEM images SU-8-based approach, which have properly etched pores (A, B), but a high surface roughness $(\mathbf{C})$. . . . . . 46

4.7 AFM and SEM images of the the silicon dioxide coated substrates that have a low surface roughness $(\mathbf{C})$, but cone-shaped pores $(\mathbf{A}, \mathbf{B}) .47$

4.8 AFM and SEM images of the silicon nitride based substrate type with cylindrical pores (A, B) and low surface roughness $\mathbf{C}$ which was used in the experiments. . . . . . . . . . . . . . . . 48

5.1 Photography (by Christopher Battle) of the setup including the microscope body 2 , part of the faraday cage (2) surrounding microscope and electrophysiology parts, lasers for optical trapping (4), and illumination (3). The sample can be illuminated from below with and LED placed under the xyz-stage (5). All associated optics for the optical trap are on an aluminum platform (6) and the connections to single channel amplifier and impedance spectrometer are on the left side (7). The micromanipulator for patch-clamp experiments is placed behind the microscope body (8) . . . . . . 51

5.2 Setup for voltage clamp single channel recordings consisting of the sample chamber and the pre-amplifier that are enclosed in a Faraday cage, the main-amplifier, the $\mathrm{AD} / \mathrm{DA}$ converter and the computer with the acquisition software. . . . . . . . . . . . . 52

5.3 The sample chamber consists of three parts, with separated electrolyte compartments in the lower part for each of the arrays and a common compartment on the top. The chamber can be sealed tightly by a screw-cap. . . . . . . . . . . . . . . . . 53 
5.4 The chamber for the electroformation of GUVs consists of two ITOcoated glass slides separated by a silicone spacer, and copper tape for the electrical connection. . . . . . . . . . . . . . . . . 54

5.5 Lipid bilayers on unfunctionalized silicon nitride. The lipid bilayer was prepared from DOPC vesicles doped with $0.5 \mathrm{~mol} \%$ OregonGreen 488 DHPE. . . . . . . . . . . . . . . . . 56

5.6 Lipid bilayers on gold/thiol functionalized substrates. (A) was taken with a 100x water-dipping objective (LUMPlanFL W, Olympus Corp., Tokyo, JP), (B) with a $63 \mathrm{x}$ water immersion objective (C-Apochromat, Carl Zeiss AG, Jena, GE ). Both are DOPC lipid bilayers doped with 1 mol\% $\beta$-BODIPY-500/510-C 12 -HPE. . . . . . . . . . 56

6.1 In a vector diagram the modulus $|Z|(\omega)$ of the impedance is the vector between the imaginary and the real part of the impedance. The phase angle is the the angle between absolute value and real part of the impedance. . . . . . . . . . . . . . . . 6 61

6.2 The figure shows Nyquist $\mathbf{A}$ and Bode plots $\mathbf{B}$ of an $R_{e l}\left(R_{1} \mathrm{C}_{1}\right)$ circuit $\mathbf{C}$. The parameters for the circuit elements are: $R_{e l}=100 \Omega$, $R_{1}=10^{6} \Omega$ and $C_{1}=10^{-6} \mathrm{~F} \ldots \ldots \ldots \ldots$

6.3 A pseudo-linear current-voltage relation is created by using only a small voltage amplitude [138]. . . . . . . . . . . . . . .

6.4 The lipid bilayer can be modeled by a resistance and a capacitor in parallel. The hydrophobic core acts as a resistor and as a dielectric layer between the two electrolyte compartments. . . . . . . . . . 64

6.5 In a Bode plot, the CPE element in parallel to a resistor appears as a depressed semi circle compared to an ideal capacitance. . . . . . . 66

6.6 In a Bode plot the Warburg element appears as streight line at an angle of $45^{\circ} \ldots \ldots \ldots \ldots \ldots$. . . . . . . . . . . . 68

6.7 Impedance spectra fitted with the equivalent circuit proposed by Kresak et al. (A), impedance data are displayed as white circles and the fit as a solid line. . . . . . . . . . . . . . 70

6.8 Impedance spectra fitted with the equivalent circuit proposed by Tiefenauer et al. (B), impedance data are displayed as white circles and the fit as a solid line. . . . . . . . . . . . . . . 71 
6.9 Impedance spectra were fitted with an $\mathrm{R}(\mathrm{RC})$ circuit shown in $\mathbf{C}$. Impedance data are displayed as white circles and the fit as a solid line. . . . . . . . . . . . . . . . . . . 72

6.10 The spectra of integrated (white circles) and external electrodes (black squares) differ in the low frequency part. . . . . . . . . . 73

6.11 The impedance spectra of substrates with integrated electrodes show an additional R/CPE element. . . . . . . . . . . . 75

6.12 Impedance spectra of lipid bilayers recorded with external (white circles) and integrated electrodes (black squares) from the same experiment cannot be distinguished. Both were fitted with an $\mathrm{R}(\mathrm{RC})$ circuit (red solid line). . . . . . . . . . . . . . 77

6.13 The round edges of the pores lead to a an entrance radius $r_{\text {entry }}$ that is higher than the actual pore radius $r_{\text {pore }} \ldots \ldots \ldots \ldots . \ldots 79$

6.14 The capacitance can be calculated from the current response of a triangular voltage pulse with a slope of $1 \mathrm{~V} / \mathrm{s} . \ldots . . . . .$.

6.15 The current response to a triangular voltage pulse at a high ohmic resistance. . . . . . . . . . . . . . . 81

7.1 Example single-channel recording with four conductance levels (blue lines 0-3) set with the data anaysis software Clampfit. . . . . . 85

7.2 Representative single channel traces obtained for alamethicin in DOPC lipid bilayers in $1 \mathrm{M} \mathrm{KCl}$ solution. . . . . . . . . . . . . . . 87

7.3 The conductance histogram from all single channel events recorded at $100 \mathrm{mV}$ show three main conductance levels at $(176 \pm 7) \mathrm{pS},(486 \pm 8) \mathrm{pS}$ and $(837 \pm 11) \mathrm{pS}$ and few higher conductance levels at $(1448 \pm 77) \mathrm{pS}$ $(\mathrm{n}=6)$ and $(3697 \pm 206) \mathrm{pS}(\mathrm{n}=5) \ldots \ldots \ldots . \ldots . \ldots 88$

8.1 Schematic structure of polycystin-2, adapted from [162] . . . . . . . 90

8.2 The vector pc-DNA3.1/HisC containing polycystin-2 between position 484 and the T7 promoter was used for the cell-free expression. 
8.3 Result of the restriction digest of the polycystin-2 containing vector pc-DNA3.1/HisC before the digest (column 2, pure), after digest with Nde (column 3, Nde), after digest with Xho (column 4, Xho) and after digest with both Nde and Xho (column 5, Nde + Xho). Column 1 shows a marker for orientation. . . . . . . . . . . . . 94

8.4 Dot blot for the determination of a suitable secondary antibody and dilution of the primary antibody. I-III are secondary antibodies: anti-rabbit antibody 2005, anti-rabbit antibody 2009 and anti-mouse antibody. The dilutions of the primary antibody are $(\mathbf{A} 1 / 200, \mathbf{B}$ 1/400, C 1/100, D 1/1000). For positive control two anti-rabbit antibodies $\mathbf{E}$ and $\mathbf{F}$ were used, for negative control anti-mouse antibody G. . . . . . . . . . . . . . . . . 95

8.5 Scheme for the cell-free expression of polycystin-2 [174] . . . . . . . 96

8.6 SDS-page obtained from the whole reaction mix of the cell-free expression. Column one shows the result of the reaction with $3 \mu \mathrm{g}$ DNA and column two the negative control without DNA. . . . . . 97

8.7 Western blot of the PC2 cell-free expression. 1-5 show increasing plasmid concentration (1 $0.8 \mu \mathrm{g}, \mathbf{2} 1.6 \mu \mathrm{g}, \mathbf{3} 2.4 \mu \mathrm{g}, \mathbf{4} 2.8 \mu \mathrm{g}, \mathbf{5}$ $4.2 \mu \mathrm{g}$ ), $\mathbf{6}$ and $\mathbf{7}$ are samples stored for two weeks at $-80^{\circ} \mathrm{C}$ (plasmid concentrations are $1.6 \mu \mathrm{g}$ and $2.4 \mu \mathrm{g}$ respectively), 8 is the negative control without plasmid added to the reaction mix. Outer left and right colums are markers indicating the molecular weight. . . . . . . 98 


\section{List of Tables}

3.1 Process parameters for photolithography fabricaiton steps . . . . . 23

3.2 Process parameters for RIE processes. . . . . . . . . . . . . . 27

3.3 Process parameters for PECVD silicon nitride deposition. . . . . . . 27

6.1 Parameters of the fitting results for all spectra . . . . . . . . 73

6.2 Parameters of the fitting results for all spectra . . . . . . . . 75

8.1 Components and volumes for the cell-free expression of polycystin-2. 96 



\title{
Appendix
}

\section{Abbreviations \& acronyms}

\author{
ADPKD Autosomal Dominat Polycystic Kidney Disease \\ AFM Atomic Force Microscopy \\ BLM Black Lipid Membrane \\ CPE Constant Phase Element \\ CPEO3 Cholesterylpolyethylenoxythiol \\ DOPC 1,2-Dioleoyl-sn-glycero-3-phosphocholine \\ EDTA Ethylenediaminetetraacetate \\ ER Endoplasmic Reteculum \\ GUV Giant Unilamellar Vesicle \\ ITO Indium-tin-oxide \\ MEMS Microelectromechanical Systems \\ MSC Mechanosensitive Ion Channel \\ RIE Reactive Ion Etch \\ PC2 Polycystin-2 \\ PDMS Poly(dimethylsiloxane) \\ PEB Post Exposure Bake \\ PECVD Plasma Enhanced Chemical Vapor Deopsition \\ PGMEA propylene glycol monomethyl ether acetate \\ PM Plasma Membrane \\ PMMA Poly(methyl methacrylate) \\ SEM Scanning Electron Microscopy \\ TEM Transmission Electron Microscopy \\ TRP Transient Receptor Potential
}




\section{Chemicals \& materials}

Adhesive Copper tape

$\beta$-BODIPY-500/510-C12-HPE

Cantilever

DOPC

EDTA

Gold

ITO glass slides

MES

$\mathrm{KCl}$

$\mathrm{KOH}$

Oregon-Green 488 DHPE

PDMS (SYLGARD 184 silicone elastomer kit)

PGMEA developer

Silicon Wafers

Silver pellets

Silver wire

SU-8 2001

TNT T7 Quick Coupled Transcription/Translation System

Tris

Titanium
RS Components, Germany

LifeTechnologies, Paisley, UK

Olympus Corp, Tokyo, JP

Avanti Polar Lipids, Alabaster, USA

Sigma Aldrich, Taufkirchen, Germany

Edelmetall - Handel GmbH \& Co.KG Rheinstetten, Germany

Präzisionsglas \& Optik GmbH, Isernloh, Germany

Sigma Aldrich, Taufkirchen, Germany

Sigma Aldrich, Taufkirchen, Germany

Sigma Aldrich, Taufkirchen, Germany

LifeTechnologies, Paisley, UK

Dow Corning, Wiesbaden, Germany

MicroChem, Newton, US

Si-Mat, Kaufering, Germany

MaTeck, Jülich, Germany

Goodfellow, Bad Nauheim, Germany

MicroChem, Newton, US

Promega, Madison, US

Sigma Aldrich, Taufkirchen, Germany

MaTeck, Jülich, Germany 


\section{Equipment \& software}

Atomic Force Microscope

E-Beam \& thermal evaporation

Fluorescence Microscope

Impedance Spectrometer

LIH8+8 DA/AD converter

Mask Aligner

Patch Clamp Amplifier

Patchmaster

pCLAMP/Clampfit

RIE/PECVD Cluster

Scanning Elektron Microscope

Versa Studio

ZView
Cypher, Asylum Research, Santa Barbara, US

Univex350, OerlikonLeybold Vacuum, Köln, Germany

Zeiss,

VersaStat4, Princton Applied Research, Oak Ridge, US

HEKA - Elektronik, Lambrecht, Germany

MJB4,Karl SÜSS - MicroTec AG, Garching, Germany

ELC 03 XS, NPI - Electronics, Tamm, Germany

HEKA - Elektronik, Lambrecht, Germany

Axon Instruments, Union City, US

Plasmalab Systems 100, Oxford Instruments, Bristol, UK)

Philips SEM 515

Princton Applied Research, Oak Ridge, US

(Scribner Associates,Inc, Southern Pines, US 



\section{Danksagung}

An erster Stelle möchte ich mich bei meinem Betreuer, Prof. Christoph Schmidt, bedanken, der mir diese interessante Dissertation ermöglicht hat. Mein anfänglich geäußerter Wunsch, mich in neue biophysikalische Methoden einarbeiten zu können, wurde mit diesem Projekt ermöglicht. Des Weiteren wusste ich die Freiheit und Selbständigkeit, die mir in der Forschung zugestanden wurden, ebenso wie die zahlreichen Möglichkeiten meine Arbeit zu präsentieren, sehr zu schätzen.

Prof. Claudia Steinem danke ich für die Übernahme des Koreferats und ihre Bereitschaft, Zeit für Fragen und Diskussionen in Sachen Impedanzspektroskopie und Einzelkanalmessungen zu finden, und für die Bereitstellung des CPEO3 für die Funktionalisierung der Substrate.

Ohne Jochen Herbst und seine immense Geduld bei der Wartung und Reparatur der Reinraumausstattung auch in Notfällen weit nach Feierabend, sowie zahlreicher Tipps und Tricks in Mikrofabrikationsfragen, wäre dieses Projekt nicht möglich gewesen.

Der gleiche Dank gebührt Jörg Malindretos, der für jedes Problem bezüglich des Mask-Aligners und des Mikroskops immer ansprechbar war.

Clemens Trinks und Thorsten Gronemann danke ich für die Einführung in KOHNassätzverfahren und den Umgang mit der Wafersäge.

Matthias Hahn und Lennart Quintscher danke ich für die SEM Aufnahmen.

Frédéric Eghiaian, Sylvain Monnier und Sebastian Köhler danke ich für die Hilfe im Umgang mit dem Cypher AFM.

Conrad Weichbrodt danke ich für die Einführung in die Impedanzspektroskopie und für die ersten Messungen meiner Substrate. Oliver Gaßmann hat mir die experimentelle Herangehensweise der Einzelkanalmessung nähergebracht, Ole Schütte hat mich bei deren Auswertung unterstützt.

Bei Prof. Sarah Köster, Martha Brennich und Bernd Nöding bedanke ich mich 
für die Hilfe bei dem Design der Photomasken und dem Umgang mit der CADSoftware sowie die hilfreichen Diskussionen über Soft-Lithographie.

Ich danke Kerstin von Roden, Ulrike Schulz und Charlotte Willms sowie Dieter Klopfenstein, die mich in Praxis und Theorie bei allen biochemischen Verfahren unterstützt haben.

Bei Dr. Karl Lautscham und den Mitarbeitern in der Elektronikwerkstatt möchte ich mich für die Hilfe beim Thema Erdung und Abschirmung bedanken. Jedes pA zählt.

Dieter Hille und seinen Mitarbeitern in der Feinmechanikwerkstatt danke ich für die Anfertigung diverser Probenkammern, Masken und Halterungen, Beratung bei Materialfragen und Inventor-Software Problemen.

Thomas Geiling möchte ich für die Unterstützung in EDV-Fragen danken.

Dem SFB803 und dem GGNB danke ich für die Möglichkeiten zur Weiterbildung in diversen Kursen sowie für die zahlreichen Winterschulen und Retreats.

Dem Arbeitskreis danke ich für die schöne Zeit in der Physik Göttingen und auf den diversen DPG Tagungen.

Meinen Korrekturleser/innen danke ich für das schnelle und sorgfältige Lesen der Arbeit, wie auch für die Lektionen in englischer Grammatik.

Die Arbeit wurde finanziell unterstützt durch den SFB803, den GGNB und die Deutsche Forschungsgemeinschaft.

Schlussendlich gilt der größte Dank meinen Eltern, Schwestern und Schwiegereltern, meinen Freunden (Galina, Fritz, Malte, Florian, Doodle, Gunter, Hauke, Phi, Jette und Rainer) und besonders meinem Ehemann Uli dafür, dass sie sowohl die großartigen Dinge als auch die dunklen Zeiten während dieser vier Jahre mit mir geteilt haben. Was wäre ich ohne Euch?

Danke 


\section{Theresa Kaufeld}

Curriculum Vitae

\section{Education}

2004 Abitur, Marion-Dönhoff-Gymnasium, Nienburg/Weser.

2004-2009 Study of Chemistry, Georg-August University Göttingen.

2009 Diploma Thesis, Title: Der Einfluss von Shiga Toxin auf die laterale Mobilität von festkörperunterstützten Lipidmembranen, Supervisor: Prof. Claudia Steinem, Georg-August University Göttingen.

2009-2013 PhD Thesis, Title: Lab-on-chip design to characterize pore-spanning lipid bilayers, Supervisor: Prof. Christoph F. Schmidt, Georg-August University Göttingen.

\section{Talks \& posters}

2010 Poster, DPG Spring Meeting of the Condensed Matter Section, Regensburg, Microarray device for local electrical recording of planar lipid bilayers.

2011 Poster, DPG Spring Meeting of the Condensed Matter Section, Dresden, Porespanning lipid bilayers on microchips.

2012 Talk, March 2012 Meeting of the American Physical Society, Boston, MA, USA Microporous device for local electric recordings on lipid bilayers .

2012 Talk, DPG Spring Meeting of the Condensed Matter Section, Berlin, Local electric recordings of lipid bilayers supported on a microfabricated microporous device.

2013 Poster, DPG Spring Meeting of the Condensed Matter Section, Regensburg, Porespanning lipid bilayers on microchips. 